\title{
Description and Analysis of the Second Spectrum of Tantalum, Ta II
}

\author{
C. C. Kiess
}

(October 23, 1961)

\begin{abstract}
The spectrum has been observed from 2000.73 to 7813.40 A. There are 2,629 lines listed, of which 1,890 have been classified. Observed Zeeman patterns are recorded, as well as $g$-values. These have aided greatly in the work on analysis. The 61 known even levels have been grouped into 26 terms from the $5 d^{3} 6 s, 5 d^{2} 6 s^{2}$ and $5 d^{4}$ configurations. The odd levels number 164; they have no assigned term designations. No series have been found, but the ionization potential is estimated to be between 16.0 and 16.5 electron volts.
\end{abstract}

\section{Introduction}

During the period 1930-1940 the concave-grating and quartz-prism spectrographs of the National Bureau of Standards were used to record photographically the spectra of several elements throughout the region from $1950 \mathrm{~A}$ in the ultraviolet to approximataly $13000 \mathrm{~A}$ in the infrared. The purpose of the investigations was to acquire data for descriptions of the spectra sufficiently extensive and homogeneous to serve for the analysis of their termstructures. These descriptions as finally tabulated comprise the wavelengths of the spectral lines, their estimated intensities, and their Zeeman effects. Thus far results have been published for the first and second spectra of chromium, Cr I and Cr II, [1], ${ }^{1}$ and for the second spectrum of molybdenum, Mo II [2]. This paper presents similar results for the second spectrum of tantalum, Ta II. A subsequent paper will present results for Ta I. It is of interest to note that no detailed description of this spectrum has appeared since the publication of volume 6 of Kayser's Handbuch der Spectroscopie in 1912 [3], although the M.I.T. Tables of Wavelengths [4] do contain new wavelengths and estimated intensities for some of the stronger lines of Ta II.

\section{Experimental Procedures}

In the spectroscopy laboratory of this Bureau it was a common practice, whenever a spectrograph was adjusted for a given spectral region, to photograph the spectra of several elements during a period of observation. Thus, tantalum was one of the elements investigated concurrently with chromium and molybdenum. It is, therefore, unnecessary to repeat the statements, in the earlier papers, concerning the instruments, techniques, and accessories employed in making the observations. The electrodes used in the light-sources for this investigation were tantalum rods of high purity procured from the Fansteel Co. The only impurities detected in them, from measurements of the spectrograms, were a small amount of niobium (columbium), and a possible trace of wolfram (tungsten).

\footnotetext{
${ }^{1}$ Figures in brackets indicate the literature references at the end of this paper.
}

The first observations of the Zeeman effect for this Bureau's work on the spectra of tantalum were made for us by B. E. Moore who used the electromagnet and grating-spectrograph of the Brace Laboratory of the University of Nebraska. His first results showed well-resolved patterns for many of the stronger lines of both Ta I and Ta II. However, his untimely death in 1925, brought to an end his cooperation in the work. With the acquisition of a Weiss watercooled magnet by the NBS the work was continued here. Our first observations were made with the 21-ft concave grating ruled by Rowland with 20,000 lines per inch. The low reflectance of this instrument in the short-wave regions limited its use to the visible spectrum. Subsequently additional observations were made with the large Hilger E 185 quartzprism spectrograph, and with the grating-spectrograph carrying the concave grating ruled by $\mathrm{R}$. W. Wood with 30,000 lines per inch. Thus observations of the Zeeman effect, at fields of approximately 35,000 oersteds were obtained for lines of Ta I and Ta II in the spectral range from 2180 to $8300 \mathrm{~A}$. Subsequently the excellent plates obtained at M.I.T. under the direction of $G$. R. Harrison were sent to the NBS for measurement and study. All lines from 2300 to 7700 A, showing magnetic broadening or splitting, were measured even though many of them were overlaid by the Zeeman patterns of adjacent lines.

\section{Results}

\subsection{Wavelengths, Intensities, and Zeeman Effects}

The results of this investigation are presented in tables 1, 2, and 3 . The wavelengths listed in the first column of table 1 are, in general, mean values derived from measurements of two or more spectrograms. A few lines, measured only once on a morestrongly exposed plate, have been kept because their reality has been indicated by the term-analysis. Many of the lines exhibit hyperfine structure, which is designated by the letter $c$ after the estimated intensity in the second column. The wavelengths adopted for such lines are weighted means of the 
components measured in the complex patterns. It is recognized that this is not a strictly legitimate procedure because in most of the complex lines the fainter components are not fully resolved; but the adopted wavelength is sufficiently close to the undetermined real value to serve the requirements for identification and classification.

The intensities of the lines are estimated values based on the strength of photographic blackening they produce. The number 1 is assigned to the weakest lines measurable on a spectrogram, the stronger ones being graded on a linear scale relative to the faintest. It is obvious that intensities derived in this manner are not comparable in spectral regions that require different types of photographic emulsions to record them. The lines owe their complexity to a nuclear spin of $7 / 2$, measured by Mc.Millan and Grace [5], for the isotope 181, in which all but 0.01 of the nuclear mass is concentrated.

The wave-numbers of the lines in the third column were taken from Kayser's Tabelle der Schwingungszahlen, but have been corrected by the small amounts necessary for conformity with Edlén's [6] formula for the index of refraction of air. These numbers are the basis for the analysis of the term-structure of Ta II, and are equal to the differences between the energy-levels of the Ta atom involved in the production of the spectral lines. These level-differences are given in column 4 . In the last column are given the Zeeman patterns from either the M.I.T. or the NBS spectrograms. Those given to only two places of decimals are from. the NBS plates; all others from those made at M.I.T. The type of shading in unresolved magnetic patterns, which gives a clue to the $J$ - and $g$-values of the combining levels, are indicated by letters with the significance, $A=1 \mathrm{~N}$, $B=\uparrow \uparrow, C=\wedge, D=\sqcap$.

\subsection{The Systems of Energy Levels}

Analysis of Ta II was begun independently and almost simultaneously at three laboratories. When G. R. Harrison of the M.I.'T. Spectroscopy Laboratory learned that the problem had been undertaken at the Spectroscopy Laboratory of the NBS he suggested that a joint announcement be made of the first results that had been obtained, and that the necessary continuation of the work be carried on at the NBS with the aid of the M.I.T. data. This suggestion was realized in part in the paper, "Preliminary list of terms of Ta II," [7]. At the same time a similar and somewhat more extensive paper by van den Berg [8] was issued by the Zeeman Laboratory of the University of Amsterdam. This earlier work showed that the prominent low even terms of Ta II arise in the electron configurations $5 d^{3} 6 s, 5 d^{2} 6 s^{2}$, and $5 d^{4}$, the clue to their identity having been found in well-resolved Zeeman patterns that are in very close accord with those from levels with Landé $g$-values.

The further procedure of unravelling the spectrum has followed the conventional practices of combing the spectrum for pairs and groups of lines separated by established level-differences, and of tying together related lines that exhibit the same $g$-values. These operations have been somewhat tedious; but as the structure of the spectrum began to unfold the work received guidance from the calculations of Trees et al., [9] which narrowed the limits of search for the levels expected theoretically.

In table 2 are listed the low even terms upon which the spectrum is built. The ground state $a^{5} \mathrm{~F}$ arises in the electron configuration $5 d^{3}\left({ }^{4} \mathrm{~F}\right) 6 s$; but interspersed among its components are levels from the ${ }^{3} \mathrm{~F}$ and ${ }^{3} \mathrm{P}$ terms of $5 d^{2} 6 s^{2}$, which thus becomes a strong competitor of $5 d^{3} 6 s$ in governing the energetic processes of the atom. All of the $5 d^{3} 6 s$ terms have been found; and likewise all but ${ }^{1} \mathrm{~S}$ of $5 d^{2} 6 s^{2}$. The configuration $5 d^{4}$ requires more energy to excite its levels, with the result that only six of its 16 theoretically possible terms have been detected completely or partially. Resolved Zeeman patterns of a small number of unclassified lines indicate that transitions to undetected $5 d^{4}$ levels do occur, but the connecting links have not yet been found.

The $g$-values that are assigned to the levels in table 2 are, for most of them, mean values derived from two to twenty well-resolved Zeeman patterns. They are sufficiently close to $L S$-values to justify the designation of the levels with the $L S$ notation, although a study, by Trees $[9,10,11]$ of the configuration interaction in tantalum indicates that most of the levels of table 2 are of mixed parentage.

The odd levels in table 3 reveal the extent to which the excited states of the ionized atom have departed from LS-coupling. A few of the lowest odd levels might be uniquely designated with LSsymbols, but to apply this mode of designation to the others would be meaningless. It has seemed best, therefore, for sake of brevity, to give as the designation of the line in table 1 the first three figures of the level-value and the appropriate inner quantum number.

\subsection{Series and Ionjzation Potential}

No high levels have been found that can be ascribed unambiguously to the configurations $5 d^{3} n s$ and $5 d^{2} 6 s n s$. It is impossible, therefore, at this stage of the analysis of 'Ta II, to calculate an ionizationpotential from series-forming term.s. An ionization potential of $7.88 \mathrm{ev}$ has been found for the neutral Ta atom; for neutral $W$ this value is $7.98 \mathrm{ev}$. For the homologous atoms $\mathrm{Nb}$ and Mo the estimated second ionization potentials are a little more than twice the first. If this ratio is of physical significance we may expect, therefore, that the ionization potential of singly ionized tantalum lies between 16 and $16.5 \mathrm{ev}$, which is essentially the same as the interpolated value given by Finkelnburg and Humbach [12].

During its progress the work described above has been aided with observational material from various individuals, and consultations with others. As stated previously, the late B. E. Moore, of the University of Nebraska, made the first magnetic observations that were of significance in analyzing the 
spectra Ta I and Ta II. Later G. R. Harrison and W. J. Hitcheock of M.I.T. made available their Zeeman observations and the results which they had obtained from them. At the NBS, R. E. Trees has made numerous theoretical calculations to verify and identify the levels found for the low configurations and to predict the probable positions of others;
R. Zalubas has obtained and measured several spectrograms to improve the description of Ta II in the region 2500 to $1950 \mathrm{~A}$; and Mrs. Ruth Peterson has made extensive calculations and tabulations of the data. It is a pleasure for me to acknowledge my indebtedness to them and also to others who have made contributions to this work.

TABIE 1. Wavelengths, term combinations, and Zeeman effects of Ta II

\begin{tabular}{|c|c|c|c|c|}
\hline $\begin{array}{l}\text { Wave } \\
\text { length } \\
\lambda \text { air }\end{array}$ & $\begin{array}{l}\text { Intensity } \\
\text { and } \\
\text { notes }\end{array}$ & $\begin{array}{l}\text { Wave } \\
\text { number }\end{array}$ & $\begin{array}{c}\text { Term } \\
\text { combination }\end{array}$ & Zeeman pattern \\
\hline $\begin{array}{l}7813.40 \\
7733.92 \\
7606.07 \\
7510.64 \\
6736.86\end{array}$ & $\begin{array}{l}2 \\
2 \\
4 \\
2 \\
1\end{array}$ & $\begin{array}{l}\text { 12795. } 01 \\
12926.05 \\
13143.78 \\
13310.78 \\
14839.61\end{array}$ & $c{ }^{3} \mathrm{P}_{2}-413(1)$ & \\
\hline $\begin{array}{r}11.75 \\
6598.80 \\
97.09 \\
67.94 \\
34.22\end{array}$ & $\begin{array}{l}1 \\
4 \\
5 \\
3 \\
2\end{array}$ & $\begin{array}{l}14895.13 \\
15150.08 \\
15154.03 \\
15221.27 \\
15299.82\end{array}$ & $\begin{array}{l}c{ }^{3} \mathrm{~F}_{3}-385(2) \\
c{ }^{3} \mathrm{P}_{0}-385(1) \\
d^{3} \mathrm{~F}_{4}-468(3)\end{array}$ & $(\mathbf{0 . 0 0}, 0.22,0.46) 0.54 A$ \\
\hline $\begin{array}{r}00.81 \\
6496.20 \\
05.76 \\
6374.86 \\
69.04\end{array}$ & $\begin{array}{r}5 \\
1 \\
3 \\
10 \\
18\end{array}$ & $\begin{array}{l}15378.45 \\
15389.36 \\
15606.64 \\
15682.38 \\
15696.61\end{array}$ & $\begin{array}{l}b^{3} \mathrm{G}_{5}-466(4) \\
c^{3} \mathrm{~F}_{2}-385(1) \\
a^{1} \mathrm{D}_{2}-292(2)\end{array}$ & $\begin{array}{ll}(\mathbf{0 . 0 0}, 0.27) & \mathbf{1 . 2 1}, 1.44 \\
(0.59, \mathbf{1 . 1 9}) & 0.00, \mathbf{0 . 5 2}, \mathbf{1 . 1 0}, 1.72\end{array}$ \\
\hline $\begin{array}{r}11.50 \\
6286.85 \\
80.22 \\
73.54 \\
39.91\end{array}$ & $\begin{array}{l}1 \\
1 \\
4 \\
1 \\
1\end{array}$ & $\begin{array}{l}15839.71 \\
15901.82 \\
15918.61 \\
15935.55 \\
16021.44\end{array}$ & $\begin{array}{l}b^{3} \mathrm{G}_{4}-440(4) \\
c{ }^{3} \mathrm{P}_{1}-421(2) \\
c^{1} \mathrm{D}_{2}-489(3) \\
b^{3} \mathrm{D}_{3}-466(4)\end{array}$ & \\
\hline $\begin{array}{r}6187.75 \\
61.00 \\
57.50 \\
46.46 \\
34.82\end{array}$ & $\begin{array}{r}3 \\
2 \\
5 \\
3 \\
10\end{array}$ & $\begin{array}{l}16156.49 \\
16226.64 \\
16235.86 \\
16265.03 \\
16295.89\end{array}$ & $\begin{array}{l}b{ }^{3} \mathrm{G}_{3}-430(2) \\
b{ }^{3} \mathrm{G}_{4}-444(3)\end{array}$ & $(0.00) 1.10$ \\
\hline $\begin{array}{r}22.58 \\
10.37 \\
6079.18 \\
42.47 \\
17.71\end{array}$ & $\begin{array}{l}3 \\
1 \\
3 \\
1 \\
2\end{array}$ & $\begin{array}{l}\text { 16328. } 46 \\
16361.09 \\
16445.03 \\
16544.94 \\
16613.02\end{array}$ & $\begin{array}{l}c{ }^{1} \mathrm{G}_{4}-514(4) \\
b{ }^{3} \mathrm{H}_{5}-417(4) \\
b^{3}{ }^{3} \mathrm{D}_{2}-468(2) \\
b^{3}{ }^{3} \mathrm{D}_{3}-471(3) \\
c{ }^{3} \mathrm{~F}_{3}-402(2)\end{array}$ & \\
\hline $\begin{array}{r}5989.34 \\
80.86 \\
79.61 \\
72.96 \\
63.72\end{array}$ & $\begin{array}{l}1 \\
5 \\
1 \\
1 \\
1\end{array}$ & $\begin{array}{l}\text { 16691. } 70 \\
16715.37 \\
16718.87 \\
16737.48 \\
16763.41\end{array}$ & $\begin{array}{l}b{ }^{3} \mathrm{G}_{3}-435(3) \\
b{ }^{1} \mathrm{G}_{4}-421(4) \\
b{ }^{3} \mathrm{D}_{2}-471(3)\end{array}$ & \\
\hline $\begin{array}{r}53.85 \\
19.99 \\
5891.18 \\
78.31 \\
62.27\end{array}$ & $\begin{array}{l}1 \\
1 \\
1 \\
7 \\
1\end{array}$ & $\begin{array}{l}16791.20 \\
16887.24 \\
16969.83 \\
17006.98 \\
17053.51\end{array}$ & $\begin{array}{l}c{ }^{3} \mathrm{P}_{2}-448(3) \\
b{ }^{3} \mathrm{D}_{1}-468(2) \\
c{ }^{1} \mathrm{G}_{4}-521(3) \\
d{ }^{3} \mathrm{~F}_{2}-468(2)\end{array}$ & \\
\hline $\begin{array}{r}38.98 \\
33.85 \\
5798.32 \\
94.35 \\
90.78\end{array}$ & $\begin{array}{l}3 \\
1 \\
2 \\
1 \\
2\end{array}$ & $\begin{array}{l}17121.50 \\
17136.59 \\
17241.59 \\
17253.41 \\
17264.04\end{array}$ & $\begin{array}{l}b^{3} \mathrm{H}_{4}-415(3) \\
a^{1} \mathrm{~F}_{3}-421(4)\end{array}$ & \\
\hline $\begin{array}{l}\text { 86. } 72 \\
82.90 \\
53.43 \\
51.78 \\
38.10\end{array}$ & $\begin{array}{r}1 \\
1 \\
3 \\
15 \\
1\end{array}$ & $\begin{array}{l}17276.17 \\
17287.57 \\
17376.12 \\
17381.10 \\
17422.54\end{array}$ & $\begin{array}{l}b{ }^{3} \mathrm{H}_{4}-417(5) \\
c c^{1} \mathrm{D}_{2}-503(3) \\
c{ }^{3} \mathrm{~F}_{2}-403(1) \\
a^{5} \mathrm{P}_{2}-292(2)\end{array}$ & $(0.91, \mathbf{1 . 8 0}) \ldots, \mathbf{0 . 5 2}, \mathbf{1 . 4 0}, 2.34$ \\
\hline
\end{tabular}


TABLE 1. Wavelengths, term combinations, and Zeeman effects of Ta II-Continued

\begin{tabular}{|c|c|c|c|c|}
\hline $\begin{array}{l}\text { Wave } \\
\text { length } \\
\text { 入air }\end{array}$ & $\begin{array}{l}\text { Intensity } \\
\text { and } \\
\text { notes }\end{array}$ & $\begin{array}{l}\text { Wave } \\
\text { number }\end{array}$ & $\begin{array}{c}\text { Term } \\
\text { combination }\end{array}$ & Zeeman pattern \\
\hline $\begin{array}{r}5735.53 \\
35.21 \\
30.44 \\
18.33 \\
16.07\end{array}$ & $\begin{array}{l}8 \\
2 \\
4 \\
8 \\
3\end{array}$ & $\begin{array}{l}17430.34 \\
17431.32 \\
17445.83 \\
17482.77 \\
17489.69\end{array}$ & $\begin{array}{l}b{ }^{3} \mathrm{G}_{3}-442(2) \\
d^{3} \mathrm{~F}_{4}-489(3) \\
a^{1} \mathrm{H}_{5}-417(5) \\
a^{3} \mathrm{G}_{3}-292(2)\end{array}$ & $(\mathbf{0 . 0 0}, 0.39) \mathbf{0 . 0 0} w, 0.46,0.85$ \\
\hline $\begin{array}{r}04.72 \\
5688.62 \\
60.32 \\
57.40 \\
49.61\end{array}$ & $\begin{array}{r}12 \\
1 \\
1 \\
3 \\
1\end{array}$ & $\begin{array}{l}17524.48 \\
17574.08 \\
17661.95 \\
17671.06 \\
17695.43\end{array}$ & $\begin{array}{c}c{ }^{3} \mathrm{~F}_{3}-411(2) \\
b{ }^{1} \mathrm{G}_{4}-429(3) \\
d^{3} \mathrm{~F}_{2}-475(2)\end{array}$ & $(0.000) 0.964$ \\
\hline $\begin{array}{l}37.82 \\
36.43 \\
35.95 \\
\text { 20. } 19 \\
04.46\end{array}$ & $\begin{array}{l}1 \\
8 \\
8 \\
2 \\
1\end{array}$ & $\begin{array}{l}17732.43 \\
17736.81 \\
17738.32 \\
17788.06 \\
17837.98\end{array}$ & $\begin{array}{l}a^{5} \mathrm{D}_{1}-312(1) \\
b^{3} \mathrm{G}_{5}-490(5) \\
b^{3} \mathrm{D}_{1}-478(0)\end{array}$ & \\
\hline $\begin{array}{r}00.63 \\
5576.61 \\
74.46 \\
72.20 \\
69.80\end{array}$ & $\begin{array}{r}18 \\
1 \\
15 \\
1 \\
10\end{array}$ & $\begin{array}{l}17850.18 \\
17927.06 \\
17933.98 \\
17941.25 \\
17948.98\end{array}$ & $\begin{array}{l}b{ }^{1} \mathrm{D}_{2}-411(2) \\
a^{3} \mathrm{H}_{5}-361(4) \\
c^{3} \mathrm{~F}_{3}-415(3) \\
a^{1} \mathrm{P}_{1}-413(1)\end{array}$ & \\
\hline $\begin{array}{l}62.18 \\
57.32 \\
52.64 \\
52.10 \\
51.65\end{array}$ & $\begin{array}{l}4 \\
4 \\
8 \\
5 \\
4\end{array}$ & $\begin{array}{l}\text { 17973. } 57 \\
17989.29 \\
18004.46 \\
18006.20 \\
18007.66\end{array}$ & $\begin{array}{l}c{ }^{3} \mathrm{P}_{0}-413(1) \\
a^{5} \mathrm{D}_{3}-337(2) \\
d^{3} \mathrm{~F}_{4}-495(3) \\
b^{3} \mathrm{G}_{3}-448(3)\end{array}$ & \\
\hline $\begin{array}{r}51.27 \\
05.39 \\
5494.42 \\
92.94 \\
71.74\end{array}$ & $\begin{array}{l}3 \\
7 \\
7 \\
2 \\
5\end{array}$ & $\begin{array}{l}18008.90 \\
18158.97 \\
18195.23 \\
18200.13 \\
18270.65\end{array}$ & $\begin{array}{ll}b & { }^{1} \mathrm{G}_{4}-435(3) \\
a & { }^{1} \mathrm{~F}_{3}-430(2) \\
c & { }^{3} \mathrm{P}_{1}-444(0)\end{array}$ & \\
\hline $\begin{array}{l}63.22 \\
51.98 \\
41.98 \\
39.40 \\
16.62\end{array}$ & $\begin{array}{r}8 \\
3 \\
3 \\
10 \\
1\end{array}$ & $\begin{array}{l}\text { 18299. } 14 \\
18336.87 \\
18370.56 \\
18379.27 \\
18456.57\end{array}$ & $\begin{array}{l}c{ }^{1} \mathrm{D}_{2}-513(1) \\
b^{3} \mathrm{D}_{2}-487(1) \\
d^{3} \mathrm{~F}_{2}-482(2) \\
b^{3} \mathrm{D}_{3}-490(2)\end{array}$ & \\
\hline $\begin{array}{r}09.67 \\
5378.90 \\
77.45 \\
73.62 \\
69.73\end{array}$ & $\begin{array}{l}6 \\
4 \\
3 \\
2 \\
5\end{array}$ & $\begin{array}{l}\text { 18480. } 28 \\
\text { 18585. } 99 \\
18591.01 \\
18604.26 \\
18617.73\end{array}$ & $\begin{array}{l}b{ }^{3} \mathrm{G}_{4}-466(4) \\
b^{3} \mathrm{H}_{5}-440(4) \\
b^{3} \mathrm{G}_{3}-454(2)\end{array}$ & \\
\hline $\begin{array}{l}69.19 \\
67.44 \\
55.82 \\
51.10 \\
44.57\end{array}$ & $\begin{array}{l}4 \\
4 \\
7 \\
2 \\
8\end{array}$ & $\begin{array}{l}\text { 18619. } 64 \\
18625.66 \\
18666.09 \\
18682.55 \\
18705.38\end{array}$ & $\left\{\begin{array}{l}b^{3} \mathrm{G}_{5}-498(5) \\
c^{3} \mathrm{~F}_{2}-415(3) \\
c^{3} \mathrm{~F}_{4}-417(5) \\
b^{3} \mathrm{G}_{4}-468(3)\end{array}\right.$ & \\
\hline $\begin{array}{l}37.24 \\
32.66 \\
27.06 \\
21.15 \\
20.69\end{array}$ & $\begin{array}{l}1 \\
1 \\
1 \\
7 \\
2\end{array}$ & $\begin{array}{l}18731.07 \\
18747.15 \\
18766.86 \\
18787.71 \\
18789.33\end{array}$ & $a^{1} \mathrm{P}_{1}-421(2)$ & \\
\hline $\begin{array}{r}18.66 \\
06.67 \\
01.53 \\
01.16 \\
5292.44\end{array}$ & $\begin{array}{l}7 \\
1 \\
2 \\
3 \\
2\end{array}$ & $\begin{array}{l}\text { 18796. } 50 \\
18838.97 \\
18857.23 \\
18858.55 \\
18889.62\end{array}$ & $b^{1} \mathrm{D}_{2}-421(2)$ & \\
\hline $\begin{array}{l}90.32 \\
86.15 \\
66.48 \\
60.67 \\
55.47\end{array}$ & $\begin{array}{r}7 \\
2 \\
7 \\
25 \\
4\end{array}$ & $\begin{array}{l}\text { 18897. } 19 \\
18912.10 \\
18982.72 \\
19003.70 \\
19022.48\end{array}$ & $\begin{array}{l}c{ }^{1} \mathrm{G}_{4}-540(5) \\
b{ }^{3} \mathrm{D}_{3}-495(3) \\
\\
b^{3}{ }^{3} \mathrm{G}_{4}-471(3) \\
\left.b^{3}\right)_{3}-496(3)\end{array}$ & $\begin{array}{l}(0.00 W) 0.93 A \\
(0.000 W) 0.999 \\
(0.59) 1.16\end{array}$ \\
\hline
\end{tabular}


TABLE 1. Wavelengths, term combinations, and Zeeman effects of Ta $\mathrm{II}$-Continued

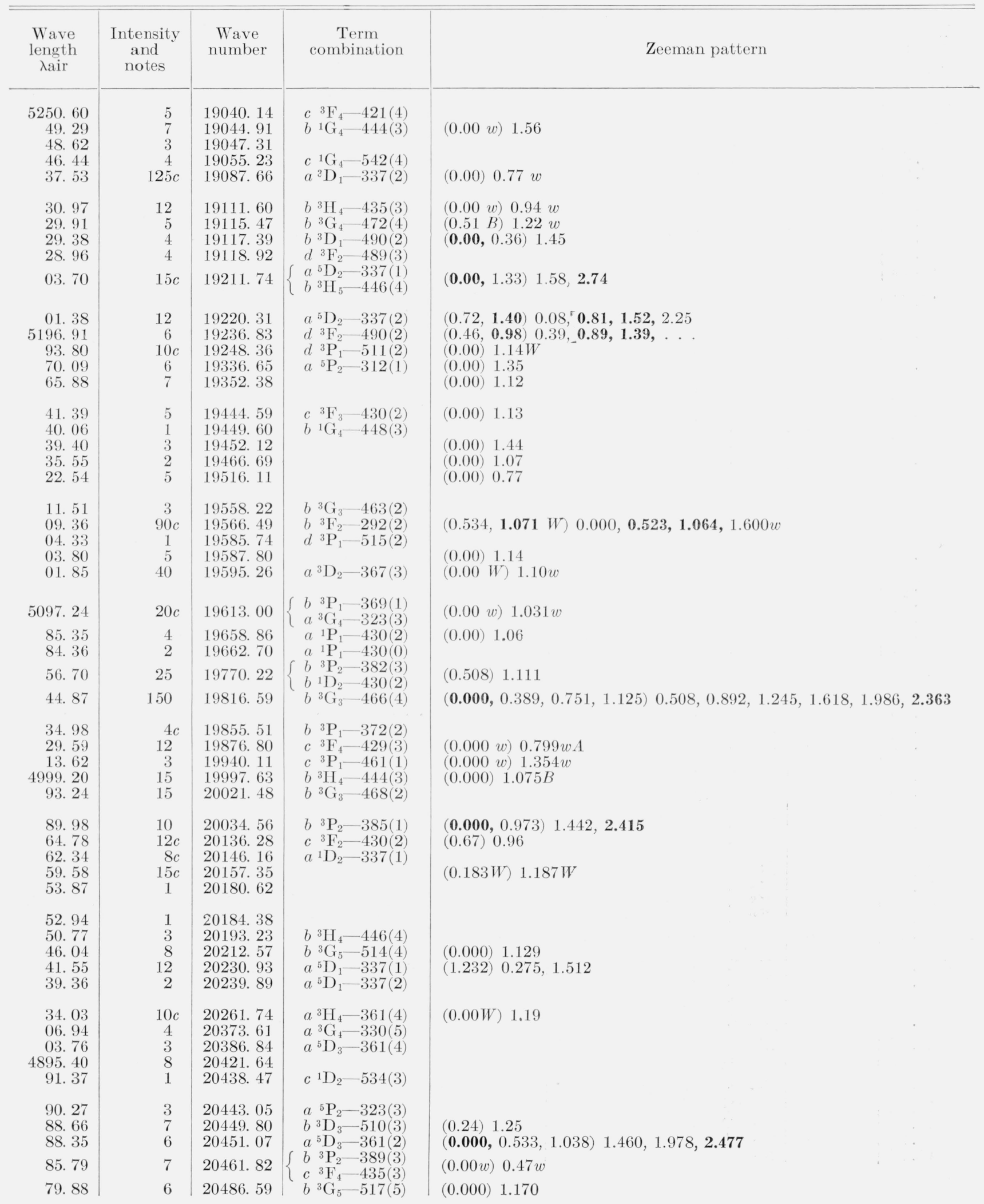


TABLE 1. Wavelengths, term combinations, and Zeeman effects of Ta $\mathrm{II}$-Continued

\begin{tabular}{|c|c|c|c|c|}
\hline $\begin{array}{l}\text { Wave } \\
\text { length } \\
\quad \text { 入air }\end{array}$ & $\begin{array}{l}\text { Intensity } \\
\text { and } \\
\text { notes }\end{array}$ & $\begin{array}{l}\text { Wave } \\
\text { number }\end{array}$ & $\begin{array}{c}\text { Term } \\
\text { combination }\end{array}$ & Zeeman pattern \\
\hline $\begin{array}{r}4876.40 \\
73.19 \\
66.79 \\
64.48 \\
61.03\end{array}$ & $\begin{array}{c}2 \\
3 \\
2 \\
50 c \\
3\end{array}$ & $\begin{array}{l}20501.18 \\
20514.70 \\
20541.70 \\
20551.42 \\
20566.03\end{array}$ & $\begin{array}{l}c{ }^{3} \mathrm{P}_{1}-467(2) \\
a^{3} \mathrm{G}_{3}-323(3)\end{array}$ & $\begin{array}{l}(0.00) 1.07 \\
(0.00 W) 0.93 w \\
(0.000) 0.986\end{array}$ \\
\hline $\begin{array}{l}60.51 \\
59.29 \\
58.38 \\
55.51 \\
43.84\end{array}$ & $\begin{array}{l}3 \\
2 \\
7 \\
8 \\
5\end{array}$ & $\begin{array}{l}\text { 20568. } 24 \\
20573.38 \\
20577.27 \\
20589.42 \\
20639.00\end{array}$ & $\begin{array}{l}b^{3} \mathrm{D}_{1}-505(1) \\
b^{3} \mathrm{D}_{3}-511(2) \\
a \\
{ }^{1} \mathrm{~F}_{3}-454(2) \\
d^{3} \mathrm{~F}_{4}-521(3) \\
c^{3} \mathrm{~F}_{3}-442(2)\end{array}$ & $\begin{array}{l}(0.00) 1.12 \\
(\mathbf{0 . 0 0 0}, 0.189,0.372) \mathbf{0 . 6 1 3}, 0.783,0.959, \ldots \\
(0.000 W) 1.114 w \\
(\mathbf{0 . 0 0 0}, 0.174,0.351) \\
\mathbf{0 . 7 1 9}, 0.906,1.070, \ldots .\end{array}$ \\
\hline $\begin{array}{l}38.54 \\
37.00 \\
32.95 \\
32.50 \\
29.67\end{array}$ & $\begin{array}{r}1 \\
2 \\
1 \\
1 \\
10\end{array}$ & $\begin{array}{l}\text { 20661. } 60 \\
20668.20 \\
20685.50 \\
20687.43 \\
20699.56\end{array}$ & $\begin{array}{l}b{ }^{3} \mathrm{D}_{2}-510(3) \\
b{ }^{3} \mathrm{G}_{3}-475(2) \\
d{ }^{3} \mathrm{~F}_{2}-505(1) \\
b{ }^{3} \mathrm{P}_{0}-369(1)\end{array}$ & $(0.000) 0.686 w$ \\
\hline $\begin{array}{l}24.98 \\
08.26 \\
06.37 \\
05.87 \\
04.03\end{array}$ & $\begin{array}{r}4 \\
6 \\
3 \\
12 \\
10\end{array}$ & $\begin{array}{l}\text { 20719. } 70 \\
20791.72 \\
20799.92 \\
20802.09 \\
20810.03\end{array}$ & $\begin{array}{cc}c & { }^{1} \mathrm{D}_{2}-537(1) \\
b & { }^{3} \mathrm{D}_{2}-511(2) \\
a & { }^{1} \mathrm{P}_{1}-442(1) \\
b & { }^{3} \mathrm{~F}_{4}-392(3) \\
c & { }^{3} \mathrm{~F}_{3}-444(3)\end{array}$ & $(0.129,0.254, \mathbf{0 . 3 9 0}) 0.683,0.819, \mathbf{0 . 9 3 9}, \mathbf{1 . 0 6 5}, 1.213,1.333$ \\
\hline $\begin{array}{r}4798.57 \\
94.09 \\
93.28 \\
89.43 \\
85.68\end{array}$ & $\begin{array}{r}2 \\
15 \\
3 \\
4 \\
4\end{array}$ & $\begin{array}{l}\text { 20833. } 72 \\
20853.18 \\
20856.73 \\
20873.47 \\
20889.82\end{array}$ & $\begin{array}{l}a{ }^{1} \mathrm{P}_{1}-442(2) \\
b^{3} \mathrm{H}_{5}-462(4) \\
b^{3} \mathrm{G}_{4}-490(5)\end{array}$ & $\begin{array}{l}(0.000) 1.302 w \\
(0.00) 1.57\end{array}$ \\
\hline $\begin{array}{l}\text { 78. } 25 \\
65.18 \\
64.71 \\
61.42 \\
60.79\end{array}$ & $\begin{array}{l}8 \\
4 \\
4 \\
7 \\
5\end{array}$ & $\begin{array}{l}\text { 20922. } 33 \\
20979.70 \\
20981.77 \\
20996.24 \\
20999.04\end{array}$ & $\begin{array}{l}c{ }^{3} \mathrm{~F}_{4}-440(4) \\
b^{3} \mathrm{G}_{3}-478(3)\end{array}$ & $\begin{array}{l}(0.360,0.698, \mathbf{1 . 0 3 6}) 0.488, \mathbf{0 . 8 4 4}, \mathbf{1 . 2 0 0}, 1.552,1.913 \\
(0.000 w) 1.252 w\end{array}$ \\
\hline $\begin{array}{l}59.31 \\
52.36 \\
51.96 \\
36.75 \\
31.27\end{array}$ & $\begin{array}{r}2 \\
3 \\
7 \\
35 \\
8\end{array}$ & $\begin{array}{l}21005.59 \\
21036.76 \\
21038.03 \\
21105.60 \\
21130.05\end{array}$ & $\begin{array}{l}c{ }^{3} \mathrm{~F}_{3}-446(4) \\
c{ }^{3} \mathrm{P}_{2}-490(2) \\
a^{5} \mathrm{D}_{3}-367(3) \\
a^{5} \mathrm{D}_{0}-337(1)\end{array}$ & $\begin{array}{l}(0.559, \mathbf{0 . 9 3 7}) \ldots ., 1.159, \mathbf{1 . 4 5 8}, 1.754 \\
(0.000) 0.270 \\
(0.000 w) 0.704 w\end{array}$ \\
\hline $\begin{array}{l}28.96 \\
26.84 \\
24.54 \\
17.93 \\
15.28\end{array}$ & $\begin{array}{l}5 \\
5 c \\
3 \\
8 \\
7 c\end{array}$ & $\begin{array}{l}21140.39 \\
21149.89 \\
21160.15 \\
21189.80 \\
21201.71\end{array}$ & $\begin{array}{l}b{ }^{3} \mathrm{P}_{1}-385(2) \\
b \quad{ }^{3} \mathrm{P}_{1}-385(1) \\
a^{3} \mathrm{D}_{3}-397(4)\end{array}$ & $(0.000) 0.996 B$ \\
\hline $\begin{array}{l}\text { 12. } 37 \\
11.79 \\
08.66 \\
08.07 \\
04.54\end{array}$ & $\begin{array}{r}2 \\
1 \\
10 \\
1 \\
3\end{array}$ & $\begin{array}{l}21214.83 \\
21217.42 \\
21231.52 \\
21234.17 \\
21250.12\end{array}$ & $\begin{array}{l}c{ }^{3} \mathrm{~F}_{3}-448(3) \\
b{ }^{3} \mathrm{H}_{5}-466(4) \\
b^{3} \mathrm{D}_{1}-511(2) \\
b{ }^{3} \mathrm{~F}_{4}-397(4)\end{array}$ & $\begin{array}{l}(\mathbf{0 . 0 0 0}, 0.152,0.315,0.465,0.620) \mathbf{0 . 4 2 5}, 0.588,0.744,0.898,1.056,1.208 \\
(0.000 W) 1.231 w\end{array}$ \\
\hline $\begin{array}{r}\text { 4698. } 46 \\
\text { 86. } 79 \\
84.04\end{array}$ & $\begin{array}{l}3 \\
5 \\
3\end{array}$ & $\begin{array}{l}21277,63 \\
21330,60 \\
21343,10\end{array}$ & $\begin{array}{l}c{ }^{3} \mathrm{~F}_{2}-442(1) \\
c{ }^{3} \mathrm{~F}_{2}-442(2)\end{array}$ & $(\mathbf{0 . 0 0 0}, 0.442) 0.699, \mathbf{1 . 1 5 8} w$ \\
\hline $\begin{array}{l}\text { 83. } 07 \\
79.64\end{array}$ & $\begin{array}{r}20 \\
3\end{array}$ & $\begin{array}{l}21347,54 \\
21363.20\end{array}$ & $\left\{\begin{array}{l}a^{3} \mathrm{D}_{2}-385(2) \\
c{ }^{3} \mathrm{~F}_{4}-444(3) \\
b^{3} \mathrm{D}_{1}-513(1)\end{array}\right.$ & $\begin{array}{l}(0.235, \mathbf{0 . 4 0 2}) \quad 0.864, \mathbf{1 . 0 4 0 , 1 . 2 3 2 ,} 1.404 \\
(0.057) 1.017\end{array}$ \\
\hline 78.01 & 10 & 21370.64 & $\left\{\begin{array}{l}c{ }^{1} \mathrm{G}_{4}-565(5) \\
b^{3} \mathrm{G}_{4}-495(3)\end{array}\right.$ & $(0.00 \mathrm{~W}) 1.47 \mathrm{~B}$ \\
\hline $\begin{array}{l}\text { 74. } 93 \\
\text { 70. } 78 \\
\text { 67. } 24 \\
\text { 65. } 94\end{array}$ & $\begin{array}{l}2 \\
2 \\
4 \\
1\end{array}$ & $\begin{array}{l}21384.70 \\
21403.72 \\
21419,96 \\
21425.89\end{array}$ & & \\
\hline
\end{tabular}


TABLE 1. Wavelengths, term combinations, and Zeeman effects of Ta II-Continued

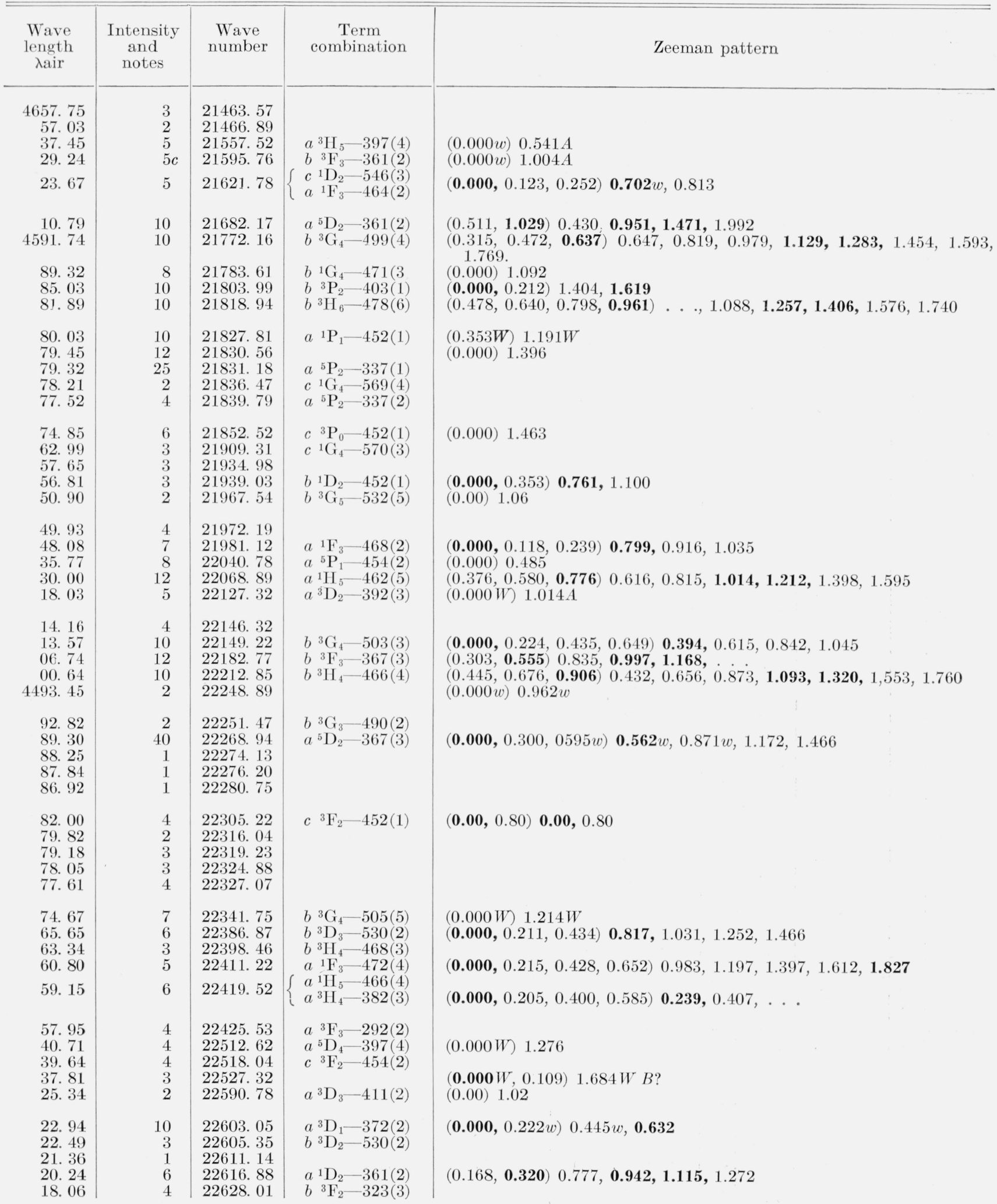


TABLE 1. Wavelengths, term combinations, and Zeeman effects of Ta II-Continued

\begin{tabular}{|c|c|c|c|c|}
\hline $\begin{array}{l}\text { Wave } \\
\text { length } \\
\quad \lambda \text { air }\end{array}$ & $\begin{array}{l}\text { Intensity } \\
\text { and } \\
\text { notes }\end{array}$ & $\begin{array}{l}\text { Wave } \\
\text { number }\end{array}$ & $\begin{array}{l}\text { Term } \\
\text { combination }\end{array}$ & Zeeman pattern \\
\hline 4413. 89 & 8 & 22649. 39 & $\left\{\begin{array}{l}a^{3} \mathrm{H}_{5}-408(6) \\
b^{3} \mathrm{~F}_{3}-372(2)\end{array}\right.$ & \\
\hline 08.92 & 3 & 22674. 90 & $d^{3} \mathrm{~F}_{4}-542(4)$ & $(0.000) 1.160 w$ \\
\hline 03. 73 & 25 & 22701.66 & $a^{5} \mathrm{D}_{1}-361(2)$ & $(\mathbf{0 . 0 0 0}, 0.562) \quad \mathbf{0 . 3 9 9}, 0.950,1.509$ \\
\hline 01. 13 & 4 & $\begin{array}{l}22715.06 \\
22719.24\end{array}$ & & 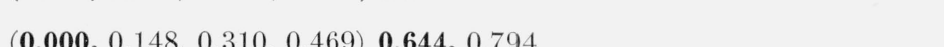 \\
\hline & & & & \\
\hline 4398. 85 & 3 & 22726.84 & & \\
\hline 97.06 & 8 & 22736. 09 & $\left\{\begin{array}{l}a^{5} \mathrm{D}_{2}-372(2) \\
b^{3} \mathrm{H}_{4}-471(3)\end{array}\right.$ & $(\mathbf{0 . 0 0 0}, 0.113,0.240, \ldots) 0.842 w A$ \\
\hline & 2 & & $b^{3} \mathrm{G}_{3}-495(2)$ & \\
\hline $\begin{array}{l}90.84 \\
86.77\end{array}$ & $\begin{array}{r}12 \\
4\end{array}$ & $\begin{array}{l}22768.28 \\
22789.43\end{array}$ & $\begin{array}{l}a{ }^{1} \mathrm{P}_{1}-461(1) \\
a^{5} \mathrm{D}_{3}-385(2)\end{array}$ & $\begin{array}{l}(0.000 w) 0.852 w \\
(\mathbf{0 . 0 0 0 ,} 0.459) 1.455,1.876,2.309\end{array}$ \\
\hline $\begin{array}{l}81.39 \\
76.75 \\
74.47 \\
74.21 \\
73.06\end{array}$ & $\begin{array}{r}8 \\
5 \\
20 \\
50 \\
3\end{array}$ & $\begin{array}{l}22817.40 \\
22841.60 \\
22853.50 \\
22854.83 \\
22860.84\end{array}$ & $\begin{array}{l}b^{3} \mathrm{G}_{3}-476(3) \\
b^{3} \mathrm{D}_{3}-534(3) \\
a^{3} \mathrm{H}_{6}-408(6) \\
b{ }^{3} \mathrm{P}_{2}-413(1) \\
b^{3} \mathrm{D}_{1}-528(1)\end{array}$ & $\begin{array}{l}(0.198,0.379, \mathbf{0 . 5 6 0}) 0.478,0.665, \mathbf{0 . 8 4 9}, \mathbf{1 . 0 3 4}, 1.222,1.390 \\
(0.069) 1.208 \\
(0.434) 1.120 \\
(\mathbf{0 . 0 0 0 ,} 0.432) \mathbf{1 . 0 0 7}, 1.328\end{array}$ \\
\hline $\begin{array}{l}\text { 71. } 48 \\
71.11 \\
63.96 \\
62.51 \\
60.02\end{array}$ & $\begin{array}{l}1 \\
2 \\
8 \\
2 \\
4\end{array}$ & $\begin{array}{l}22869.10 \\
22871.08 \\
22908.51 \\
22916.13 \\
22929.24\end{array}$ & $\begin{array}{l}c{ }^{3} \mathrm{~F}_{3}-464(2) \\
b{ }^{3} \mathrm{G}_{4}-510(3) \\
b{ }^{3} \mathrm{P}_{1}-403(1)\end{array}$ & $\begin{array}{l}(\mathbf{0 . 0 0 0}, 0.101,0.206,0.314) \mathbf{0 . 7 8 1}, 0.848,0.959 \\
(0.000 w) 1.282 B\end{array}$ \\
\hline $\begin{array}{l}\text { 58. } 14 \\
\text { 56. } 42 \\
\text { 54. } 27\end{array}$ & $\begin{array}{r}40 \\
2 \\
3\end{array}$ & $\begin{array}{l}\text { 22939. } 13 \\
\text { 22948. } 16 \\
\text { 22959. } 50\end{array}$ & $b^{3} \mathrm{G}_{5}-542(4)$ & $(0.000) 1.126$ \\
\hline 50. 21 & 8 & 22980. 94 & $\left\{\begin{array}{l}d^{3}{ }^{3} \mathrm{~F}_{2}-528(1) \\
a^{1} \mathrm{P}_{1}-463(2)\end{array}\right.$ & $(0.00) 1.48$ \\
\hline 47. 88 & 15 & 22993. 24 & $a^{5} \mathrm{P}_{1}-337(1)$ & \\
\hline $\begin{array}{l}46.53 \\
41.85 \\
41.00 \\
38.24 \\
37.61\end{array}$ & $\begin{array}{r}10 \\
2 \\
8 \\
12 \\
4\end{array}$ & $\begin{array}{l}\text { 23000. } 39 \\
23025.17 \\
23029.68 \\
23044.36 \\
23047.69\end{array}$ & $\begin{array}{l}a^{3} \mathrm{D}_{3}-415(3) \\
c \\
{ }^{3} \mathrm{~F}_{3}-466(4) \\
c \\
{ }^{3} \mathrm{P}_{2}-510(3) \\
b^{3} \mathrm{H}_{6}-490(5) \\
b^{3} \mathrm{D}_{1}-530(2)\end{array}$ & 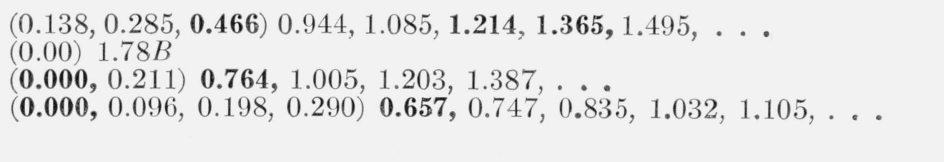 \\
\hline $\begin{array}{l}\text { 35. } 21 \\
\text { 31. } 15 \\
\text { 28. } 79 \\
\text { 27. } 58 \\
\text { 26. } 21\end{array}$ & $\begin{array}{l}2 \\
6 \\
7 \\
5 \\
3\end{array}$ & $\begin{array}{l}\text { 23060. } 44 \\
\text { 23082. } 07 \\
\text { 23094. } 67 \\
\text { 23101. } 13 \\
\text { 23108. } 42\end{array}$ & $b{ }^{3} \mathrm{~F}_{4}-415(3)$ & \\
\hline $\begin{array}{l}\text { 24. } 65 \\
\text { 21. } 05 \\
\text { 20. } 38 \\
\text { 17. } 86 \\
\text { 11. } 12\end{array}$ & $\begin{array}{l}5 \\
4 \\
2 \\
2 \\
2\end{array}$ & $\begin{array}{l}23116.77 \\
23136.04 \\
23139.62 \\
23153.09 \\
23189.33\end{array}$ & $\begin{array}{l}d{ }^{3} \mathrm{~F}_{4}-546(3) \\
a^{3} \mathrm{D}_{2}-403(1) \\
c{ }^{3} \mathrm{P}_{2}-511(2)\end{array}$ & $\begin{array}{l}(0.000) 1.228 w \\
(0.000) 1.319\end{array}$ \\
\hline $\begin{array}{l}09.77 \\
07.11 \\
06.83 \\
05.28 \\
03.52\end{array}$ & $\begin{array}{r}1 \\
4 \\
15 \\
3 \\
15\end{array}$ & $\begin{array}{l}23196.58 \\
23210.88 \\
23212.43 \\
23220.77 \\
23230.25\end{array}$ & $\begin{array}{ll}b & { }^{1} \mathrm{D}_{2}-464(2) \\
c & { }^{3} \mathrm{~F}_{3}-468(3) \\
c & { }^{3} \mathrm{~F}_{4}-462(5) \\
c & \\
{ }^{3} \mathrm{~F}_{3}-468(2)\end{array}$ & $\begin{array}{l}(\mathbf{0 . 0 0 0}, 0.170,0.334,0.516) 0.842,1.018,1.199,1.366,1.548,1.722,1.913 \\
(0.000) 1.054\end{array}$ \\
\hline $\begin{array}{r}4294.02 \\
82.01 \\
80.85 \\
79.96 \\
75.69\end{array}$ & $\begin{array}{r}12 \\
8 \\
3 \\
2 \\
2\end{array}$ & $\begin{array}{l}23281.65 \\
23346.96 \\
23353.28 \\
23358.13 \\
23381.45\end{array}$ & $\begin{array}{ll}b & { }^{3} \mathrm{~F}_{4}-417(4) \\
a & { }^{1} \mathrm{~F}_{3}-482(2) \\
c & { }^{3} \mathrm{P}_{1}-495(2)\end{array}$ & $\begin{array}{ll}(0.000) & 1.250 \\
(0.000) & 0.996 \\
(0.00) & 0.59\end{array}$ \\
\hline $\begin{array}{l}\text { 73. } 68 \\
71.09 \\
67.30 \\
65.05 \\
64.43\end{array}$ & $\begin{array}{r}3 \\
7 \\
30 \\
2 \\
1\end{array}$ & $\begin{array}{l}23392.48 \\
23406.62 \\
23427.45 \\
23439.80 \\
23443.18\end{array}$ & $\begin{array}{l}b{ }^{3} \mathrm{H}_{4}-478(3) \\
a{ }^{1} \mathrm{D}_{2}-369(1)\end{array}$ & $\begin{array}{l}(0.00) 0.34 A \\
(\mathbf{0 . 0 0}, 0.35) 0.63,1.18, \mathbf{1 . 5 9}\end{array}$ \\
\hline
\end{tabular}


TABLE 1. Wavelengths, term combinations, and Zeeman effects of Ta II-Continued

\begin{tabular}{|c|c|c|c|c|}
\hline $\begin{array}{l}\text { Wave } \\
\text { length } \\
\lambda \text { air }\end{array}$ & $\begin{array}{c}\text { Intensity } \\
\text { and } \\
\text { notes }\end{array}$ & $\begin{array}{l}\text { Wave } \\
\text { number }\end{array}$ & $\begin{array}{l}\text { Term } \\
\text { combination }\end{array}$ & Zeeman pattern \\
\hline 4263.50 & 3 & 23448. 32 & & \\
\hline 62.95 & 4 & 23451.32 & & $(0.00) \quad 1.26$ \\
\hline 61. 63 & 4 & 23458.59 & $c{ }^{3} \mathrm{~F}_{2}-463(2)$ & \\
\hline 55. 92 & 2 & 23490.04 & ${ }^{c}{ }^{3} \mathrm{P}_{2}-515(2)$ & $(0.00) 1.41$ \\
\hline 42. 74 & 20 & 23563. 02 & $\begin{cases}c & { }^{3} \mathrm{~F}_{2}-464(2) \\
c & { }^{3} \mathrm{~F}_{4}-466(4)\end{cases}$ & $(0.357,0.546, \mathbf{0 . 7 2 1}) 0.667,0.874, \mathbf{1 . 0 3 2}, \mathbf{1 . 2 1 4}, 1.385,1.562,1.745$ \\
\hline 41. 59 & 8 & 23569. 15 & $a^{3} \mathrm{D}_{3}-421(4)$ & $(0.000) 1.132$ \\
\hline 35. 93 & 15 & 23600.91 & $a^{1} \mathrm{~F}_{3}-484(4)$ & $(\mathbf{0 . 0 0 0}, 0.286,0.572,0.859) 0.996,1.272,1.554,1.826, \mathbf{2 . 1 1 5}$ \\
\hline 35.51 & 7 & 23603. 26 & $\left\{\begin{array}{l}d^{3} \mathrm{P}_{1}-555(2) \\
a^{1} \mathrm{H}_{5}-478(6)\end{array}\right.$ & $(0.00) 1.42$ \\
\hline 30.87 & 40 & 23629. 16 & $b^{3} \mathrm{~F}_{4}-421(4)$ & $(0.000 W) 1.248$ \\
\hline 23. 49 & 6 & 23670.44 & $a^{1} \mathrm{D}_{2}-372(2)$ & $(0.48, \mathbf{0 . 9 8}) 0.11, \mathbf{0 . 6 3}, \mathbf{1 . 1 6}, 1.65$ \\
\hline 20. 09 & 3 & 23689.53 & $b^{3} \mathrm{~F}_{3}-382(3)$ & \\
\hline 19. 70 & 1 & 23691. 71 & & $(0.00) 1.06$ \\
\hline 18. 44 & 12 & 23698. 79 & & $(0.102) 1.186$ \\
\hline 13. 43 & 4 & 23726. 93 & $a^{3} \mathrm{H}_{6}-417(5)$ & $(0.00 \mathrm{~W}) 0.92$ \\
\hline 13. 02 & 2 & 23729.27 & & $(0.16) 0.85$ \\
\hline 09.57 & 6 & 23748. 71 & $c{ }^{3} \mathrm{~F}_{4}-468(3)$ & $(\mathbf{0 . 0 0}, 0.16,0.36,0.53) \mathbf{0 . 5 0}, 0.69, \ldots$ \\
\hline 08. 42 & 12 & 23755. 20 & $a^{5} \mathrm{D}_{1}-372(2)$ & $(\mathbf{0 . 0 0 0}, 0.892)-\mathbf{0 . 2 6 7} w,+0.611,1.493$ \\
\hline 01.02 & $15 c$ & 23797.06 & $a^{1} \mathrm{~F}_{3}-486(2)$ & $(0.00 w) 0.81 w$ \\
\hline 00.30 & 2 & 23801. 10 & $d^{3} \mathrm{~F}_{2}-536(2)$ & $(0.00) 1.19$ \\
\hline 4190.51 & 10 & 23856. 70 & & $(0.000 w) \mathbf{1 . 2 4 4}, 1.738$ \\
\hline 87. 43 & 10 & 23874. 27 & $b^{1} \mathrm{D}_{2}-471(3)$ & $(0.000) 1.076$ \\
\hline 85. 06 & 7 & 23887. 81 & $a^{3} \mathrm{D}_{1}-385(2)$ & $(\mathbf{0 . 0 0 0}, 0.169) \mathbf{1 . 0 8 0}, 1.234$ \\
\hline 83. 93 & 7 & 23894. 22 & $c{ }^{3} \mathrm{~F}_{3}-475(2)$ & $(\mathbf{0 . 0 0 0}, 0.220,0.428) \mathbf{0 . 6 3 6}, 0.815$ \\
\hline 82. 43 & 8 & 23902.80 & $c{ }^{3} \mathrm{~F}_{2}-468(3)$ & $(\mathbf{0 . 0 0}, 0.50,1.03) 1.13,1.65,2.18$ \\
\hline 81. 64 & $8 c$ & 23907.35 & $a^{3} \mathrm{D}_{1}-385(1)$ & $(0.00) 1.20$ \\
\hline 79. 06 & $15 c$ & 23922. 07 & $c{ }^{3} \mathrm{~F}_{2}-468(2)$ & $(0.392, \mathbf{0 . 7 9 2} w) \mathbf{1 . 0 9 8 , 1 . 4 9 7 w}$ \\
\hline 76. 93 & $25 c$ & 23934. 30 & $b^{3} \mathrm{~F}_{3}-385(2)$ & $(0.000) 0.988$ \\
\hline 76. 62 & 1 & 23936. 09 & $a^{3} \mathrm{H}_{5}-421(4)$ & $(0.00) 0.98 B$ \\
\hline 69. 03 & $15 c$ & 23979. 62 & $b{ }^{3} \mathrm{P}_{1}-413(1)$ & \\
\hline & & 24016. 28 & $b{ }^{3} \mathrm{~F}_{2}-337(1)$ & \\
\hline 62.45 & $8 c$ & 24017.54 & $a^{5} \mathrm{D}_{3}-397(4)$ & \\
\hline 61. 20 & $12 c$ & 24024. 77 & $\left\{\begin{array}{l}b{ }^{3} \mathrm{D}_{3}-546(3) \\
b{ }^{3} \mathrm{~F}_{2}-337(2)\end{array}\right.$ & $(0.282, \mathbf{0 . 5 3 2}) \ldots ?, 0.536, \mathbf{0 . 8 0 7 , \mathbf { 1 . 0 6 1 }}$ \\
\hline 59. 02 & 3 & 24037.34 & $b^{3} \mathrm{H}_{4}-484(4)$ & \\
\hline 58.51 & 3 & 24040.31 & $a^{5} \mathrm{D}_{2}-385(1)$ & \\
\hline 55. 40 & $7 c$ & 24058. 29 & $a^{3} \mathrm{G}_{4}-367(3)$ & $(0.162 W) 0.754 W$ \\
\hline 52. 19 & 5 & 24076. 89 & $c{ }^{3} \mathrm{P}_{2}-521(3)$ & \\
\hline 39. 52 & 7 & 24150.58 & $b^{1}{ }^{1} \mathrm{G}_{4}-495(3)$ & $(\mathbf{0 . 0 0 0}, 0.220,0.432,0.652) \mathbf{0 . 4 3 8}, 0.657,0.868,1.090,1.310$ \\
\hline 33. 35 & 5 & 24186.65 & $a^{3} \mathrm{D}_{2}-413(1)$ & $(0.00,0.71) 0.55,1.15$ \\
\hline 30. 22 & 4 & 24204. 95 & $c{ }^{3} \mathrm{~F}_{3}-478(3)$ & $(0.261,0.382) 1.18$ \\
\hline 24. 65 & 8 & 24237.67 & $b^{3} \mathrm{G}_{5}-555(5)$ & $(0.000 \mathrm{~W}) 0.871 \mathrm{~A}$ \\
\hline 20. 67 & 8 & 24261.03 & $b^{1} \mathrm{G}_{4}-496(3)$ & $(0.000) 1.196 w$ \\
\hline 10. 10 & 5 & 24323. 44 & $a^{5} \mathrm{D}_{4}-415(3)$ & $(0.000) 1.183 B$ \\
\hline 09.40 & 4 & 24327.57 & $\left\{\begin{array}{l}b{ }^{3} \mathrm{G}_{4}-524(4) \\
a^{5} \mathrm{P}_{3}-367(3)\end{array}\right.$ & $(0.121) 1.062$ \\
\hline 4099. 42 & $8 c$ & 24386. 81 & $a^{5} \mathrm{D}_{0}-369(1)$ & $(0.000) 0.682 w$ \\
\hline 95.53 & $8 c$ & 24409. 97 & $a^{3} \mathrm{G}_{3}-361(2)$ & $(0.000) 0.913 B$ \\
\hline 94. 60 & 3 & 24415.52 & & $(\mathbf{0 . 0 0}, 0.36,0.75,1.13) \mathbf{0 . 7 3}, 1.10, \ldots$ \\
\hline 86. 17 & $2 c$ & 24465.88 & $b^{3} \mathrm{~F}_{4}-429(3)$ & $(0.000) 0.797 \mathrm{w}$ \\
\hline 85. 07 & 1 & 24472.48 & $b^{3} \mathrm{H}_{5}-498(5)$ & $(0.30, \mathbf{0 . 5 2}) 0.27$ \\
\hline 84. 13 & 2 & 24478. 12 & $a^{5} \mathrm{D}_{4}-417(5)$ & $(0.000) 1.291 A$ \\
\hline 75.50 & 10 & 24529.94 & $\left\{\begin{array}{l}b{ }^{3} \mathrm{H}_{4}-489(3) \\
b{ }^{1} \mathrm{D}_{2}-478(3)\end{array}\right.$ & $(\mathbf{0 . 0 0}, 0.30,0.60,0.91) \mathbf{0 . 0 8}, 0.38,0.68,1.00$ \\
\hline 74. 43 & 1 & 24536. 38 & $c{ }^{3} \mathrm{P}_{2}-525(3)$ & $(0.000) 1.454 w$ \\
\hline 73. 13 & $7 c$ & 24544.20 & $a^{5} \mathrm{D}_{4}-417(4)$ & $(0.00 \mathrm{~W}) 1.19 \mathrm{~W}$ \\
\hline 66. 22 & 4 & 24585.95 & $c{ }^{3} \mathrm{~F}_{2}-475(2)$ & $(0.58, \mathbf{1 . 1 9}) 0.00 w, \mathbf{0 . 7 1}, \mathbf{1 . 3 0}, 1.88$ \\
\hline 60. 21 & 4 & 24622.33 & $b^{3} \mathrm{H}_{4}-490(5)$ & $(\mathbf{0 . 0 0 0 , 0 . 2 3 7 , 0 . 4 8 8 , 0 . 7 3 9 , 0 . 9 9 1 ) \ldots , 1 . 7 0 2 , 1 . 9 5 0 , 2 . 1 9 5}$ \\
\hline 54. 15 & 2 & 24659. 14 & & \\
\hline
\end{tabular}


TABLE 1. Wavelengths, term combinations, and Zeeman effects of Ta II-Continued

\begin{tabular}{|c|c|c|c|c|}
\hline $\begin{array}{c}\text { Wave } \\
\text { length } \\
\text { 入air }\end{array}$ & $\begin{array}{l}\text { Intensity } \\
\text { and } \\
\text { notes }\end{array}$ & $\begin{array}{l}\text { Wave } \\
\text { number }\end{array}$ & $\begin{array}{c}\text { Term } \\
\text { combination }\end{array}$ & Zeeman pattern \\
\hline 4050.00 & 3 & 24684. 41 & & (0.073) 1.062 \\
\hline 49. 09 & 1 & 24689. 91 & $d^{3} \mathrm{~F}_{2}-545(2)$ & \\
\hline 48. 31 & 2 & 24694. 70 & & $(\mathbf{0 . 0 0 0}, 0.291,0.580) 1.149,1.426, \mathbf{1 . 7 1 2}$ \\
\hline $\begin{array}{l}45.02 \\
40.47\end{array}$ & $\begin{array}{l}3 c \\
4 c\end{array}$ & $\begin{array}{l}24714.76 \\
24742.61\end{array}$ & $\begin{array}{ll}b & { }^{3} \mathrm{~F}_{2}-392(3) \\
c & { }^{3} \mathrm{~F}_{4}-478(3)\end{array}$ & $(\mathbf{0 . 0 0 0}, 0.168,0.344,0.522) \mathbf{0 . 4 9 7}, 0.671,0.846,1.020$ \\
\hline & $2 c$ & 2477825 & h $3 \mathrm{P}$ & $(0.00) 123$ \\
\hline $\begin{array}{l}\text { 34. } 66 \\
30.96\end{array}$ & $\begin{array}{r}2 c \\
10 c\end{array}$ & $\begin{array}{l}2468.25 \\
24801.00\end{array}$ & $a^{5} \mathrm{D}_{2}-392(3)$ & $\begin{array}{l}(0.00) 1.23 \\
(\mathbf{0 . 0 0 0}, 0.336,0.662) \mathbf{0 . 4 7 0} w, 0.804,1.137,1.470\end{array}$ \\
\hline 29. 08 & 2 & 24812.58 & & $(\mathbf{0 . 0 0 0}, 0.427,0.845) \mathbf{0 . 0 7 7}, 0.548,0.964, \ldots$ \\
\hline 16. 82 & 5 & 24888. 27 & a ${ }^{5} \mathrm{P}_{2}-367(3)$ & $(\mathbf{0 . 0 0 0}, 0.252,0.502) \mathbf{0 . 6 6 6}, 0.914,1.162, \ldots$ \\
\hline 16. 28 & 7 & 24891. 64 & $a^{5} \mathrm{D}_{4}-421(4)$ & $(0.193 W A) 1.234 W$ \\
\hline 10. 49 & 3 & 24927.55 & $\left\{\begin{array}{lll}b^{3}{ }^{3} \mathrm{D}_{3}-555(2) & \\
b^{1} \mathrm{D}_{2}-482(2)\end{array}\right.$ & \\
\hline 10. 24 & 5 & 24929. 08 & $b^{1} \mathrm{G}_{4}-503(3)$ & $(\mathbf{0 . 0 0 0}, 0.224,0.451,0.663) \mathbf{0 . 6 4 0}, 0.849,1.052$ \\
\hline 05.97 & 5 & 24955. 69 & & $(\mathbf{0 . 0 0 0}, 0.269,0.518) \mathbf{0 . 6 9 6}, 0.950,1.212, \ldots$ \\
\hline 04.83 & 3 & 24962. 77 & $c{ }^{3} \mathrm{P}_{1}-511(2)$ & $(0.000 w) 1.347 w$ \\
\hline 3999. 39 & $3 c$ & 24996. 74 & $a^{3} \mathrm{G}_{3}-367(3)$ & $(0.358, \mathbf{0 . 8 8 0}) 0.811, \mathbf{1 . 3 1 4}, \mathbf{1 . 8 1 5}, 2.332$ \\
\hline 92. 44 & 10 & 25040. 26 & $a^{5} \mathrm{D}_{1}-385(2)$ & $(\mathbf{0 . 0 0 0 ,} 0.489) \mathbf{0 . 5 1 3}, 1.007,1.497$ \\
\hline 92. 15 & 1 & 25042.05 & $c{ }^{1} \mathrm{D}_{2}-580(1)$ & $(0.000) 0.850$ \\
\hline 90. 76 & 10 & 25050.83 & $b^{3} \mathrm{~F}_{4}-435(3)$ & $(\mathbf{0 . 0 0 0}, 0.158,0.329,0.484) 0.952,1.083,1.229,1.370, \mathbf{1 . 5 0 4}$ \\
\hline 89. 32 & 2 & 25059. 81 & $a^{5} \mathrm{D}_{1}-385(1)$ & (1.04) $0.50,1.39$ \\
\hline 87. 96 & $\overline{3}$ & 25068. 41 & $a^{1} \mathrm{~F}_{3}-499(4)$ & $(\mathbf{0 . 0 0 0}, 0.260,0.505) 1.073,1.356,1.578, \mathbf{1 . 8 3 0}$ \\
\hline 87. 82 & 2 & 25069. 29 & $b^{3} \mathrm{G}_{4}-532(5)$ & \\
\hline 86. 21 & 3 & 25079. 37 & & $(0.000) 1.023$ \\
\hline 84.58 & 2 & 25089.65 & $\left\{\begin{array}{l}b{ }^{3} \mathrm{H}_{4}-495(5) \\
c{ }^{1} \mathrm{G}_{4}-602(3)\end{array}\right.$ & $(0.00 w D) 0.80 A$ \\
\hline 84. 26 & 12 & 25091. 68 & $c^{3} \mathrm{P}_{1}-513(1)$ & $(0.623) 1.046,1.336$ \\
\hline 80. 99 & $25 c$ & 25112. 28 & a ${ }^{5} \mathrm{P}_{2}-369(1)$ & $(\mathbf{0 . 0 0 0}, 0.868) 0.680,1.418,2.151$ \\
\hline 75. 64 & 8 & 25146. 06 & $b^{3} \mathrm{D}_{2}-555(2)$ & $(0.000) 1.049$ \\
\hline 74. 19 & 6 & 25155. 22 & & $(0.496) 1.225 W$ \\
\hline 72. 99 & $18 c$ & 25162.84 & $b^{3} \mathrm{~F}_{3}-397(4)$ & $\begin{array}{l}(\mathbf{0 . 0 0 0}, 0.217,0.442 w 0.781 w) \\
\quad \mathbf{1 . 8 7 5} w\end{array}$ \\
\hline 70.57 & 10 & 25178. 16 & $b^{3} \mathrm{G}_{4}-533(4)$ & $(0.148) 1.099 \mathrm{~W}$ \\
\hline 69. 70 & 2 & 25183. 72 & $b^{3} \mathrm{G}_{5}-564(5)$ & \\
\hline 64. 96 & 4 & 25213. 80 & $b^{3} \mathrm{H}_{4}-496(3)$ & $(0.000) 0.890$ \\
\hline 61. 60 & 20 & 25235. 18 & $b^{3} \mathrm{D}_{3}-558(2)$ & \\
\hline 58.55 & 3 & 25254.63 & $b^{3} \mathrm{G}_{5}-565(5)$ & $(0.00 W) 0.58$ \\
\hline 57. 62 & 4 & 25260.58 & $a^{1} \mathrm{P}_{1}-486(2)$ & $(0.000) 1.130$ \\
\hline & & 25268. 66 & & \\
\hline 54. 68 & 5 & 25279. 37 & $b^{3} \mathrm{H}_{5}-506(6)$ & \\
\hline 52. 70 & 7 & 25292.04 & $b^{3} \mathrm{G}_{3}-521(3)$ & $(0.478, \mathbf{0 . 7 3 8}) 0.358,0.593, \mathbf{0 . 8 4 1}, \mathbf{1 . 0 9 4}, 1.329$ \\
\hline 51. 50 & $10 c$ & 25299.67 & $c{ }^{3} \mathrm{P}_{1}-515(2)$ & $(\mathbf{0 . 0 0 0}, 0.113) 1.196, \mathbf{1 . 3 1 0}$ \\
\hline 44. 85 & 10 & 25342. 31 & $c{ }^{3} \mathrm{~F}_{3}-489(3)$ & $(0.195,0.394, \mathbf{0 . 6 0 2}) \quad 0.675,0.874, \mathbf{1 . 0 7 1}, \mathbf{1 . 2 7 1}, 1.462,1.674$ \\
\hline 43. 78 & 10 & 25349. 23 & & $0.000) 0.867$ \\
\hline 42. 84 & 5 & 25355. 26 & $a^{5} \mathrm{P}_{2}-372(2)$ & $(0.84, \mathbf{1 . 5 8}) 0.16, \mathbf{0 . 6 4}, \ldots$ \\
\hline 41. 86 & 3 & 25361.52 & & $(\mathbf{0 . 0 0}, 0.22,0.42) 0.89 A$ \\
\hline 41. 65 & 3 & 25362. 91 & & \\
\hline 40.52 & 4 & 25370.20 & $a^{1} \mathrm{P}_{1}-487(1)$ & $(0.44) 0.75,1.37$ \\
\hline 40. 26 & 6 & 25371.86 & $b{ }^{1} \mathrm{D}_{2}-486(2)$ & $(0.069) 1.133$ \\
\hline 36. 68 & 10 & 25394. 90 & $c{ }^{3} \mathrm{P}_{0}-487(1)$ & (0.000) 1.818 \\
\hline 35. 90 & 3 & 25399. 93 & & \\
\hline 35.57 & 12 & 25402. 11 & $a^{1} \mathrm{D}_{2}-389(3)$ & $(\mathbf{0 . 0 0 0 ,} 0.122,0.230) \mathbf{0 . 7 6 3}, 0.874,1.007,1.142$ \\
\hline $\begin{array}{l}\text { 32. } 85 \\
27.92\end{array}$ & $\begin{array}{l}1 \\
5\end{array}$ & $\begin{array}{l}25419.66 \\
25451.56\end{array}$ & $a^{3} \mathrm{D}_{3}-440(4)$ & $(\mathbf{0 . 0 0 0}, 0.843) 1.600$ \\
\hline 26. 48 & 7 & 25460.90 & & \\
\hline 26. 02 & $12 c$ & 25463.88 & $\left\{\begin{array}{l}a^{3} \mathrm{G}_{3}-372(2) \\
a^{5} \mathrm{P}-361(2)\end{array}\right.$ & $(0.00) 0.85 w$ \\
\hline 24. 56 & $5 c$ & 25473. 38 & $b^{3} \mathrm{D}_{2}-558(1)$ & (0.000) 1.066 \\
\hline $\begin{array}{l}24.15 \\
22.44\end{array}$ & $\begin{array}{c}2 \\
60 c\end{array}$ & $\begin{array}{l}25476.00 \\
25487.09\end{array}$ & $a^{3} \mathrm{~F}_{3}-323(3)$ & $(0.226, \mathbf{0 . 3 5 9}) \quad 0.817, \mathbf{0 . 9 4 0 , 1 . 0 7 4 , 1 . 2 1 4}$ \\
\hline
\end{tabular}


TABLE 1. Wavelengths, term combinations, and Zeeman effects of Ta II-Continued

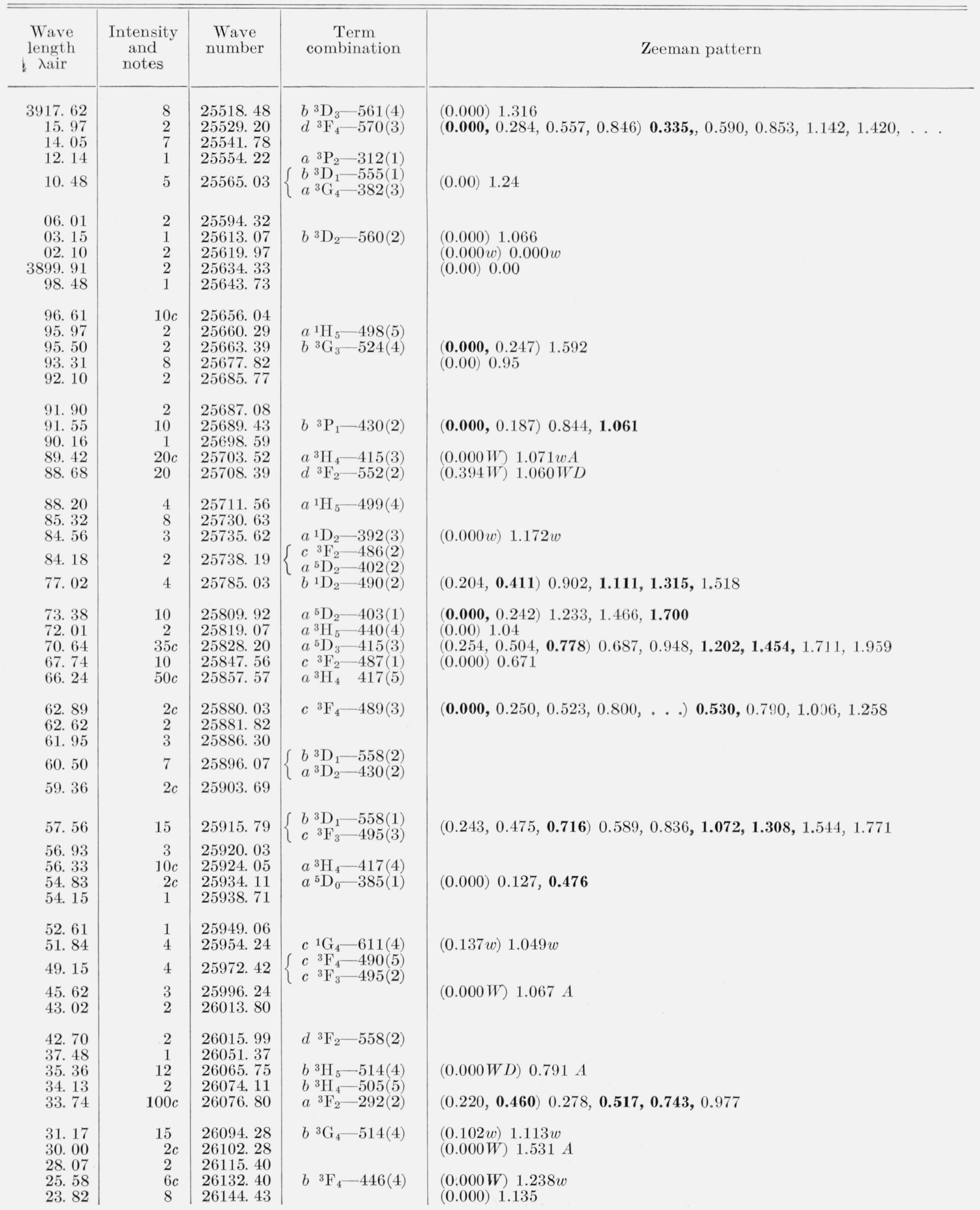


TABLE 1. Wavelengths, term combinations, and Zeeman effects of Ta II-Continued

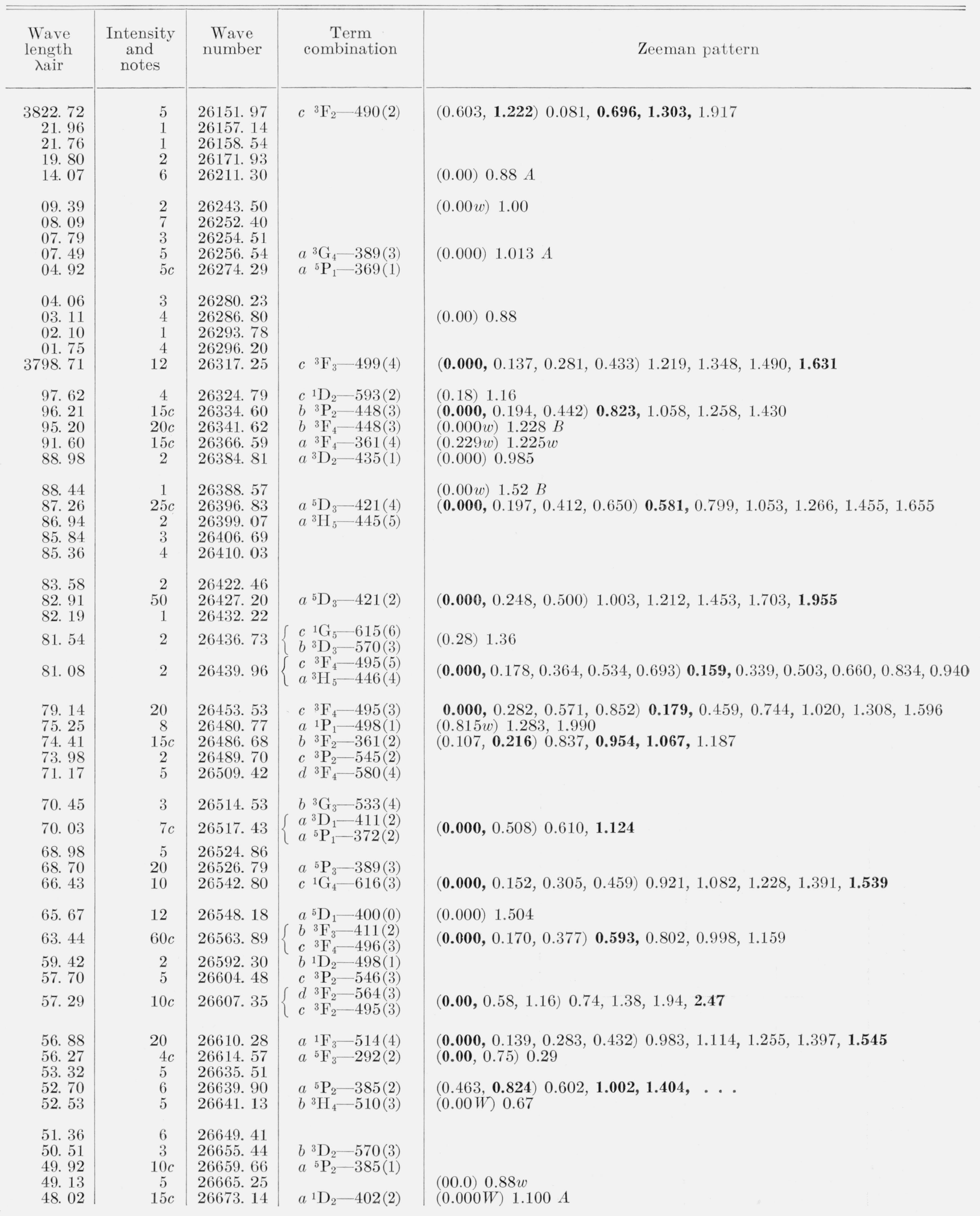


TABLE 1. Wavelengths, term combinations, and Zeeman effects of Ta II-Continued

\begin{tabular}{|c|c|c|c|c|}
\hline $\begin{array}{l}\text { Wave } \\
\text { length } \\
\text { xair }\end{array}$ & $\begin{array}{l}\text { Intensity } \\
\text { and } \\
\text { notes }\end{array}$ & $\begin{array}{l}\text { Wave } \\
\text { number }\end{array}$ & $\begin{array}{l}\text { Term } \\
\text { combination }\end{array}$ & Zeeman pattern \\
\hline 3746.00 & 2 & 26687.56 & & \\
\hline 45.07 & 3 & 26694. 16 & $c^{3} \mathrm{~F}_{3}-503(3)$ & $(0.196,0.414, \mathbf{0 . 6 1 3}) \ldots ?$ \\
\hline 43. 80 & 1 & 26703. 27 & & \\
\hline 40.55 & 4 & 26726.47 & & \\
\hline 37. 50 & $15 c$ & 26748. 22 & $a^{3} \mathrm{G}_{3}-385(2)$ & $(0.000 W) 0.798 w$ \\
\hline 33. 95 & 4 & 26773. 68 & $\left\{\begin{array}{l}b^{3} \mathrm{G}_{5}-580(4) \\
a^{5} \mathrm{D}-440(4)\end{array}\right.$ & $(0.292) 1.169 w$ \\
\hline 33. 41 & 8 & 26777.57 & $\left(\begin{array}{ccc}a & \mathrm{D}_{4}-4 & +40( \pm)\end{array}\right.$ & $\begin{array}{l}(0.000,0.194,0.383,0.560, \mathbf{0 . 7 5 0}) 0.602,0.845,1.033, \mathbf{1 . 2 1 9}, \mathbf{1 . 4 0 4}, \\
\quad 1.588,1.738, \ldots\end{array}$ \\
\hline 21. 94 & $25 c$ & 26860. 10 & $\left\{\begin{array}{l}a{ }^{5} \mathrm{P}_{3}-392(3) \\
a^{5} \mathrm{D}_{2}-413(1)\end{array}\right.$ & $(\mathbf{0 . 0 0 0}, 0.293,0.547) \mathbf{0 . 9 1 7}, 1.169,1.463,1.768,1.976$ \\
\hline 18. 60 & $12 c$ & 26884. 18 & $\begin{cases}a & { }^{3} \mathrm{~F}_{3}-337(2) \\
b & { }^{3} \mathrm{P}_{1}-442(2)\end{cases}$ & $(\mathbf{0 . 0 0 0 ,} 0.309,0.581) 0.047,0.429,0.865,1.176,1.395,1.457, \mathbf{1 . 6 5 1}$ \\
\hline 17. 49 & 4 & 26892. 24 & $a^{5} \mathrm{~F}_{5}-330(5)$ & \\
\hline 07.52 & 6 & 26964.56 & $c{ }^{1} \mathrm{G}_{4}-621(4)$ & $(0.084 w) 1.058 w D$ \\
\hline 00.26 & $20 c$ & 27017. 41 & $a^{3} \mathrm{~F}_{4}-367(3)$ & $(0.000 \mathrm{~W}) 1.324 \mathrm{w}$ \\
\hline 3699. 98 & 8 & 27019.46 & $b^{1} \mathrm{D}_{2}-503(3)$ & $(0.00) 1.16$ \\
\hline 96. 20 & 7 & 27047. 10 & $b{ }^{3} \mathrm{H}_{4}-514(4)$ & $(0.124,0.265,0.411, \mathbf{0 . 5 6 0}) \ldots, \mathbf{0 . 9 9 9 , 1 . 1 4 1 , 1 . 2 6 5 , 1 . 4 0 6 , 1 . 5 3 5}$ \\
\hline 95. 26 & $25 c$ & 27053. 97 & $c{ }^{1} \mathrm{G}_{4}-622(5)$ & \\
\hline 94.50 & $50 c$ & 27059.53 & $\left\{\begin{array}{l}a{ }^{5} \mathrm{D}_{2}-415(3) \\
b{ }^{3} \mathrm{P}_{1}-444(0)\end{array}\right.$ & $(\mathbf{0 . 0 0 0}, 0.266,0.531) \mathbf{0 . 6 7 3}, 0.941,1.201,1.460,1.710$ \\
\hline 92. 62 & $12 c$ & 27073. 32 & $b^{3} \mathrm{~F}_{2}-367(3)$ & $(\mathbf{0 . 0 0 0 , 0 . 1 1 3 , 0 . 2 3 6 )} 1.174,1.286, \mathbf{1 . 4 0 4}$ \\
\hline 91. 85 & 25 & 27078.98 & $b^{3} \mathrm{H}_{5}-524(4)$ & $(0.000) 0.969 w$ \\
\hline 91. 13 & 4 & 27084. 24 & $c{ }^{3} \mathrm{P}_{2}-551(3)$ & $(0.26) 1.13$ \\
\hline 90. 77 & 10 & 27086. 92 & a ${ }^{5} \mathrm{P}_{2}-389(3)$ & $(\mathbf{0 . 0 0 0}, 0.420,0.840) \mathbf{0 . 1 5 5}, 0.584,1.013$ \\
\hline 90. 23 & 1 & 27090.87 & $a^{3} \mathrm{D}_{2}-442(2)$ & $(0.00) 1.32 B$ \\
\hline 89. 60 & 8 & 27095. 52 & & \\
\hline 89. 25 & $\begin{array}{l}3 \\
?\end{array}$ & 27098. 04 & & $(\mathbf{0 . 0 0}, 0.59) \cdots, 1.41, \mathbf{1 . 7 4}$ \\
\hline $\begin{array}{l}\text { 88. } 25 \\
\text { 82. } 22\end{array}$ & $\begin{array}{r}2 \\
12\end{array}$ & $\begin{array}{l}27105.41 \\
27149.82\end{array}$ & $c{ }^{3} \mathrm{P}_{0}-505(1)$ & $(0.000) 1.359$ \\
\hline 79. 83 & 3 & 27167.47 & $b^{3} \mathrm{D}_{3}-577(2)$ & $(\mathbf{0 . 0 0 0 , 0 . 2 0 2 , 0 . 4 3 6 )} \cdot \ldots, 1.464, \mathbf{1 . 6 6 6}$ \\
\hline 76. 09 & $10 c$ & 27195.05 & $\left(\begin{array}{ll}b & 1 \\
{ }^{1} G_{4}-525(3) \\
a^{3} G_{3}-389(3)\end{array}\right)$ & $(0.289 W) 0.941 w$ \\
\hline $\begin{array}{l}\text { 73. } 28 \\
73.07 \\
70.96\end{array}$ & $\begin{array}{l}8 \\
4 \\
5\end{array}$ & $\begin{array}{l}27215.91 \\
27217.42 \\
27233.07\end{array}$ & $\begin{array}{lll}b^{3} \mathrm{G}_{4} & 553(4) \\
d^{3} \mathrm{~F}_{2}-570(3) \\
b^{3} \mathrm{D}_{1}-571(1)\end{array}$ & $(0.109) 1.028 w$ \\
\hline 69. 84 & 2 & 27241.42 & & $(0.00) 0.90$ \\
\hline 67.08 & 10 & 27261.89 & $a^{3} \mathrm{D}_{2}-444(3)$ & \\
\hline 62.34 & 50 & 27297. 19 & $b^{3} \mathrm{~F}_{2}-369(1)$ & $(\mathbf{0 . 0 0 0 ,} 0.377) 0.393,0.687,1.055, \mathbf{1 . 4 2 4}$ \\
\hline $\begin{array}{l}\text { 59. } 18 \\
58.40\end{array}$ & $\begin{array}{l}6 \\
2\end{array}$ & $\begin{array}{l}27320.72 \\
27326.56\end{array}$ & $b^{3} \mathrm{H}_{4}-517(5)$ & $(0.00 W) 1.52 B$ \\
\hline 56. 64 & 5 & 27339. 72 & $b^{3} \mathrm{G}_{4}-555(5)$ & $(\mathbf{0 . 0 0 0}, 0.459) 1.119$ \\
\hline 56.51 & 10 & 27340.71 & & \\
\hline 54.81 & 50 & 27353. 41 & $\begin{array}{l}a^{5} \mathrm{D}_{4}-445(5) \\
b^{3} \mathrm{G}-542(4)\end{array}$ & $(0.000 W) 1.440 W$ \\
\hline 51.54 & 7 & 27377.87 & $\begin{array}{l}0 \\
b^{3} \mathrm{G}_{3}-542(4) \\
{ }^{3} \mathrm{G}_{4}-555(3)\end{array}$ & \\
\hline 49. 32 & $7 c$ & 27394.58 & $a^{5} \mathrm{D}_{4}-446(4)$ & $(0.145, \mathbf{0 . 5 2 2}) 1.201 w$ \\
\hline 48. 18 & 4 & 27403.14 & $d^{3} \mathrm{P}_{1}-593(2)$ & $(0.00) 1.29$ \\
\hline 45. 88 & 10 & 27420.41 & $a^{5} \mathrm{P}_{2}-392(3)$ & $(\mathbf{0 . 0 0 0}, 0.284) \mathbf{0 . 5 7 4}, 0.866,1.140$ \\
\hline 45. 44 & 20 & 27423. 69 & & $(0.000 \mathrm{~W}) 1.330$ \\
\hline 44. 31 & 25 & 27432. 20 & $b^{3} \mathrm{H}_{5}-528(6)$ & $(\mathbf{0 . 0 0 0}, 0.098,0.206,0.309,0.418,0.517) 1.393,1.464,1.585, \mathbf{1 . 6 7 5}$ \\
\hline 41. 47 & 35 & 27453. 60 & $c{ }^{3} \mathrm{~F}_{3}-510(3)$ & $(0.260 B) 1.106 \mathrm{w}$ \\
\hline 40. 79 & 3 & 27458. 71 & & $(0.000 \mathrm{~W}) 1.243 \mathrm{w}$ \\
\hline 37. 69 & $7 c$ & 27482. 11 & & $(0.000 w) 0.786$ \\
\hline 34. 34 & 2 & 27507. 46 & $c{ }^{3} \mathrm{P}_{2}-555(2)$ & \\
\hline 31. 93 & 25 & 27525.72 & $a^{3} \mathrm{D}_{1}-421(2)$ & $(\mathbf{0 . 0 0 0}, 0.369) \quad 0.838,1.207, \mathbf{1 . 5 7 3}$ \\
\hline 31.71 & 20 & 27527. 41 & $a{ }^{1} \mathrm{H}_{5}-517(5)$ & $(0.355,0.473, \mathbf{0 . 5 9 3}) \cdot \cdots \mathbf{0 . 9 8 8 , 1 . 1 0 2 , 1 . 2 0 1}$ \\
\hline 31. 48 & 10 & 27529.14 & $a^{3} \mathrm{G}_{3}-392(3)$ & $(0.517, \mathbf{0 . 7 6 2}) 0.687, \mathbf{0 . 9 1 4}, \mathbf{1 . 1 3 9}, 1.380$ \\
\hline 30. 01 & 15 & 27540.25 & $b^{3} \mathrm{~F}_{2}-372(2)$ & $(0.438, \mathbf{0 . 8 6 7}) 0.185, \mathbf{0 . 6 1 3}, \mathbf{1 . 0 5 0}, 1.462$ \\
\hline 28. 68 & $50 c$ & 27550.41 & & $(0.407 B) 0.782,1.583 W$ \\
\hline 26. 10 & $\stackrel{5}{5}$ & 27569.95 & $a^{1} \mathrm{G}_{4}-417(4)$ & \\
\hline 24. 17 & 25 & 27584. 64 & $a^{1} \mathrm{D}_{2}-411(2)$ & $(0.101 B) 1.129 w$ \\
\hline
\end{tabular}


TABLE 1. Wavelengths, term combinations, and Zeeman effects of Ta II-Continued

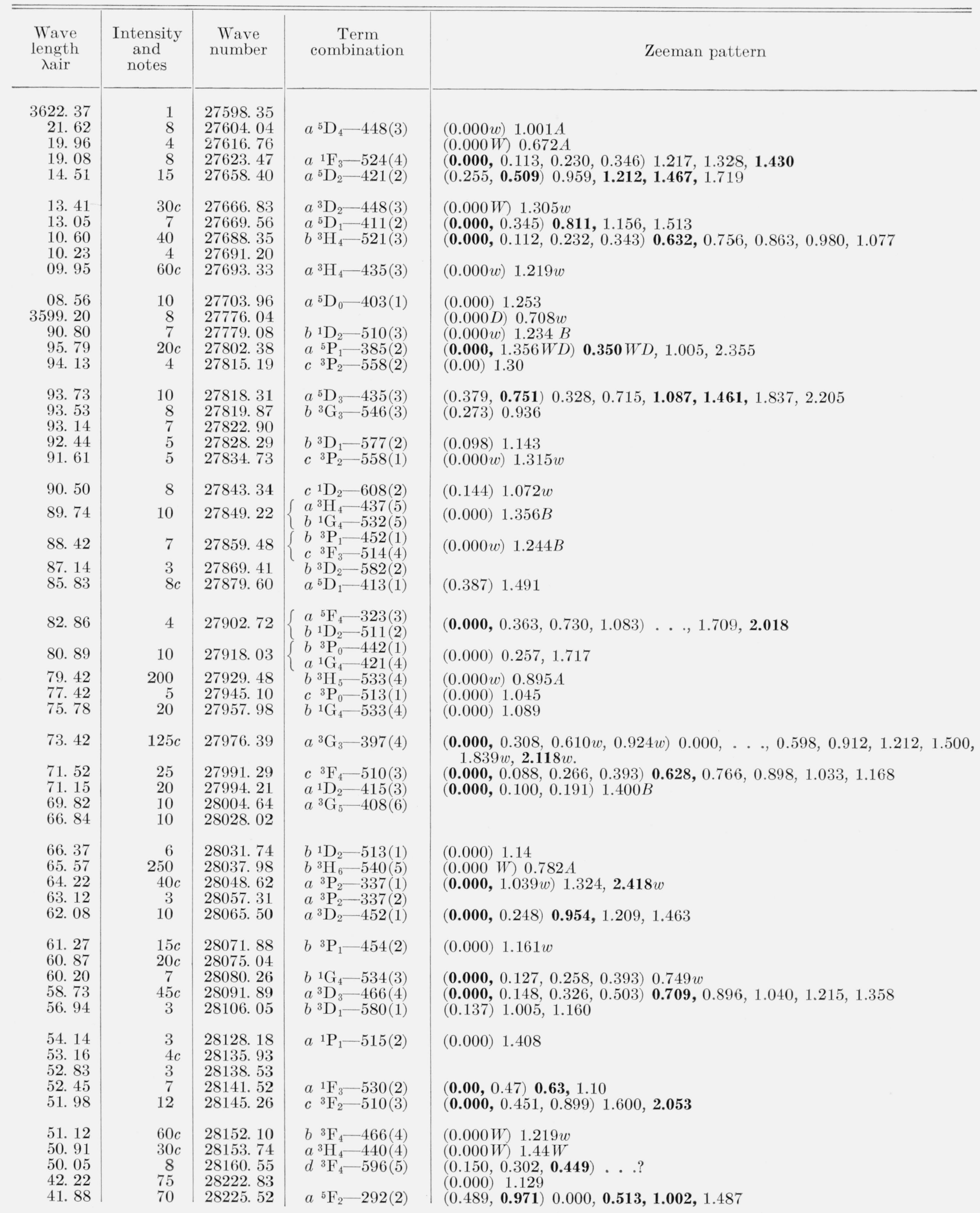


TABLE 1. Wavelengths, term combinations, and Zeeman effects of Ta II-Continued

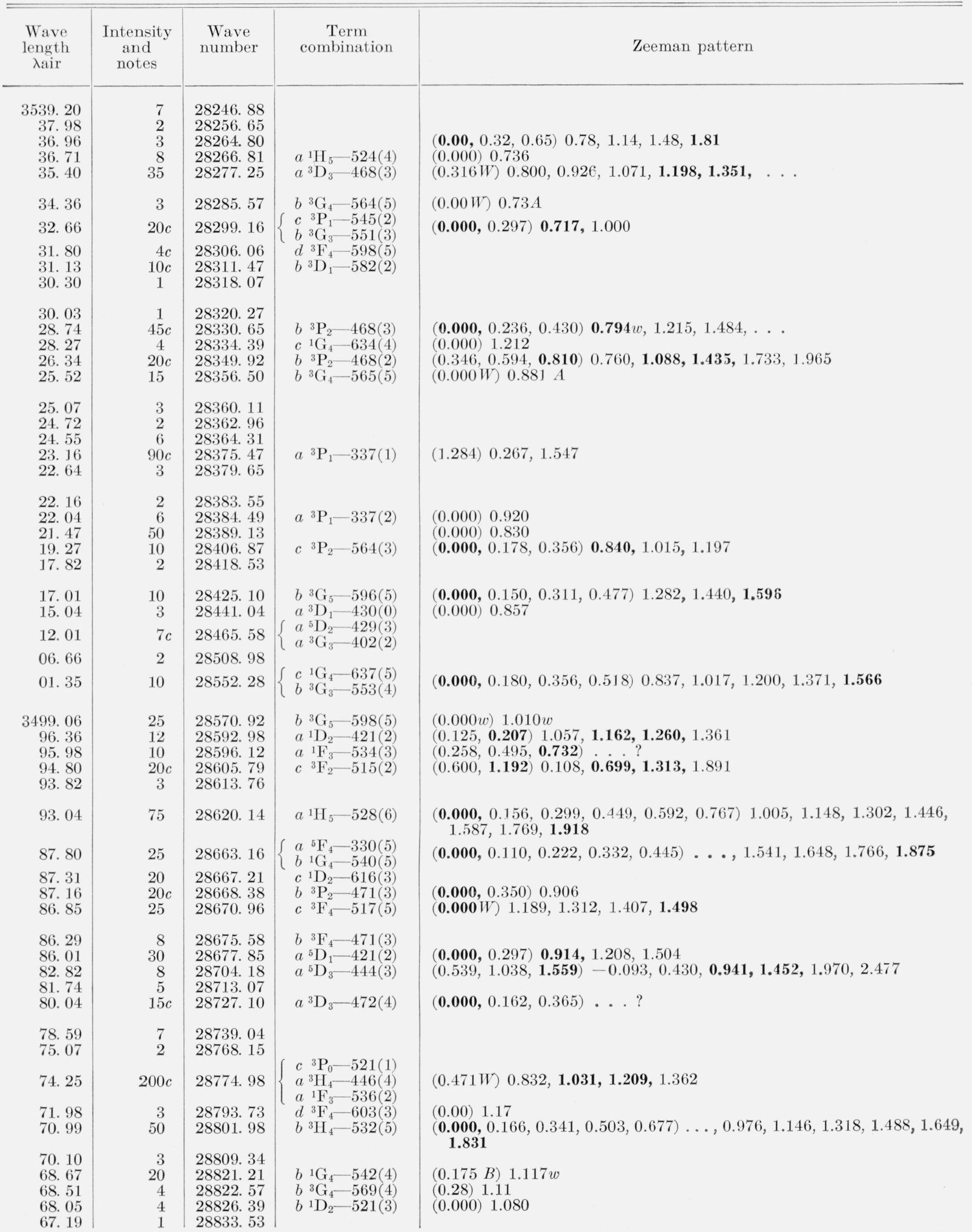


TABLE 1. Wavelengths, term combinations, and Zeeman effects of Ta II-Continued

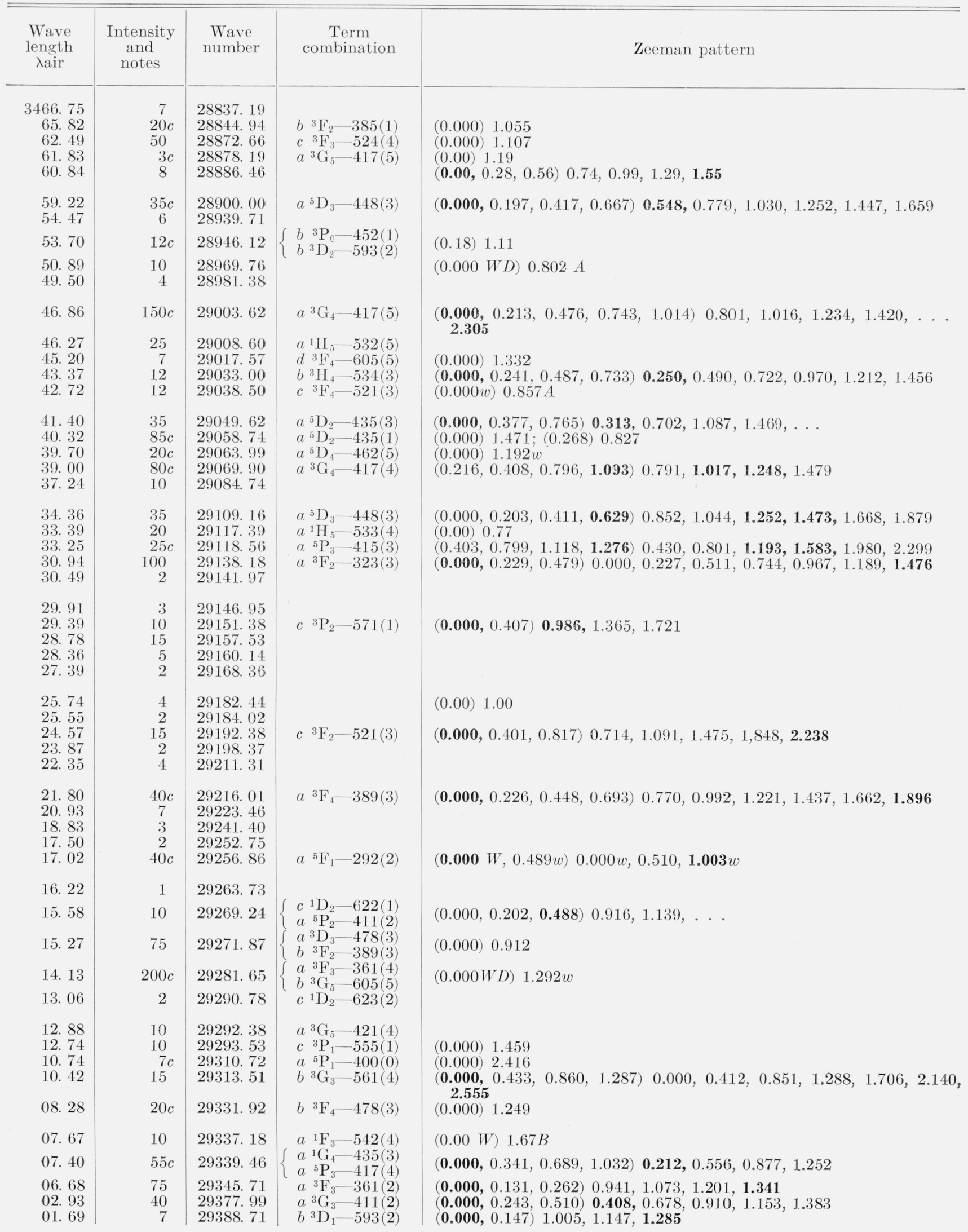


TABLE 1. Wavelengths, term combinations, and Zeeman effects of Ta II-Continued

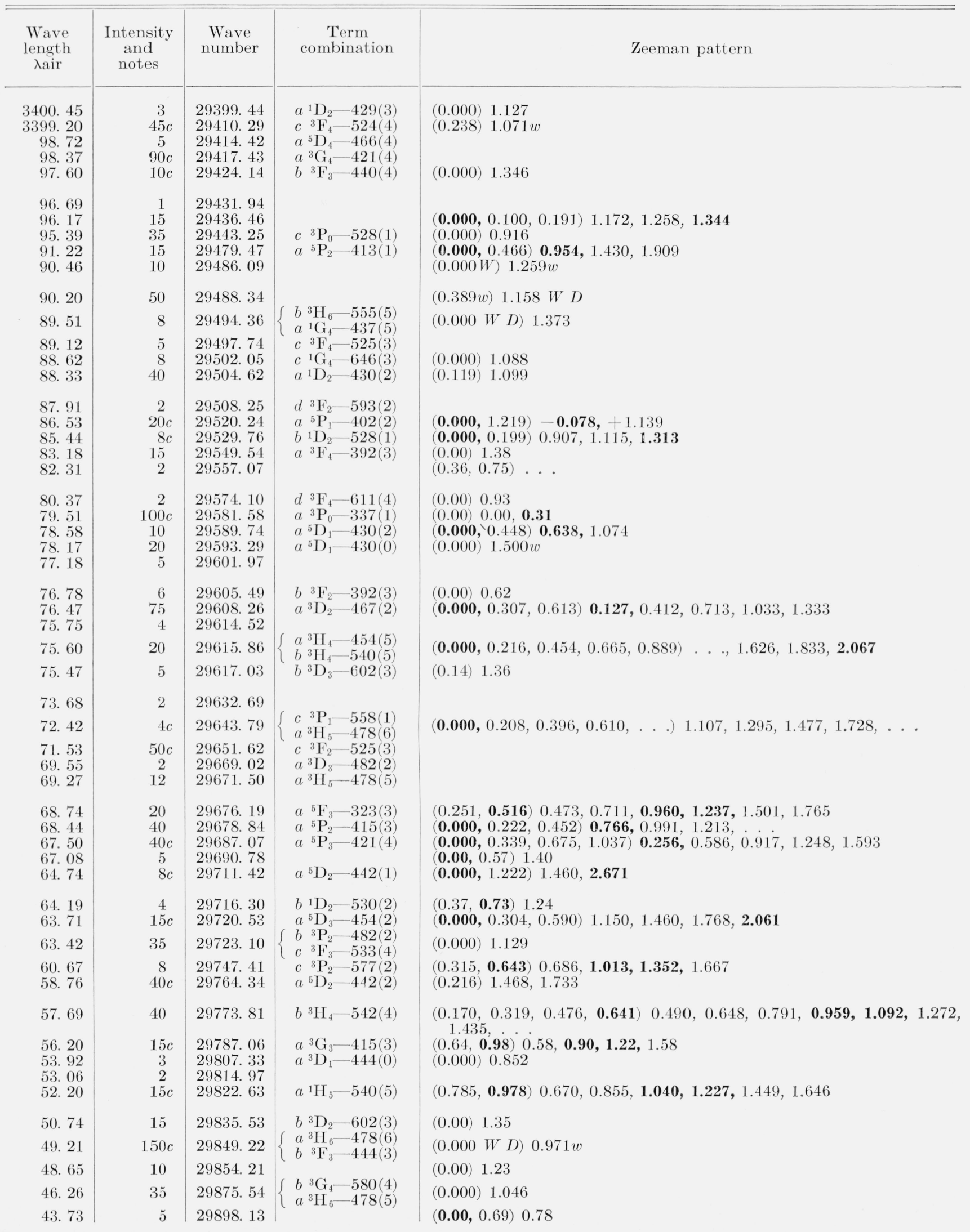


TABLE 1. Wavelengths, term combinations, and Zeeman effects of $\mathrm{Ta} \mathrm{II}$-Continued

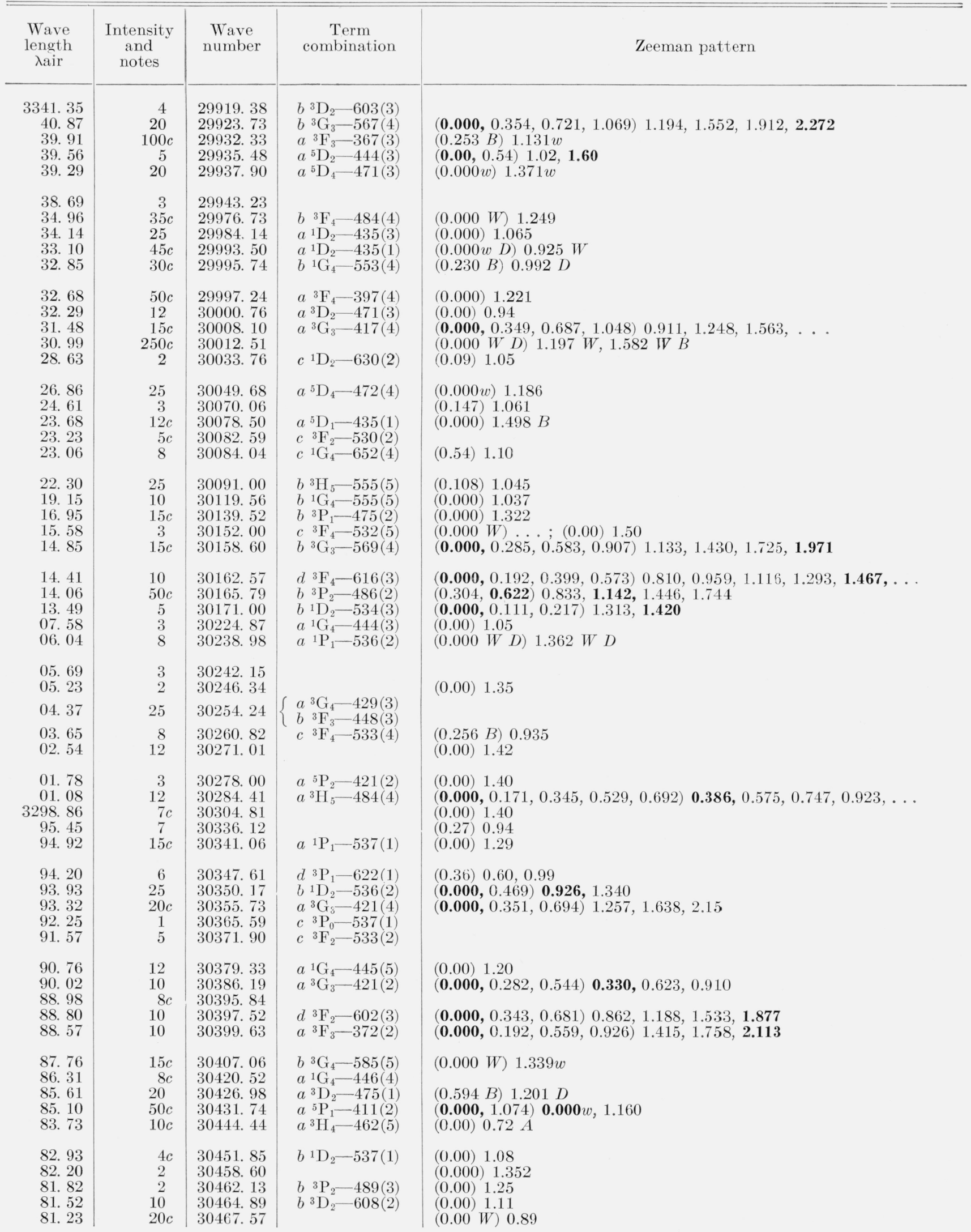


TABLE 1. Wavelengths, term combinations, and Zeeman effects of Ta II-Continued

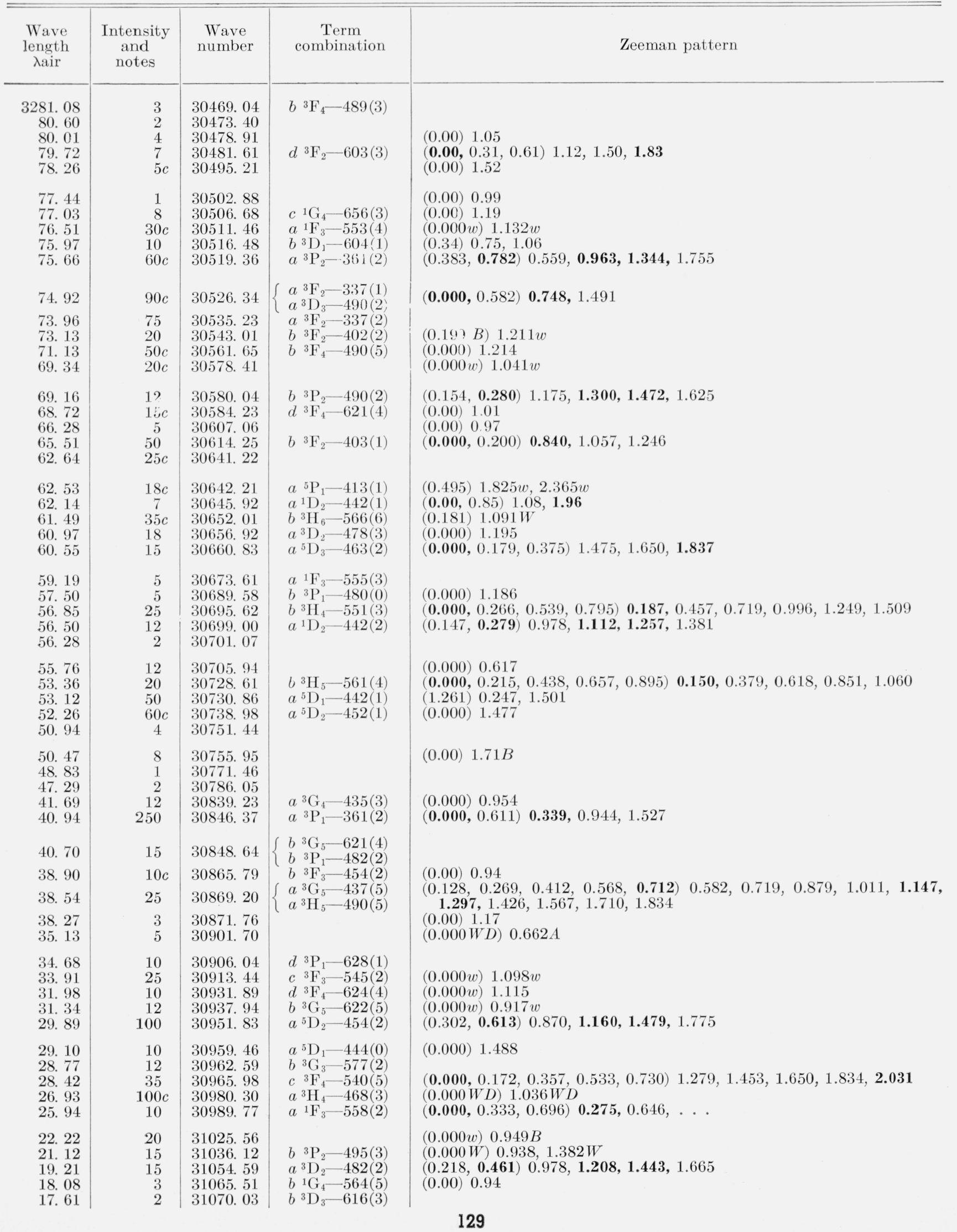


TABLE 1. Wavelengths, term combinations, and Zeeman effects of Ta II-Continued

\begin{tabular}{|c|c|c|c|c|}
\hline $\begin{array}{l}\text { Wave } \\
\text { length } \\
\text { 入air }\end{array}$ & $\begin{array}{l}\text { Intensity } \\
\text { and } \\
\text { notes }\end{array}$ & $\begin{array}{l}\text { Wave } \\
\text { number }\end{array}$ & $\begin{array}{l}\text { Term } \\
\text { combination }\end{array}$ & Zeeman pattern \\
\hline 3217.37 & 12 & 31072.27 & $\left\{\begin{array}{l}b^{3} \mathrm{H}_{4}-555(5) \\
a^{5} \mathrm{~F}_{3}-337(2)\end{array}\right.$ & $(0.000 W D) 1.228 W D$ \\
\hline $\begin{array}{l}\text { 17. } 22 \\
\text { 16. } 15 \\
\text { 15. } 32 \\
\text { 15. } 13\end{array}$ & $\begin{array}{l}6 \\
75 \\
15 c \\
10\end{array}$ & $\begin{array}{l}\text { 31073. } 71 \\
\text { 31084. } 06 \\
\text { 31092. } 16 \\
\text { 31093. } 99\end{array}$ & $\begin{array}{l}a \\
{ }^{5} \mathrm{P}_{2}-429(3) \\
b \\
{ }^{3} \mathrm{P}_{2}-495(2)\end{array}$ & $\begin{array}{l}(0.00) 1.20 \\
(\mathbf{0 . 0 0 0 , 0 . 3 0 0 , 0 . 6 1 5 )} \mathbf{0 . 5 1 2}, 0.818,1.114,1.396 \\
(0.00 W) 1.54 \\
(0.00) 1.33\end{array}$ \\
\hline 13. 91 & 150 & 31105.80 & $\left\{\begin{array}{l}a^{5} \mathrm{D}_{3}-468(3) \\
a^{3} \mathrm{P}_{2}-367(3)\end{array}\right.$ & $(0.000,0.172,0.364) \quad 0.788,1.012, \ldots$ \\
\hline $\begin{array}{l}\text { 13. } 70 \\
\text { 13. } 29\end{array}$ & $\begin{array}{r}20 \\
1\end{array}$ & $\begin{array}{l}31107.78 \\
31111.80\end{array}$ & $\begin{array}{l}b^{3} \mathrm{H}_{5}-565(5) \\
d^{3} \mathrm{P}_{1}-630(2)\end{array}$ & $(0.31) 1.13 D$ \\
\hline 12. 00 & 2 & 31124. 22 & $\left\{\begin{array}{l}c{ }^{3} \mathrm{~F}_{4}-542(4) \\
a^{5} \mathrm{D}_{3}-468(2)\end{array}\right.$ & \\
\hline 11. 62 & 2 & 31127.89 & $a^{1} \mathrm{P}_{1}-545(2)$ & $(0.000) 1.027$ \\
\hline $\begin{array}{l}\text { 10. } 73 \\
09.76 \\
09.42 \\
09.03 \\
08.83\end{array}$ & $\begin{array}{l}10 \\
50 c \\
5 \\
25 \\
10\end{array}$ & $\begin{array}{l}31136.58 \\
31145.98 \\
31149.30 \\
31153.08 \\
31155.00\end{array}$ & $\begin{array}{ll}b & 1 \mathrm{G}_{4}-565(5) \\
b & { }^{3} \mathrm{P}_{2}-496(3) \\
a & 1{ }^{1} \mathrm{~F}_{3}-560(2) \\
b & { }^{3} \mathrm{~F}_{4}-496(3) \\
a^{1} \mathrm{H}_{5}-553(4)\end{array}$ & 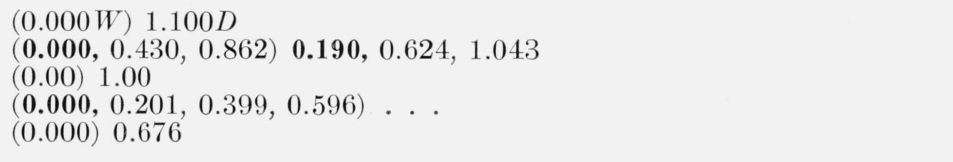 \\
\hline $\begin{array}{l}08.08 \\
05.28 \\
04.58 \\
02.96\end{array}$ & $\begin{array}{c}1 \\
20 c \\
10 \\
2\end{array}$ & $\begin{array}{l}31162.31 \\
31189.49 \\
31196.28 \\
31212.08\end{array}$ & $\begin{array}{l}a{ }^{5} \mathrm{P}_{2}-430(2) \\
b^{3} \mathrm{G}_{5}-624(4) \\
a^{5} \mathrm{~F}_{1}-312(1)\end{array}$ & $(0.000) 1.087$ \\
\hline 00.18 & $90 c$ & 31239.23 & $\left\{\begin{array}{l}b^{1} \mathrm{D}_{2}-545(2) \\
a^{5} \mathrm{D}_{4}-484(4)\end{array}\right.$ & $(0.183 W, 0.228) 1.200 W D$ \\
\hline 3199. 21 & 60 & 31248. 70 & $b^{3} \mathrm{H}_{5}-566(6)$ & $(0.000 w 1.271 B$ \\
\hline 98. 93 & 75 & 31251. 43 & & $(0.000 w) 0.885 A$ \\
\hline & 8 & 31273.06 & $a^{1} \mathrm{~F}_{3}-561(4)$ & \\
\hline $\begin{array}{l}96.52 \\
96.08\end{array}$ & $\begin{array}{l}15 \\
25 c\end{array}$ & $\begin{array}{l}31275.02 \\
31279.32\end{array}$ & $\begin{array}{l}a^{1} \mathrm{D}_{2}-448(3) \\
a^{1} \mathrm{H}_{5}-555(5)\end{array}$ & 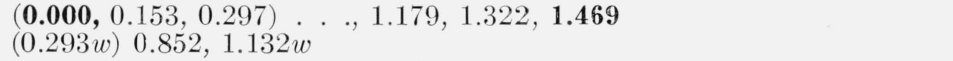 \\
\hline $\begin{array}{l}\text { 95. } 28 \\
\text { 95. } 13 \\
94.83 \\
94.19 \\
93.97\end{array}$ & $\begin{array}{l}20 c \\
6 \\
75 c \\
2 \\
7 c\end{array}$ & $\begin{array}{l}31287.12 \\
31288.63 \\
31291.48 \\
31297.83 \\
31299.97\end{array}$ & $\begin{array}{l}a{ }^{5} \mathrm{~F}_{2}-323(3) \\
b^{3} \mathrm{D}_{2}-616(3) \\
b{ }^{3} \mathrm{P}_{1}-486(2) \\
a^{3} \mathrm{G}_{3}-430(2) \\
a^{3} \mathrm{G}_{4}-440(4)\end{array}$ & $\begin{array}{l}(0.000 w) 0.940 w \\
(0.00) 0.86 \\
(0.000) 1.114 \\
(0.129,0.280,0.437, \mathbf{0 . 5 6 9}) 1.032 W D\end{array}$ \\
\hline 93. 16 & 15 & 31307.89 & $\begin{cases}c & { }^{3} \mathrm{P}_{2}-593(2) \\
b & 3 \mathrm{P}_{0}-475(1)\end{cases}$ & $(0.000) 1.502$ \\
\hline $\begin{array}{l}92.55 \\
90.92 \\
90.00 \\
89.19\end{array}$ & $\begin{array}{r}2 \\
7 \\
7 \\
20\end{array}$ & $\begin{array}{l}31313.89 \\
31329.85 \\
31338.94 \\
31346.87\end{array}$ & $\begin{array}{l}a{ }^{3} \mathrm{P}_{2}-369(1) \\
b^{3} \mathrm{H}_{5}-567(4)\end{array}$ & 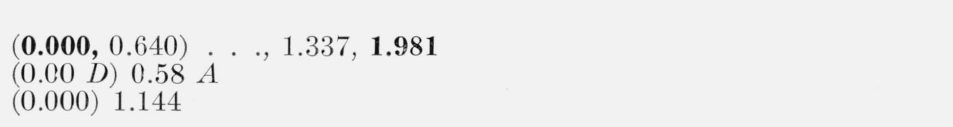 \\
\hline $\begin{array}{l}\text { 87. } 09 \\
85.17 \\
84.73 \\
82.95 \\
79.73\end{array}$ & $\begin{array}{l}10 \\
25 c \\
2 \\
8 \\
25 c\end{array}$ & $\begin{array}{l}\text { 31367. } 48 \\
31386.39 \\
31390.74 \\
31408.33 \\
31440.12\end{array}$ & $\begin{array}{ll}b & { }^{1} \mathrm{G}_{4}-567(4) \\
b & { }^{3} \mathrm{P}_{2}-498(1)\end{array}$ & $\begin{array}{l}(0.380 w) 1.146 w D \\
(\mathbf{0 . 0 0 0}, 0.548) \mathbf{0 . 9 0 5} w, 1.442,1.989 \\
(0.000 w) 1.109 w \\
(0.00) 1.24\end{array}$ \\
\hline $\begin{array}{l}79.44 \\
79.12 \\
77.56 \\
77.27 \\
75.53\end{array}$ & $\begin{array}{r}12 \\
8 \\
10 \\
2 \\
10\end{array}$ & $\begin{array}{l}31443.02 \\
31446.13 \\
31461.55 \\
31464.45 \\
31481.68\end{array}$ & $\begin{array}{l}a^{5} \mathrm{D}_{3}-471(3) \\
b^{3} \mathrm{G}_{3}-582(2)\end{array}$ & 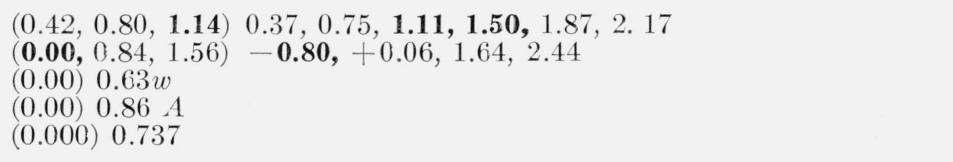 \\
\hline $\begin{array}{l}\text { 74. } 49 \\
72.88 \\
\text { 70. } 77 \\
68.96 \\
68.17\end{array}$ & $\begin{array}{l}2 \\
25 \\
8 \\
15 c \\
75\end{array}$ & $\begin{array}{l}\text { 31492. } 05 \\
31508.01 \\
31529.00 \\
31547.01 \\
31554.81\end{array}$ & $\begin{array}{l}b^{3} \mathrm{D}_{3}-621(4) \\
c^{3} \mathrm{~F}_{3}-551(3) \\
a^{3} \mathrm{D}_{1}-461(1) \\
a^{5} \mathrm{D}_{3}-472(4)\end{array}$ & $\begin{array}{l}(0.00 W) 0.46 \\
(0.00) 1.14 \\
(0.517) 0.912,1.390 \\
(\mathbf{0 . 0 0 0}, 0.271,0.503,0.836) \mathbf{0 . 3 6 7}, 0.653,0.919,1.197,1.468,1.727,1.988\end{array}$ \\
\hline $\begin{array}{l}\text { 66. } 71 \\
\text { 66. } 36 \\
65.80 \\
65.50 \\
64.34\end{array}$ & $\begin{array}{l}50 c \\
40 \\
4 c \\
3 \\
15\end{array}$ & $\begin{array}{l}\text { 31569. } 40 \\
31572.83 \\
31578.43 \\
31581.47 \\
31592.97\end{array}$ & $\begin{array}{ll}a & { }^{5} \mathrm{P}_{3}-440(4) \\
a & { }^{3} \mathrm{P}_{2}-372(2)\end{array}$ & $\begin{array}{l}(\mathbf{0 . 0 0 0}, 0.491,0.972,1.457)-0.364,+0.141,0.639, \mathbf{1 . 1 1 8} \\
(0.000,0.719, \mathbf{1 . 4 3 6})-0.113, \mathbf{0 . 6 1 0}, \mathbf{1 . 3 3 3 , 2 . 0 5 3} \\
(0.433, \mathbf{0 . 6 5 3}) \ldots . ? \\
(0.00) 1.19\end{array}$ \\
\hline
\end{tabular}


TABLE 1. Wavelengths, term combinations, and Zeeman effects of Ta II-Continued

\begin{tabular}{|c|c|c|c|c|}
\hline $\begin{array}{l}\text { Wave } \\
\text { length } \\
\quad \lambda \text { air }\end{array}$ & $\begin{array}{l}\text { Intensity } \\
\text { and } \\
\text { notes }\end{array}$ & $\begin{array}{l}\text { Wave } \\
\text { number }\end{array}$ & $\begin{array}{l}\text { Term } \\
\text { combination }\end{array}$ & Zeeman pattern \\
\hline 3163. 12 & $40 c$ & 31605.22 & \multirow{2}{*}{$\begin{array}{l}c{ }^{3} \mathrm{~F}_{2}-545(2) \\
a^{5} \mathrm{D}_{0}-442(1) \\
a^{3} \mathrm{D}_{2}-487(1)\end{array}$} & $(\mathbf{0 . 0 9 8}, 0.316) \mathbf{1 . 0 7 1}, 1.269,1.454$ \\
\hline $\begin{array}{l}62.86 \\
61.90\end{array}$ & $\begin{array}{c}20 c \\
2\end{array}$ & $\begin{array}{l}31607.86 \\
31617.36\end{array}$ & & $(\mathbf{0 . 0 0 0 ,} 0.428) 0.712,1.149, \mathbf{1 . 5 7 6}$ \\
\hline 61. 72 & 4 & 31619.20 & \multirow[b]{2}{*}{$a^{3} \mathrm{P}_{1}-369(1)$} & \\
\hline 57.95 & $70 c$ & 31656.95 & & $(0.877) 0.670,1.546$ \\
\hline 57. 64 & $50 c$ & 31660.03 & \multirow{4}{*}{$\begin{array}{ll}b & 3 \mathrm{~F}_{2}-413(1) \\
a & { }^{5} \mathrm{P}_{2}-435(3)\end{array}$} & $(0.447,0.675, \mathbf{0 . 9 2 9}) 0.582,0.818,1.039, \mathbf{1 . 2 6 7}, 1.504,1.743,1.949$ \\
\hline 57. 19 & 5 & 31664.59 & & $(\mathbf{0 . 0 0}, 0.86) 0.23, \ldots$ \\
\hline 56. 74 & $50 \mathrm{c}$ & 31669.05 & & $(\mathbf{0 . 0 0 0}, 0.356,0.698) \mathbf{0 . 4 0 8} w, 0.750,1.075$ \\
\hline $\begin{array}{l}56.58 \\
56.37\end{array}$ & $\begin{array}{r}10 \\
2\end{array}$ & $\begin{array}{l}31670.65 \\
31672.79\end{array}$ & & $\begin{array}{ll}(0.00) & 1.04) \\
(0.00) & 1.18\end{array}$ \\
\hline 56. 14 & 4 & 31675. 10 & \multirow{3}{*}{$\begin{array}{l}b^{1} \mathrm{G}_{4}-570(3) \\
a^{5} \mathrm{D}_{2}-461(1)\end{array}$} & \\
\hline 55. 69 & 6 & 31679.66 & & $(0.000 w) 1.498 A$ \\
\hline 55.35 & 5 & 31682.99 & & (0.00) 1.17 \\
\hline 55. 24 & 50 & 31684. 16 & \multirow{2}{*}{$\begin{array}{l}a{ }^{3} \mathrm{~F}_{3}-385(2) \\
a \\
{ }^{5} \mathrm{~F}_{4}-361(4)\end{array}$} & $(0.000 W D) 1.181 w B$ \\
\hline & 12 & 31697.42 & & $(. ., 0.334,0.509,0.696) \ldots, 1.484,1.662,1.839$ \\
\hline 53. 76 & $15 c$ & 31698.98 & \multirow{4}{*}{$\begin{array}{l}b{ }^{3} \mathrm{~F}_{3}-462(4) \\
b^{3} \mathrm{H}_{4}-561(4) \\
c^{3} \mathrm{~F}_{2}-546(3) \\
a^{3} \mathrm{G}_{4}-444(3)\end{array}$} & $(0.00 W) 0.92$ \\
\hline 53. 07 & $5 c$ & 31705.94 & & $(0.000 W) 1.109 A$ \\
\hline 52.69 & 7 & 31709.78 & & $(0.96,1.20) \cdot .1 .15,1.55,1.83,2.23$ \\
\hline $\begin{array}{l}\text { 51. } 65 \\
51.16\end{array}$ & $\begin{array}{l}15 \\
50 c\end{array}$ & $\begin{array}{l}31720.21 \\
31725.12\end{array}$ & & $\begin{array}{l}(0.000,0.227,0.468) 0.716,0.942,1.160 \\
(0.000 W) 1.182 w\end{array}$ \\
\hline 50.54 & $20 c$ & 31731.37 & \multirow{5}{*}{$\begin{array}{l}a^{5} \mathrm{D}_{4}-489(3) \\
a^{3} \mathrm{D}_{1}-463(2) \\
a^{3} \mathrm{G}_{3}-435(3) \\
a^{5} \mathrm{D}_{3}-475(2) \\
a^{3} \mathrm{D}_{2}-489(3)\end{array}$} & $(0.000 W D) 1.057 w$ \\
\hline 47. 70 & $20 c$ & 31760.03 & & $(0.00) 0.210 B$ \\
\hline 45. 99 & $20 c$ & 31777.28 & & $(0.166,0.337, \mathbf{0 . 5 3 1}) 0.651, \mathbf{0 . 8 3 4}, \mathbf{1 . 0 0 8}, 1.257$ \\
\hline $\begin{array}{l}\text { 44. } 86 \\
44.32\end{array}$ & $30 c$ & $\begin{array}{l}31788.70 \\
3170413\end{array}$ & & $(\mathbf{0 . 0 0 0 ,} 0.169,0.361) 1.288,1.462,1.631, \mathbf{1 . 8 2 4}$ \\
\hline & 10 & 31794.13 & & $(0.000) 1.358 B$ \\
\hline 42. 95 & 75 & 31808. 07 & \multirow{4}{*}{$\begin{array}{ll}a & { }^{3} \mathrm{~F}_{4}-415(3) \\
b & { }^{3} \mathrm{P}_{2}-503(3) \\
b & { }^{3} \mathrm{~F}_{4}-503(3) \\
a^{5} \mathrm{D}_{4}-490(5) \\
c \\
c^{3} \mathrm{P}_{1}-580(1)\end{array}$} & $(0.000) 1.243$ \\
\hline 42. 36 & $20 c$ & 31814. 01 & & $(\mathbf{0 . 0 0 0}, 0.201,0.410) \mathbf{0 . 7 7 2}, 1.039$ \\
\hline 41. 69 & $\begin{array}{c}8 \\
80 c\end{array}$ & $\begin{array}{l}31820.77 \\
3182400\end{array}$ & & $\begin{array}{l}(0.000) 1.226 \\
(0.000 w)\end{array}$ \\
\hline 40. 33 & $\begin{array}{c}80 c \\
4\end{array}$ & 31834.56 & & \\
\hline 39.84 & 20 & 31839.57 & \multirow{3}{*}{$\begin{array}{l}b^{3} \mathrm{D}_{3}-624(4) \\
d^{3} \mathrm{~F}_{2}-616(3)\end{array}$} & $(0.268 w) 1.193 w$ \\
\hline 38. 75 & 7 & 31850.63 & & $(0.000 W D) 1.042$ \\
\hline 38.04 & 5 & 31857.76 & & $(0.000) 1.026$ \\
\hline 37. 43 & 75 & 31863.95 & \multirow{2}{*}{$\begin{array}{l}a^{3} \mathrm{D}_{1}-464(2) \\
b{ }^{3} \mathrm{~F}_{2}-415(3)\end{array}$} & $(\mathbf{0 . 0 0 0}, 0.163,0.331) 0.892,1.051,1.198,1.358, \mathbf{1 . 5 2 1}$ \\
\hline 36. 72 & 4 & 31871. 19 & & $(0.33) 1.67$ \\
\hline 35.83 & $120 c$ & $\begin{array}{l}31880.22 \\
31883.30\end{array}$ & \multirow{4}{*}{$\begin{array}{l}a^{3} \mathrm{G}_{4}-445(5) \\
a^{1} \mathrm{~F}_{3}-567(4) \\
a^{1} \mathrm{D}_{2}-454(2) \\
b^{3} \mathrm{D}_{2}-622(1) \\
a^{3} \mathrm{P}_{1}-372(2)\end{array}$} & $(\mathbf{0 . 0 0 0}, 0.253,0.551,0.877,1.187) 1.017,1.272,1.525,1.860,2.153,2.469$ \\
\hline $\begin{array}{l}35.53 \\
35.20\end{array}$ & $\begin{aligned} 5 \\
15\end{aligned}$ & 31886.65 & & $(0.123) 1.111 w$ \\
\hline 34. 80 & 5 & 31890.71 & & $(\mathbf{0 . 0 0}, 0.48) 0.58,1.13, \mathbf{1 . 5 5}$ \\
\hline 33. 89 & 50 & 31899.95 & & 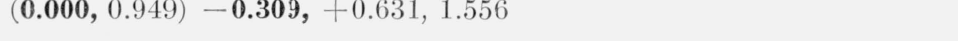 \\
\hline 32.86 & 3 & 31910.46 & \multirow{4}{*}{$\begin{array}{l}b{ }^{3} \mathrm{~F}_{3}-464(2) \\
a^{3} \mathrm{G}_{4}-446(4) \\
c c^{3} \mathrm{~F}_{3}-555(3) \\
c{ }^{3} \mathrm{~F}_{3}-555(2) \\
d^{3}{ }^{3} \mathrm{~F}_{4}-634(4)\end{array}$} & \\
\hline 31.85 & $90 c$ & 31920.73 & & $(0.220,0.772) \cdots ?$ \\
\hline $\begin{array}{l}31.60 \\
30.80\end{array}$ & $\begin{array}{l}90 \mathrm{c} \\
8\end{array}$ & $\begin{array}{l}31920.33 \\
31931.46\end{array}$ & & $(0.00 w) 1.13$ \\
\hline 28.59 & 10 & 31954.04 & & $(0.29) 1.10 \mathrm{w}$ \\
\hline 27. 74 & $100 c$ & 31962. 74 & \multirow{5}{*}{$\begin{array}{l}a^{3} \mathrm{H}_{4}-417(5) \\
a^{5} \mathrm{D}_{1}-454(2) \\
a^{3} \mathrm{H}_{4}-478(3) \\
a^{5} \mathrm{P}_{3}-444(3) \\
a^{3} \mathrm{H}_{4}-478(5)\end{array}$} & $(0.000 w) 1.264 w$ \\
\hline 26. 87 & 7 & 31971.58 & & $(\mathbf{0 . 0 0 0 ,} 0.341) \mathbf{0 . 7 8 1}, 1.148,1.503$ \\
\hline 26. 59 & $7 c$ & 31974.45 & & $(0.000 w) 1.107$ \\
\hline 24. 62 & $50 c$ & 31994.66 & & $(0.670,1.326, \mathbf{1 . 9 7 6} w) \ldots 0.327, \mathbf{0 . 9 3 6}, \mathbf{1 . 5 6 0}, 2.237,2.912$ \\
\hline 23. 46 & 3 & 32006.55 & & \\
\hline 22. 78 & $15 c$ & 32013.42 & \multirow{5}{*}{$\begin{array}{ll}b & { }^{3} \mathrm{~F}_{4}-505(5) \\
b & { }^{3} \mathrm{H}_{4}-564(5) \\
a^{3} \mathrm{~F}_{4}-417(4) \\
b & { }^{3} \mathrm{P}_{2}-505(1) \\
c & { }^{3} \mathrm{P}_{1}-582(2)\end{array}$} & \multirow{5}{*}{$\begin{array}{l}(\mathbf{0 . 0 0 0}, 0.156,0.330) 1.17 B \\
(\mathbf{0 . 0 0 0}, 0.205,0.410,0.622) \mathbf{0 . 3 5 2}, 0.568,0.778,0.976,1.186 \\
(0.139) 1.236 A\end{array}$} \\
\hline 22. 32 & 18 & 32018. 22 & & \\
\hline 21. 26 & $40 c$ & 32029. 03 & & \\
\hline 21. 09 & $\begin{array}{l}7 \\
2\end{array}$ & $\begin{array}{l}32030.79 \\
32040\end{array}$ & & \\
\hline 20.13 & 2 & 32040.62 & & \\
\hline 19. 64 & 5 & 32045.73 & \multirow{5}{*}{$\begin{array}{r}c{ }^{3} \mathrm{~F}_{4}-551(3) \\
b^{3} \mathrm{~F}_{3}-466(4) \\
b^{3} \mathrm{G}_{4}-602(3) \\
b^{3} \mathrm{H}_{4}-565(5) \\
a^{1} \mathrm{G}_{4}-462(5)\end{array}$} & $(0.000) 1.302 B$ \\
\hline 19. 12 & 10 & 32050.99 & & $(\mathbf{0 . 0 0 0}, 0.188) 1.231,1.397, \mathbf{1 . 5 8 2}$ \\
\hline 17. 74 & 10 & 32065. 24 & & $(\mathbf{0 . 0 0 0}, 0.210,0.421,0.645) \mathbf{0 . 9 6 0}, 1.188,1.377$ \\
\hline 16. 70 & 15 & 32075.96 & & 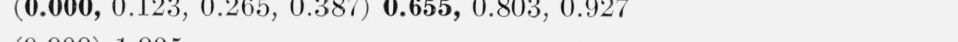 \\
\hline 15. 42 & 20 & 32089.12 & & $(0.000) 1.005$ \\
\hline
\end{tabular}


TABLE 1. Wavelengths, term combinations, and Zeeman effects of Ta II-Continued

\begin{tabular}{|c|c|c|c|c|}
\hline $\begin{array}{l}\text { Wave } \\
\text { length } \\
\text { 入air }\end{array}$ & $\begin{array}{l}\text { Intensity } \\
\text { and } \\
\text { notes }\end{array}$ & $\begin{array}{l}\text { Wave } \\
\text { number }\end{array}$ & $\begin{array}{c}\text { Term } \\
\text { combination }\end{array}$ & Zeeman pattern \\
\hline $\begin{array}{r}\text { 3114. } 45 \\
12.88 \\
12.58 \\
11.35 \\
10.81\end{array}$ & $\begin{array}{r}8 \\
50 \\
6 \\
2 \\
90 c\end{array}$ & $\begin{array}{l}\text { 32099. } 13 \\
\text { 32115. } 27 \\
\text { 32118. } 35 \\
\text { 32131. } 11 \\
32136.69\end{array}$ & $\begin{array}{l}a^{5} \mathrm{D}_{3}-478(3) \\
a^{1} \mathrm{~F}_{3}-569(4) \\
a^{3} \mathrm{~F}_{3}-389(3)\end{array}$ & $\begin{array}{l}(0.269,0.523, \mathbf{0 . 8 0 4}) 0.932, \mathbf{1 . 1 8 6}, \mathbf{1 . 4 4 7}, 1.699 \\
(0.247) 1.088 W D \\
(\mathbf{0 . 0 0 0 ,} 0.165,0.319,0.475) \ldots, 1.266,1.434, \mathbf{1 . 6 2 6} \\
(0.242) 0.633, \mathbf{0 . 8 6 5}, \mathbf{1 . 1 1 1}, 1.340 . \\
(0.263 w) 1.185 w\end{array}$ \\
\hline $\begin{array}{l}\text { 09. } 93 \\
\text { 09. } 81 \\
\text { 09. } 04 \\
\text { 08. } 58 \\
06.11\end{array}$ & $\begin{array}{l}6 c \\
10 \\
3 c \\
45 c \\
3\end{array}$ & $\begin{array}{l}32145.72 \\
32146.98 \\
32154.98 \\
32159.66 \\
32185.27\end{array}$ & $\begin{array}{l}a{ }^{1} \mathrm{P}_{1}-555(2) \\
c{ }^{3} \mathrm{P}_{0}-555(1) \\
b^{3} \mathrm{G}_{4}-603(3)\end{array}$ & 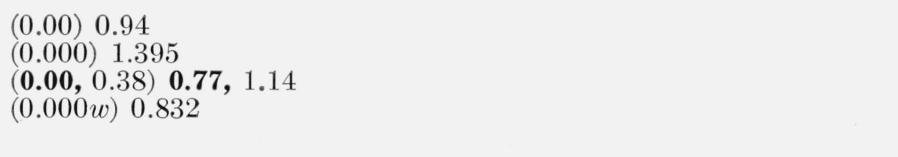 \\
\hline $\begin{array}{l}05.63 \\
04.70\end{array}$ & 50 & $\begin{array}{l}32190.20 \\
32199.89\end{array}$ & $\begin{array}{l}a{ }^{5} \mathrm{P}_{3}-446(4) \\
c^{3} \mathrm{~F}_{2}-551(3)\end{array}$ & $\begin{array}{l}(\mathbf{0 . 0 0 0}, 0.345,0.675,1.033) \mathbf{0 . 2 3 0}, 0.575,0.909,1.251,1.603 \\
(\mathbf{0 . 0 0}, 0.55,1.13) \ldots, 0.72,1.22,1.81, \mathbf{2 . 4 8}\end{array}$ \\
\hline 02.90 & 7 & 32218.56 & $\begin{cases}b & { }^{3} \mathrm{P}_{1}-495(2) \\
b & { }^{3} \mathrm{G}-634(4)\end{cases}$ & $(0.00) 1.15$ \\
\hline $\begin{array}{l}01.47 \\
01.22\end{array}$ & $\begin{array}{r}10 \\
7\end{array}$ & $\begin{array}{l}32233.46 \\
32236.02\end{array}$ & $b^{1} \mathrm{D}_{2}-555(1)$ & $(0.000,0.293) \mathbf{0 . 8 2 2}, 1.097,1.423$ \\
\hline $\begin{array}{r}01.04 \\
\text { 3099. } 83 \\
99.22 \\
97.98 \\
96.88\end{array}$ & $\begin{array}{r}100 c \\
20 c \\
2 \\
25 c \\
8 c\end{array}$ & $\begin{array}{l}32237.86 \\
32250.43 \\
32256.86 \\
32269.69 \\
32281.19\end{array}$ & 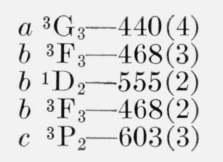 & 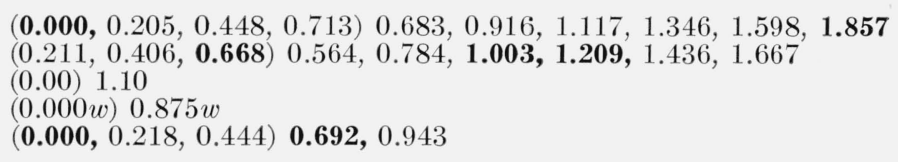 \\
\hline $\begin{array}{l}\text { 96. } 13 \\
\text { 95. } 50 \\
\text { 95. } 21 \\
94.60 \\
93.14\end{array}$ & $\begin{array}{l}10 \\
15 \\
10 \\
40 c \\
7\end{array}$ & $\begin{array}{l}32289.04 \\
32295.56 \\
32298.61 \\
32304.94 \\
32320.20\end{array}$ & $\begin{array}{l}d^{3} \mathrm{~F}_{4}-638(4) \\
a^{1} \mathrm{H}_{5}-565(5) \\
c^{3} \mathrm{~F}_{4}-553(4) \\
a^{5} \mathrm{D}_{4}-495(3) \\
b^{3} \mathrm{H}_{4}-567(4)\end{array}$ & 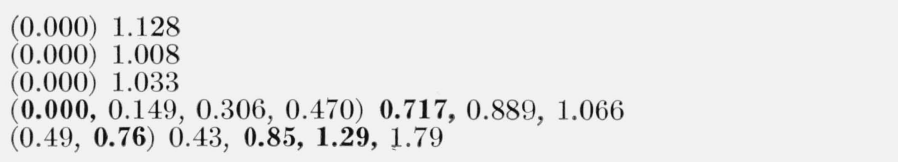 \\
\hline $\begin{array}{l}91.90 \\
91.58 \\
90.48 \\
90.14 \\
89.85\end{array}$ & $\begin{array}{l}8 \\
15 \\
10 \\
20 c \\
5\end{array}$ & $\begin{array}{l}32333.19 \\
32336.50 \\
32348.04 \\
32351.59 \\
32354.64\end{array}$ & $\begin{array}{l}b^{3} \mathrm{D}_{1}-622(1) \\
a^{5} \mathrm{D}_{2}-468(3) \\
a^{5} \mathrm{~F}_{4}-367(3) \\
a^{5} \mathrm{P}_{1}-430(2) \\
b^{3} \mathrm{D}_{1}-623(2)\end{array}$ & $\begin{array}{l}(0.39) 0.60,1.00 \\
(\mathbf{0 . 0 0 0 ,} 0.253,0.550) \mathbf{0 . 6 5 6}, 0.948,1.240 \\
(\mathbf{0 . 0 0 0 ,} 0.185,0.341,0.501) \\
(0.000) \mathbf{0 . 2 5 1}, 1.056\end{array}$ \\
\hline $\begin{array}{l}\text { 89. } 74 \\
\text { 88. } 84 \\
\text { 88. } 69 \\
\text { 88. } 60 \\
\text { 87. } 76\end{array}$ & $\begin{array}{c}15 c \\
4 \\
10 \\
10 \\
100 c\end{array}$ & $\begin{array}{l}32355.83 \\
32365.22 \\
32366.77 \\
32367.73 \\
32376.57\end{array}$ & $\begin{array}{l}a^{5} \mathrm{D}_{2}-468(2) \\
a^{3} \mathrm{D}_{2}-495(3) \\
a^{3} \mathrm{~F}_{4}-421(4)\end{array}$ & $\begin{array}{l}(0.75 B) 0.68, \mathbf{1 . 0 7}, 1.49,1.89,2.38 \\
(0.00) 1.15 \\
(0.000) 1.059 \\
(0.020 w) 1.254 w\end{array}$ \\
\hline $\begin{array}{l}\text { 87. } 08 \\
\text { 85. } 65 \\
84.06 \\
\text { 83. } 39 \\
83.19\end{array}$ & $\begin{array}{r}4 \\
10 \\
10 \\
25 \\
6\end{array}$ & $\begin{array}{l}32383.65 \\
32398.68 \\
32415.40 \\
32422.40 \\
32424.50\end{array}$ & $\begin{array}{l}a \\
{ }^{5} \mathrm{P}_{2}-442(2) \\
c \\
{ }^{3} \mathrm{~F}_{3}-560(2) \\
a^{5} \mathrm{D}_{4}-496(3) \\
c^{3} \mathrm{~F}_{4}-555(5) \\
a^{3} \mathrm{D}_{2}-495(2)\end{array}$ & $\begin{array}{l}(\mathbf{0 . 0 0 ,} 0.70) 1.13 \\
(\mathbf{0 . 0 0 0}, 0.147,0.291,0.439) 1.030,1.171,1.312,1.460, \mathbf{1 . 6 1 3} \\
(0.000 w) 0.098 w \\
(0.34, \mathbf{0 . 7 3}) 0.88, \mathbf{1 . 2 6}, \mathbf{1 . 6 4}, \ldots .\end{array}$ \\
\hline 82. 06 & $90 c$ & 32436.42 & $\begin{array}{l}b^{3} \mathrm{G}_{5}-637(5) \\
b^{3} \mathrm{D}_{5}-630(2) \\
a^{1} \mathrm{H}_{5}-566(6)\end{array}$ & $(0.00 w) 1.40 w$ \\
\hline $\begin{array}{l}80.53 \\
79.56 \\
79.40 \\
78.47\end{array}$ & $\begin{array}{c}1 \\
75 c \\
4 \\
10\end{array}$ & $\begin{array}{l}32452.55 \\
32462.80 \\
32464.43 \\
32474.19\end{array}$ & $\begin{array}{l}d^{3} \mathrm{~F}_{2}-622(1) \\
b{ }^{3} \mathrm{~F}_{2}-421(2) \\
a^{3} \mathrm{~F}_{3}-392(3) \\
d^{3} \mathrm{~F}_{2}-623(2)\end{array}$ & 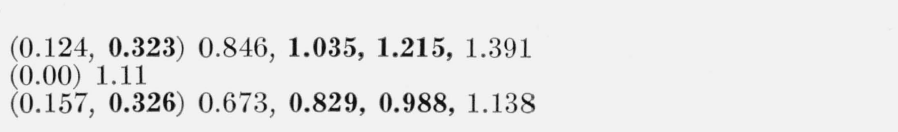 \\
\hline $\begin{array}{l}\text { 78. } 10 \\
\text { 76. } 80 \\
\text { 76. } 32 \\
\text { 75. } 31 \\
74.90\end{array}$ & $\begin{array}{c}25 c \\
8 c \\
20 \\
2 \\
20 c\end{array}$ & $\begin{array}{l}32478.17 \\
32491.88 \\
32496.89 \\
32507.60 \\
32511.94\end{array}$ & $\begin{array}{l}a^{3} \mathrm{D}_{2}-496(3) \\
a^{3} \mathrm{G}_{3}-442(2) \\
a^{5} \mathrm{D}_{3}-482(2) \\
a^{3} \mathrm{H}_{5}-506(6) \\
b{ }^{3} \mathrm{P}_{1}-498(1)\end{array}$ & $\begin{array}{l}(0.58) 0.71 w \\
(\mathbf{0 . 0 0}, 0.39) \ldots ? \\
(0.000) 1.468 \\
(0.000 W D) 1.350 B \\
(0.809) 1.922\end{array}$ \\
\hline $\begin{array}{l}74.23 \\
74.13 \\
73.87 \\
73.50 \\
70.96\end{array}$ & $\begin{array}{l}25 \\
15 c \\
20 \\
8 \\
15\end{array}$ & $\begin{array}{l}32519.02 \\
32520.04 \\
32522.87 \\
32526.72 \\
32553.69\end{array}$ & $\begin{array}{l}a^{3} \mathrm{D}_{3}-510(3) \\
b{ }^{3} \mathrm{G}_{3}-593(2) \\
a^{1} \mathrm{H}_{5}-567(4) \\
b^{3} \mathrm{G}_{5}-638(4)\end{array}$ & $\begin{array}{l}(0.424 W) 1.316 W \\
(\mathbf{0 . 0 0 0}, 0.284,0.604) \mathbf{0 . 2 5 2}, 0.573,0.846 \\
(0.00) 0.77 \\
(0.000) 1.127\end{array}$ \\
\hline $\begin{array}{l}69.93 \\
68.77 \\
68.45 \\
67.60 \\
65.58\end{array}$ & $\begin{array}{l}8 \\
8 \\
7 \\
5 \\
5\end{array}$ & $\begin{array}{l}32564.55 \\
32576.92 \\
32580.32 \\
32589.29 \\
32610.78\end{array}$ & $b{ }^{1} \mathrm{D}_{2}-558(2)$ & $\begin{array}{l}(0.229, \mathbf{0 . 4 3 3}) 0.947, \mathbf{1 . 1 6 2}, \mathbf{1 . 3 6 8}, 1.554 \\
(0.00) 0.99 \\
(0.000 w) 1.349\end{array}$ \\
\hline
\end{tabular}


TABLE 1. Wavelengths, term combinations, and Zeeman effects of Ta $\mathrm{II}$-Continued

\begin{tabular}{|c|c|c|c|c|}
\hline $\begin{array}{l}\text { Ware } \\
\text { length } \\
\text { 入air }\end{array}$ & $\begin{array}{l}\text { Intensity } \\
\text { and } \\
\text { notes }\end{array}$ & $\begin{array}{l}\text { Wave } \\
\text { number }\end{array}$ & $\begin{array}{l}\text { Term } \\
\text { combination }\end{array}$ & Zeeman pattern \\
\hline 3065.25 & 8 & 32614. 27 & $\left\{\begin{array}{l}a^{1} \mathrm{D}_{2}-461(1) \\
c^{3} \mathrm{~F}_{2}-555(3)\end{array}\right.$ & $(0.000,0.252) \quad 0.869,1.105,1.352$ \\
\hline $\begin{array}{l}\text { 64. } 79 \\
\text { 64. } 17 \\
\text { 64. } 08 \\
\text { 63. } 68\end{array}$ & $\begin{array}{c}125 c \\
6 c \\
8 \\
1\end{array}$ & $\begin{array}{l}32619.20 \\
32625.78 \\
32626.77 \\
32631.60\end{array}$ & $\begin{array}{l}a^{3} \mathrm{H}_{4}-484(4) \\
a^{1} \mathrm{G}_{4}-468(3) \\
b^{3} \mathrm{H}_{5}-580(4) \\
c^{1} \mathrm{D}_{2}-656(3)\end{array}$ & $\begin{array}{l}(0.445) 1.186 \\
(0.00) 1.22 \\
(0.00) 1.35\end{array}$ \\
\hline $\begin{array}{l}63.49 \\
62.52 \\
61.44 \\
60.65 \\
59.63\end{array}$ & $\begin{array}{l}8 \\
35 c \\
20 \\
50 c \\
15\end{array}$ & $\begin{array}{l}\text { 32633. } 02 \\
32643.40 \\
32654.92 \\
\text { 32663. } 26 \\
32674.17\end{array}$ & $\begin{array}{l}a^{5} \mathrm{D}_{0}-452(1) \\
a^{3} \mathrm{D}_{3}-511(2) \\
b^{3} \mathrm{D}_{2}-630(2) \\
a^{3} \mathrm{G}_{3}-444(3) \\
a^{5} \mathrm{D}_{2}-471(3)\end{array}$ & $\begin{array}{l}(0.00) 1.45 \\
(0.000) 1.278 \\
(0.082) 0.755 \\
(0.000 w) 0.910 w \\
(\mathbf{0 . 0 0 0 ,} 0.377,0.751) \quad \mathbf{0 . 3 3 6}, 0.707,1.102,1.487\end{array}$ \\
\hline 58. 73 & 12 & 32683.83 & $a^{5} \mathrm{~F}_{2}-337(2)$ & $(0.000,0.341) 0.537,0.869,1.176,1.482$ \\
\hline 57. 23 & $150 c$ & 32699.88 & $\begin{array}{l}a^{5} \mathrm{D}_{1}-461(1) \\
b^{3} \mathrm{~F}_{3}-472(4)\end{array}$ & $(\mathbf{0 . 0 0 0}, 0.198,0.413,0.656) 0.988,1.176,1.382,1.616, \mathbf{1 . 8 4 9}$ \\
\hline $\begin{array}{l}\text { 56. } 62 \\
56.12 \\
55.48\end{array}$ & $\begin{array}{c}75 c \\
40 \\
2\end{array}$ & $\begin{array}{l}32706.37 \\
32711.68 \\
32718.53\end{array}$ & $\begin{array}{l}a^{5} \mathrm{D}_{4}-499(4) \\
a^{3} \mathrm{H}_{6}-506(6) \\
a^{3} \mathrm{D}_{2}-498(1)\end{array}$ & $\begin{array}{l}(0.167 w) 1.216 w \\
(0.000 w D) 1.153 B \\
(\mathbf{0 . 0 0}, 0.81) \mathbf{0 . 4 6}, 1.32,2.03\end{array}$ \\
\hline $\begin{array}{l}53.10 \\
52.59\end{array}$ & $\begin{array}{r}50 \\
125\end{array}$ & $\begin{array}{l}32744.12 \\
32749.52\end{array}$ & $a^{5} \mathrm{D}_{3}-484(4)$ & $\begin{array}{l}(\mathbf{0 . 0 0 0 , 0 . 1 9 5 , 0 . 3 9 9 , 0 . 6 1 5 )} \mathbf{0 . 8 4 0 , 1 . 0 6 7 , 1 . 2 6 5 , 1 . 4 5 8 , 1 . 6 5 6 , 1 . 8 5 6} \\
(0.000 W D) 0.624 A\end{array}$ \\
\hline 51. 46 & 15 & 32761.63 & $\left\{\begin{array}{l}a^{3} \mathrm{G}_{4}-454(5) \\
a^{1} \mathrm{H}_{5}-569(4)\end{array}\right.$ & $(\mathbf{0 . 0 0 0}, 0.128,0.251,0.392,0.525) \mathbf{0 . 4 5 0}, 0.620,0.739,0.864,1.004$ \\
\hline $\begin{array}{l}45.51 \\
45.37\end{array}$ & $\begin{array}{c}2 \\
25 c\end{array}$ & $\begin{array}{l}32825.68 \\
32827.20\end{array}$ & $\begin{array}{l}b{ }^{3} \mathrm{P}_{2}-513(1) \\
a^{1} \mathrm{D}_{2}-463(2)\end{array}$ & $(0.224,0.440) 0.913,1.112,1.316, \ldots$ \\
\hline $\begin{array}{l}\text { 44. } 13 \\
\text { 42. } 92\end{array}$ & $\begin{array}{l}3 c \\
4\end{array}$ & $\begin{array}{l}32840.55 \\
32853.63\end{array}$ & $a{ }^{5} \mathrm{P}_{1}-435(1)$ & $\begin{array}{ll}(0.98) & 1.50 \\
(0.00) & 1.16\end{array}$ \\
\hline & $100 c$ & & $a^{3} \mathrm{G}_{3}-446(4)$ & $(\mathbf{0 . 0 0 0 ,} 0.336,0.679) 0.232,0.568,0.916,1.256,1.537, \ldots$ \\
\hline 40. 72 & $50 c$ & 32877.44 & ${ }^{3} \mathrm{P}_{2}-385(1)$ & $(0.000,0.878) 0.477,1.326,2.187 w$ \\
\hline $\begin{array}{l}39.85 \\
39.44\end{array}$ & $\begin{array}{r}50 \\
4\end{array}$ & $\begin{array}{l}32886.83 \\
32891.25\end{array}$ & $\begin{array}{l}a^{3} \mathrm{D}_{1}-475(2) \\
b^{3} \mathrm{D}_{1}-628(1)\end{array}$ & $(\mathbf{0 . 0 0 0 , 0 . 4 4 4 )} 0.863,1.272, \mathbf{1 . 7 3 6}$ \\
\hline 37.51 & $100 c$ & 32912. 12 & $\left\{\begin{array}{l}a{ }^{3} \mathrm{~F}_{3}-397(4) \\
a^{5} \mathrm{D}_{1}-463(2)\end{array}\right.$ & $(0.102,0.308, \mathbf{0 . 4 7 0}) \ldots . ?$ \\
\hline $\begin{array}{l}36.61 \\
35.53\end{array}$ & $\begin{array}{l}50 \\
7 c\end{array}$ & $\begin{array}{l}32921.84 \\
32933.57\end{array}$ & $\begin{array}{lll}a & 1 & \mathrm{~F}_{3}-577(2) \\
b & { }^{3} \mathrm{~F}_{3}-475(2)\end{array}$ & $\begin{array}{l}(0.000 w) 0.923 A \\
(\mathbf{0 . 0 0 0 , 0 . 2 8 8 , 0 . 5 9 3 )} \mathbf{0 . 4 2 9}, 0.710\end{array}$ \\
\hline 34.91 & 10 & 32940. 29 & $\left\{\begin{array}{l}b^{3} \mathrm{G}_{4}-611(4) \\
a^{5} \mathrm{D}_{3}-486(2)\end{array}\right.$ & $(\mathbf{0 . 0 0 0 , 0 . 3 2 8 , 0 . 6 2 8 )} 1.471,1.784, \mathbf{2 . 1 0 5}$ \\
\hline $\begin{array}{l}\text { 34. } 01 \\
\text { 33. } 12 \\
\text { 32. } 77 \\
32.34\end{array}$ & $\begin{array}{r}5 c \\
15 c \\
1 \\
6\end{array}$ & $\begin{array}{l}\text { 32950. } 08 \\
\text { 32959. } 78 \\
\text { 32963. } 55 \\
\text { 32968. } 24\end{array}$ & $\begin{array}{l}c{ }^{3} \mathrm{~F}_{2}-558(1) \\
a{ }^{5} \mathrm{P}_{2}-448(3) \\
a^{1} \mathrm{G}_{4}-471(3) \\
a^{3} \mathrm{D}_{1}-475(1)\end{array}$ & $\begin{array}{l}(\mathbf{0 . 0 0 ,} 0.74) \mathbf{0 . 0 0 , 0 . 7 4} \\
(\mathbf{0 . 0 0 0}, 0.183,0.385) \mathbf{0 . 8 9 2}, 1.069,1.246 \\
(0.000) 1.021 \\
(0.652) 0.865,1.507\end{array}$ \\
\hline $\begin{array}{l}\text { 31. } 22 \\
\text { 30. } 68 \\
\text { 29. } 70 \\
\text { 28. } 44 \\
27.62\end{array}$ & $\begin{array}{r}40 c \\
8 \\
2 \\
25 \\
30\end{array}$ & $\begin{array}{l}\text { 32980. } 63 \\
32986.25 \\
32996.98 \\
\text { 33010. } 74 \\
33019.63\end{array}$ & $\begin{array}{l}a{ }^{3} \mathrm{D}_{3}-515(2) \\
b{ }^{3} \mathrm{~F}_{4}-514(4) \\
a^{3} \mathrm{~F}_{2}-361(2) \\
a \\
{ }^{5} \mathrm{P}_{3}-454(2) \\
a^{5} \mathrm{D}_{2}-475(2)\end{array}$ & $\begin{array}{l}(0.000 w) 1.324 A \\
(0.365 w) 1.274 w \\
(0.44) 0.93 D \\
(0.00) 0.84 \\
(0.213, \mathbf{0 . 3 7 0}) 1.105, \mathbf{1 . 2 7 3 , 1 . 4 8 0 , 1 . 6 5 2}\end{array}$ \\
\hline $\begin{array}{l}24.29 \\
23.94 \\
23.20 \\
22.56 \\
21.80\end{array}$ & $\begin{array}{r}2 \\
2 \\
5 \\
50 \\
70\end{array}$ & $\begin{array}{l}\text { 33056. } 02 \\
33059.81 \\
33067.88 \\
33074.90 \\
\text { 33083. } 28\end{array}$ & $\begin{array}{l}b^{1} \mathrm{D}_{2}-563(3) \\
c{ }^{3} \mathrm{~F}_{4}-561(4) \\
a^{3} \mathrm{G}_{3}-448(3) \\
a^{1} \mathrm{G}_{4}-472(4) \\
a^{5} \mathrm{D}_{4}-503(3)\end{array}$ & 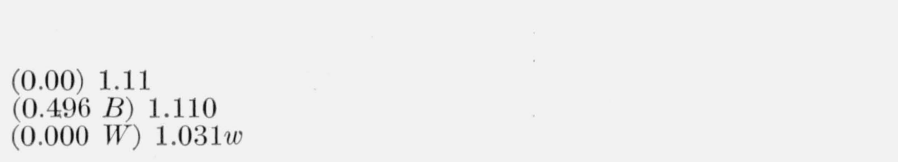 \\
\hline $\begin{array}{l}\text { 21. } 18 \\
\text { 20. } 52 \\
\text { 20. } 17 \\
\text { 19. } 34 \\
\text { 18. } 70\end{array}$ & $\begin{array}{r}4 \\
5 \\
50 \\
8 \\
5\end{array}$ & $\begin{array}{l}\text { 33090. } 04 \\
\text { 33097. } 22 \\
\text { 33101. } 06 \\
\text { 33110. } 22 \\
\text { 33117. } 24\end{array}$ & $\begin{array}{l}c{ }^{3} \mathrm{~F}_{2}-560(2) \\
b{ }^{3} \mathrm{D}_{1}-630(2) \\
a^{5} \mathrm{D}_{2}-475(1) \\
c{ }^{3} \mathrm{P}_{1}-593(2)\end{array}$ & $\begin{array}{l}(0.000) 1.225 w \\
(0.000) 1.460 \\
(\mathbf{0 . 0 0 0 ,} 0.197) \mathbf{0 . 9 1 9 , 1 . 1 5 0 , 1 . 3 6 0}\end{array}$ \\
\hline $\begin{array}{l}\text { 18. } 29 \\
\text { 16. } 07 \\
\text { 15. } 84\end{array}$ & $\begin{array}{r}10 \\
25 \\
4\end{array}$ & $\begin{array}{l}\text { 33121. } 66 \\
33146.10 \\
33148.56\end{array}$ & $\begin{array}{l}d^{3} \mathrm{~F}_{4}-646(3) \\
a^{3} \mathrm{D}_{2}-503(3) \\
c^{1} \mathrm{G}_{4}-682(3)\end{array}$ & $\begin{array}{l}(0.125) 1.086 \\
(0.000 w) 1.363 B \\
(0.00) 1.17\end{array}$ \\
\hline 15. 17 & 10 & 33156. 01 & $\left\{\begin{array}{l}b{ }^{1} \mathrm{D}_{2}-564(1) \\
b \\
{ }^{3} \mathrm{P}_{1}-505(1)\end{array}\right.$ & $(0.000 w) 1.306 w$ \\
\hline 14. 94 & 20 & 33158.45 & $b^{3} \mathrm{H}_{5}-585(5)$ & (0.304) 1.088$\rfloor W$ \\
\hline
\end{tabular}


TABLE 1. Wavelengths, term combinations, and Zeeman effects of Ta II-Continued

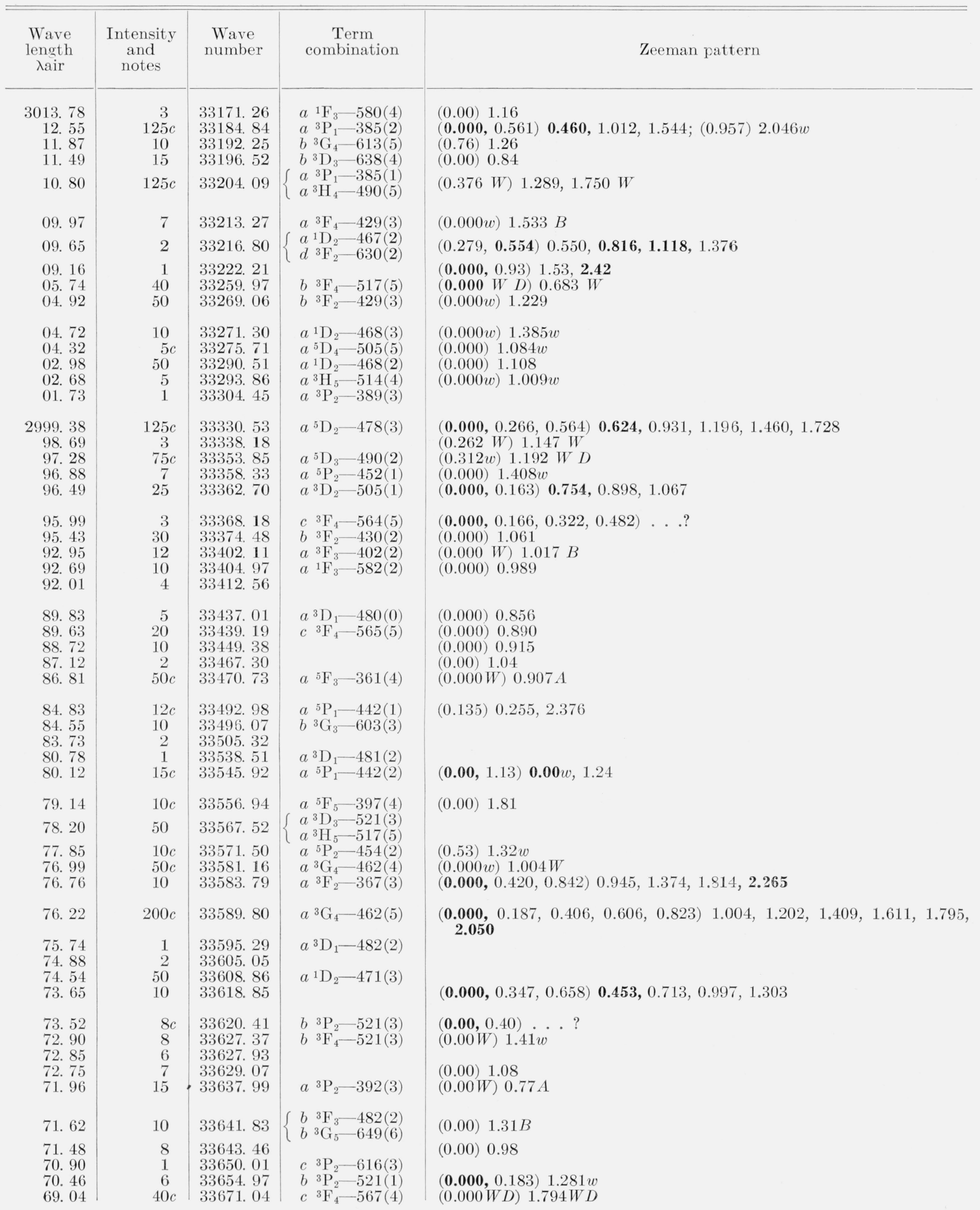


TABLE 1. Wavelengths, term combinations, and Zeeman effects of Ta $\mathrm{II}$-Continued

\begin{tabular}{|c|c|c|c|c|}
\hline $\begin{array}{l}\text { Wave } \\
\text { length } \\
\text { 入air }\end{array}$ & $\begin{array}{l}\text { Intensity } \\
\text { and } \\
\text { notes }\end{array}$ & $\begin{array}{l}\text { Wave } \\
\text { number }\end{array}$ & $\begin{array}{c}\text { Term } \\
\text { combination }\end{array}$ & Zeeman pattern \\
\hline $\begin{array}{r}\text { 2968. } 64 \\
68.29 \\
67.81 \\
66.18 \\
65.93\end{array}$ & $\begin{array}{l}10 \\
75 c \\
5 c \\
3 \\
50 c\end{array}$ & $\begin{array}{l}\text { 33675. } 66 \\
33679.60 \\
33685.01 \\
\text { 33703. } 60 \\
\text { 33706. } 39\end{array}$ & $\begin{array}{l}a^{3} \mathrm{G}_{3}-454(2) \\
a^{3} \mathrm{H}_{4}-495(3) \\
d^{3} \mathrm{~F}_{4}-652(4) \\
a^{5} \mathrm{~F}_{1}-337(1)\end{array}$ & $\begin{array}{l}(\mathbf{0 . 0 0 0}, 0.165,0.345,0.519) \\
(\mathbf{0 . 3 7 0}, 0.522,0.668 \\
(\mathbf{0 . 0 0 0}, 0.195,0.568,0.850) \\
(0.000 w) 1.369 W \\
(0.269) 0.165,0.383\end{array}$ \\
\hline $\begin{array}{l}\text { 65. } 14 \\
64.02 \\
62.20 \\
61.47 \\
60.71\end{array}$ & $\begin{array}{c}250 c \\
50 c \\
2 \\
3 \\
3\end{array}$ & $\begin{array}{l}33715.33 \\
33728.16 \\
33748.84 \\
33757.12 \\
33765.78\end{array}$ & $\begin{array}{l}a{ }^{5} \mathrm{~F}_{1}-337(2) \\
a^{5} \mathrm{D}_{2}-482(2)\end{array}$ & $\begin{array}{l}(\mathbf{0 . 0 0 0}, 0.748) 0.771, \mathbf{1 . 5 5 4 W} \\
(0.000 w) 1.453 \\
(0.00) 1.55 \\
(0.000) 0.669 \\
(\mathbf{0 . 0 0 0}, 0.283,0.571) 1.369,1.695, \ldots\end{array}$ \\
\hline $\begin{array}{l}\text { 60. } 37 \\
\text { 60. } 12 \\
58.61 \\
\text { 58. } 12 \\
57.88\end{array}$ & $\begin{array}{l}7 \\
3 \\
3 c \\
10 \\
50\end{array}$ & $\begin{array}{l}33769.72 \\
33772.53 \\
33789.74 \\
33795.37 \\
\text { 33798. } 09\end{array}$ & $\begin{array}{l}a{ }^{1} \mathrm{P}_{1}-571(1) \\
a^{3} \mathrm{H}_{4}-496(3) \\
a^{3} \mathrm{~F}_{4}-435(3)\end{array}$ & 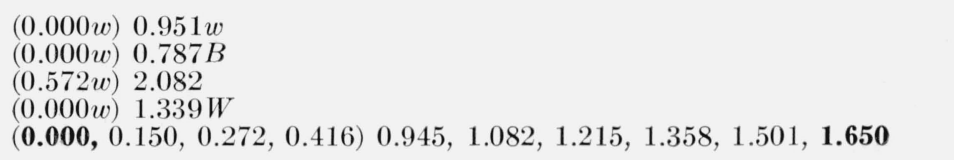 \\
\hline 56.84 & $100 c$ & 33810.07 & $\begin{array}{l}a^{5} \mathrm{D}_{3}-495(3) \\
c^{3} \mathrm{P}_{0}-571(1)\end{array}$ & $(0.171,0.355, \mathbf{0 . 4 9 3}) 0.975,1.141, \mathbf{1 . 3 0 4}, \mathbf{1 . 4 7 3}, 1.637,1.802$ \\
\hline 56.43 & 10 & 33814. 73 & $\left\{\begin{array}{l}a^{3} \mathrm{G}_{5}-466(4) \\
a^{1} \mathrm{H}_{5}-580(4)\end{array}\right.$ & $(0.000 w) 0.894 A$ \\
\hline $\begin{array}{l}55.79 \\
55.32 \\
54.92\end{array}$ & $\begin{array}{c}3 \\
50 \\
8 c\end{array}$ & $\begin{array}{l}33822.04 \\
33827.38 \\
33831.97\end{array}$ & $\begin{array}{l}b{ }^{3} \mathrm{P}_{1}-511(2) \\
b^{3} \mathrm{H}_{6}-598(5)\end{array}$ & $\begin{array}{l}(0.00) 1.31 A \\
(0.000 W D) 1.319 B\end{array}$ \\
\hline $\begin{array}{l}\text { 53. } 97 \\
53.00 \\
\text { 51. } 90 \\
\text { 49. } 92 \\
\text { 49. } 02\end{array}$ & $\begin{array}{l}1 \\
100 \\
75 \\
70 c \\
30\end{array}$ & $\begin{array}{l}33842.84 \\
33853.96 \\
33866.56 \\
33889.26 \\
33899.66\end{array}$ & $\begin{array}{l}a^{5} \mathrm{D}_{4}-510(3) \\
b^{3} \mathrm{~F}_{2}-435(3) \\
a^{5} \mathrm{D}_{3}-495(2) \\
b^{3} \mathrm{~F}_{3}-484(4)\end{array}$ & $\begin{array}{l}(\mathbf{0 . 0 0 0}, 0.150) \ldots ? \\
(0.000 w) 1.116 \\
(0.000 w) 1.372 W D \\
(\mathbf{0 . 0 0 0}, 0.263,0.541,0.884) 0.726,1.003,1.264,1.521,1.834,2.176 \\
(0.000 W D) 1.451\end{array}$ \\
\hline 48. 53 & 10 & 33905. 28 & $\left\{\begin{array}{l}a^{3} \mathrm{D}_{2}-510(3) \\
c^{3} \mathrm{~F}_{4}-569(4)\end{array}\right.$ & $(0.414) 0.903$ \\
\hline $\begin{array}{l}47.21 \\
45.59 \\
44.83\end{array}$ & $\begin{array}{r}4 \\
60 \\
100\end{array}$ & $\begin{array}{l}33920.50 \\
33939.09 \\
33947.89\end{array}$ & $\begin{array}{ll}a^{5} \mathrm{D}_{3}-496(3) \\
a^{3} \mathrm{D}_{3}-524(4)\end{array}$ & 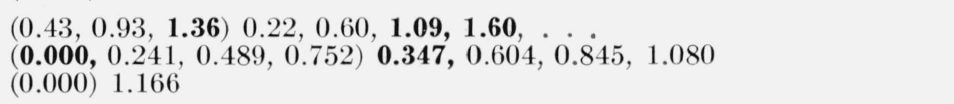 \\
\hline 44.57 & $50 c$ & 33950.86 & $\begin{cases}b & { }^{3} \mathrm{G}_{4}-621(4) \\
b & { }^{3} \mathrm{P}_{1}-513(1)\end{cases}$ & $(0.000) 1.065$ \\
\hline $\begin{array}{l}\text { 44. } 27 \\
\text { 43. } 48 \\
\text { 43. } 09 \\
\text { 38. } 02\end{array}$ & $\begin{array}{l}3 \\
5 c \\
20 \\
75 c\end{array}$ & $\begin{array}{l}\text { 33954. } 40 \\
33963.44 \\
33967.95 \\
34026.60\end{array}$ & $\begin{array}{l}a^{1} \mathrm{D}_{2}-475(2) \\
d^{3} \mathrm{~F}_{4}-654(3) \\
b^{3} \mathrm{G}_{5}-652(4) \\
a^{3} \mathrm{D}_{3}-525(3)\end{array}$ & $\begin{array}{l}(0.40) 1.04 \\
(0.00 w) 1.06 w \\
(0.376 \quad B) 1.174 \quad D \\
(0.216 \quad B) 1.349 D\end{array}$ \\
\hline 37. 80 & 12 & 34029.14 & $\left\{\begin{array}{l}a^{3} \mathrm{D}_{2}-511(2) \\
b^{3} \mathrm{D}_{3}-646(3)\end{array}\right.$ & $(0.179,0.350, \mathbf{0 . 5 4 9}) 0.717,0.897, \mathbf{1 . 0 6 2}, \mathbf{1 . 2 4 0}, 1.417,1.614$ \\
\hline 36.95 & 50 & 34039.00 & $\left\{\begin{array}{l}a^{5} \mathrm{D}_{1}-475(2) \\
a^{3} \mathrm{D}_{1}-486(2)\end{array}\right.$ & $\begin{array}{ll}(\mathbf{0 . 0 0 0}, 0.209) & 1.044,1.296 w \\
(\mathbf{0 . 0 0 0}, 0.222) & 1.434 w\end{array}$ \\
\hline $\begin{array}{l}\text { 36. } 74 \\
\text { 35. } 95 \\
\text { 35. } 62 \\
\text { 32. } 91\end{array}$ & $\begin{array}{l}10 \\
10 \\
10 \\
30 c\end{array}$ & $\begin{array}{l}34041.38 \\
34050.61 \\
34054.42 \\
34085.88\end{array}$ & $\begin{array}{l}b^{3} \mathrm{G}_{3}-608(2) \\
a^{3} \mathrm{~F}_{2}-372(2) \\
b{ }^{3} \mathrm{~F}_{3}-486(2)\end{array}$ & $\begin{array}{l}(\mathbf{0 . 0 0 0}, 0.273,0.546) \mathbf{0 . 2 9 2}, 0.584,0.857,1.123 \\
(0.138, \mathbf{0 . 2 5 7}) 0.475, \mathbf{0 . 6 0 8}, \mathbf{0 . 7 4 5}, 0.872 \\
(0.059) 1.023 \\
(\mathbf{0 . 0 0 0 ,} 0.175,0.337) \mathbf{0 . 6 0 7}, 0.786,0.954,1.104,1.285,1.419\end{array}$ \\
\hline $\begin{array}{l}31.69 \\
29.93\end{array}$ & $\begin{array}{r}5 \\
20\end{array}$ & $\begin{array}{l}34100.00 \\
34120.55\end{array}$ & $a^{5} \mathrm{D}_{1}-475(1)$ & $\begin{array}{lll}(0.000, & 0.094) & 0.439,0.532 \\
(0.000) & 1.506 & \end{array}$ \\
\hline 29. 45 & 15 & 34126.14 & $\left\{\begin{array}{l}d^{3} \mathrm{~F}_{4}-656(3) \\
a^{3} \mathrm{G}_{4}-468(3)\end{array}\right.$ & $(\mathbf{0 . 0 0 0}, 0.203,0.376,0.560) \mathbf{0 . 4 6 7}, 0.643,0.844$ \\
\hline $\begin{array}{l}\text { 28. } 94 \\
\text { 28. } 29\end{array}$ & $\begin{array}{l}2 \\
3\end{array}$ & $\begin{array}{l}34132.06 \\
34139.68\end{array}$ & $\begin{array}{l}c{ }^{3} \mathrm{~F}_{2}-570(3) \\
b{ }^{3} \mathrm{H}_{4}-585(5)\end{array}$ & \\
\hline $\begin{array}{l}27.51 \\
26.72 \\
26.60\end{array}$ & $\begin{array}{l}15 \\
20 c \\
7\end{array}$ & $\begin{array}{l}34148.72 \\
34158.00 \\
34159.35\end{array}$ & $\begin{array}{l}a^{3} \mathrm{D}_{1}-487(1) \\
a^{3} \mathrm{D}_{2}-513(1) \\
b{ }^{3} \mathrm{P}_{1}-515(2)\end{array}$ & $\begin{array}{l}(0.107) 0.798 \\
(\mathbf{0 . 0 0 0}, 0.184) \quad \mathbf{1 . 0 7 7}, 1.251,1.422 \\
(0.00) 1.32\end{array}$ \\
\hline 25. 55 & 8 & $\begin{array}{l}34171.63 \\
34203.12\end{array}$ & $\left\{\begin{array}{l}c{ }^{3} \mathrm{~F}_{3}-577(2) \\
a^{5} \mathrm{D}_{2}-486(2)\end{array}\right.$ & $(0.341,0.644) 0.835,1.126,1.492,1.781$ \\
\hline 22.85 & $50 c$ & 34203.12 & & $(0.000 w) 0.885 \mathrm{D}$ \\
\hline $\begin{array}{l}\text { 22. } 28 \\
\text { 22. } 13 \\
\text { 21. } 44 \\
\text { 18. } 96 \\
\text { 18. } 09\end{array}$ & $\begin{array}{c}30 c \\
40 \\
8 \\
200 c \\
12\end{array}$ & $\begin{array}{l}\text { 34209. } 78 \\
\text { 34211. } 39 \\
34219.66 \\
\text { 34248. } 75 \\
\text { 34258. } 92\end{array}$ & $\begin{array}{l}a{ }^{5} \mathrm{P}_{3}-466(4) \\
a^{5} \mathrm{D}_{3}-499(4) \\
a^{5} \mathrm{D}_{4}-514(4) \\
a^{3} \mathrm{~F}_{4}-440(4)\end{array}$ & $\begin{array}{l}(0.000,0.248,0.494,0.747) 0.449,0.694,0.955,1.202,1.436,1.692,1.968 \\
(0.000) 0.818 \\
(0.239 w) 1.131 W \\
(0.402 B) 1.168 D\end{array}$ \\
\hline
\end{tabular}


TABLE 1. Wavelengths, term combinations, and Zeeman effects of Ta II-Continued

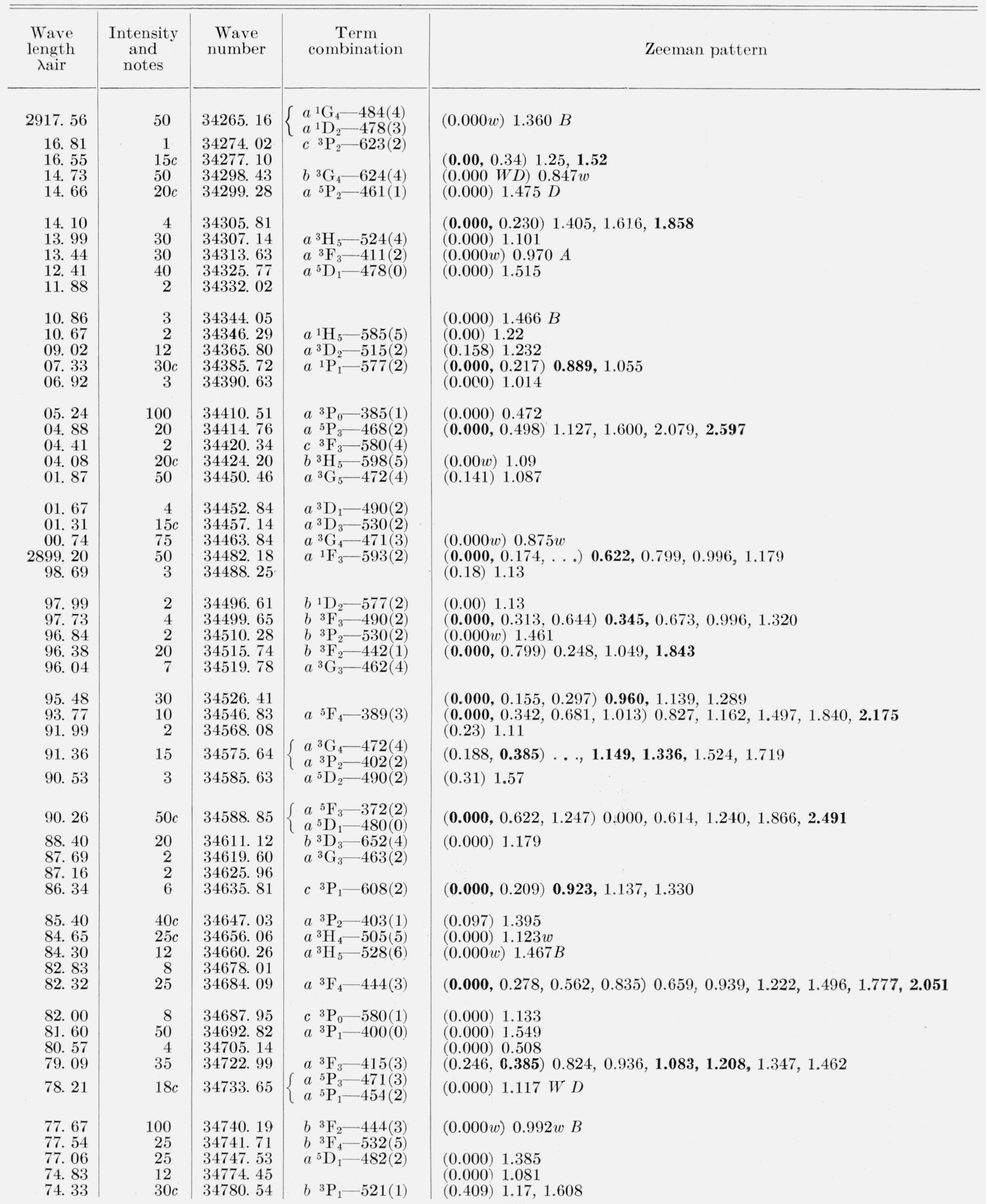


TABLE 1. Wavelengths, term combinations, and Zeeman effects of Ta II-Continued

\begin{tabular}{|c|c|c|c|c|}
\hline $\begin{array}{l}\text { Wave } \\
\text { length } \\
\lambda \text { air }\end{array}$ & $\begin{array}{l}\text { Intensity } \\
\text { and } \\
\text { notes }\end{array}$ & $\begin{array}{l}\text { Wave } \\
\text { number }\end{array}$ & $\begin{array}{c}\text { Term } \\
\text { combination }\end{array}$ & Zeeman pattern \\
\hline 2873. 56 & 40 & 34789.82 & \multirow{4}{*}{$\begin{array}{c}a^{3} \mathrm{D}_{3}-533(4) \\
\left\{\begin{array}{l}d^{3} \mathrm{~F}_{2}-646(3) \\
c^{3} \mathrm{P}_{2}-628(1)\end{array}\right.\end{array}$} & $(0.00 w) 1.64$ \\
\hline 72. 16 & 1 & 34806. 79 & & \\
\hline 71. 89 & 1 & 34810. 06 & & \\
\hline $\begin{array}{l}71.59 \\
70.87\end{array}$ & $\begin{array}{l}5 \\
6\end{array}$ & $\begin{array}{l}34813.72 \\
34822.47\end{array}$ & & $\begin{array}{l}(0.00) 1.20 \\
(0.000) \\
0.956\end{array}$ \\
\hline 69. 51 & $15 c$ & 34838. 98 & \multirow{4}{*}{$\begin{array}{l}a{ }^{3} \mathrm{~F}_{4}-445(5) \\
a{ }^{5} \mathrm{P}_{3}-472(4) \\
b{ }^{3} \mathrm{~F}_{4}-533(4) \\
a^{1} \mathrm{G}_{4}-490(5) \\
b^{1} \mathrm{G}_{4}-602(3)\end{array}$} & $(0.000 W) 1.399 w$ \\
\hline 69. 02 & 6 & 34844.93 & & $(\mathbf{0 . 0 0 0}, 0.396) \mathbf{0 . 7 7 5}, 1.197,1.602$ \\
\hline 68. 64 & 25 & 34849.53 & & $(0.392 B) 1.132 D$ \\
\hline $\begin{array}{l}\text { 68. } 14 \\
67.83\end{array}$ & 20 & $\begin{array}{l}34855.52 \\
34859.39\end{array}$ & & $\begin{array}{l}(0.000 w) 0.893 B \\
(\mathbf{0 . 0 0 0}, 0.176,0.369,0.539) \ldots . ?\end{array}$ \\
\hline 67. 41 & $150 c$ & 34864. 40 & \multirow{5}{*}{$\begin{array}{l}a^{3} \mathrm{H}_{6}-528(6) \\
b^{3} \mathrm{D}_{3}-654(3) \\
a^{3} \mathrm{G}_{3}-466(4) \\
a^{5} \mathrm{~F}_{4}-392(3) \\
a^{3} \mathrm{~F}_{4}-446(4)\end{array}$} & $(0.000 \mathrm{~W}) 1.163 \mathrm{w}$ \\
\hline 66. 85 & 7 & 34871. 20 & & $(0.00) 1.42$ \\
\hline 66. 26 & $18 c$ & 34878.47 & & $(\mathbf{0 . 0 0 0}, 0.280,0.586) 1.223,1.499,1.789, \mathbf{2 . 1 0 2}$ \\
\hline 66. 14 & $35 c$ & 34879.84 & & $(0.000 w) 1.282 w$ \\
\hline 65. 70 & 50 & 34885. 23 & & $(0.000 w) 1.035 A$ \\
\hline 65. 32 & 85 & 34889.85 & \multirow{5}{*}{$\begin{array}{l}a{ }^{5} \mathrm{D}_{4}-521(3) \\
a{ }^{3} \mathrm{P}_{1}-402(2) \\
a^{3} \mathrm{D}_{3}-534(3)\end{array}$} & $(0.000 w) 1.328 \mathrm{~W}$ \\
\hline 64. 26 & 7 & 34902.82 & & $(\mathbf{0 . 0 0 0}, 0.395) \mathbf{0 . 7 5 1}, 1.159,1.571$ \\
\hline 63. 54 & 10 & 34911.56 & & $(0.00 w) 1.24$ \\
\hline 63. 44 & 20 & 34912.80 & & $(0.264 B) 1.105 D$ \\
\hline 62.52 & 12 & 34923. 96 & & $(0.000) 1.112$ \\
\hline 61. 24 & 10 & 34939. 65 & \multirow{5}{*}{ 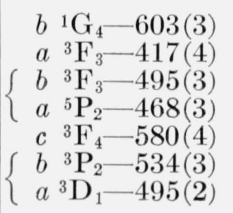 } & $(0.00) 1.01$ \\
\hline 60.88 & 75 & 34943. 99 & & $(\mathbf{0 . 0 0 0}, 0.172,0.358,0.542) \ldots, 0.901,1.075,1.259,1.436,1.637, \mathbf{1 . 8 2 9}$ \\
\hline 59. 94 & 8 & 34955.55 & & $(\mathbf{0 . 0 0 0}, 0.221,0.453) \mathbf{0 . 7 6 6}, 0.984$ \\
\hline 59. 72 & 12 & 34958. 17 & & $(0.059) 1.056$ \\
\hline 59. 14 & $20 c$ & 34965. 28 & & $\begin{array}{ll}(0.000) & 0.755 \\
(0.000) & 2.305\end{array}$ \\
\hline 58. 75 & 20 & 34970.05 & & \\
\hline 58. 43 & 125 & 34973. 99 & $a^{3} \mathrm{P}_{1}-403(1)$ & $(0.317) 1.223,1.556$ \\
\hline 57. 34 & 8 & 34987. 33 & $a^{3} \mathrm{D}_{2}-52 \mathrm{i}(1)$ & $(\mathbf{0 . 0 0 0}, 0.399) \mathbf{0 . 8 5 3}, 1.227$ \\
\hline 56.68 & 50 & 34995.40 & $a^{5} \mathrm{D}_{0}-475(1)$ & $(0.000) 1.502$ \\
\hline & & 35005.55 & & $(0.00) 1.21$ \\
\hline 55. 34 & $20 c$ & 35011. 75 & $b^{3} \mathrm{~F}_{3}-495(2)$ & $(\mathbf{0 . 0 0 0}, 0.587,1.155,1.678) \mathbf{0 . 4 4 1}, 0.984,1.581$ \\
\hline 55. 18 & 5 & 35013.75 & $d^{3} \mathrm{~F}_{4}-665(4)$ & $(0.00) 1.10$ \\
\hline $\begin{array}{l}\text { 54. } 79 \\
54.21\end{array}$ & $\begin{array}{l}6 \\
2\end{array}$ & $\begin{array}{l}35018.60 \\
35025.68\end{array}$ & & $\begin{array}{l}(0.000) 0.846 \\
(0.00) 0.90 A\end{array}$ \\
\hline 53.54 & 8 & 35033.85 & $b{ }^{3} \mathrm{D}_{3}-656(3)$ & $(0.264,0.504, \mathbf{0 . 7 5 3}) 0.501,0.766, \mathbf{1 . 0 3 2}, \mathbf{1 . 2 7 4}, 1.492,1.754$ \\
\hline 53. 18 & 10 & 35038. 26 & $b^{3} \mathrm{P}_{0}-513(1)$ & $(0.00) 1.046$ \\
\hline 52. 94 & 10 & 35041. 32 & $a^{5} \mathrm{D}_{2}-495(3)$ & $(\mathbf{0 . 0 0 0}, 0.161,0.335) \quad \mathbf{0 . 9 8 9}, 1.120,1.288,1.466$ \\
\hline 52. 34 & 150 & 35048.68 & $a^{3} \mathrm{H}_{5}-532(5)$ & $(0.274 B) 1.109 W D$ \\
\hline 50. 98 & 40 & 35065.40 & $b^{3} \mathrm{~F}_{3}-496(3)$ & $(0.389 B) 1.239 W D$ \\
\hline 49. 92 & 10 & 35078.40 & d ${ }^{3} \mathrm{~F}_{4}-66 \mathrm{~b}(5)$ & \\
\hline 49. 86 & 12 & 35079.08 & $a^{5} \mathrm{P}_{3}-475(2)$ & $(\mathbf{0 . 0 0 0}, 0.325,0.654) 0.967,1.297, \mathbf{1 . 6 0 4} \ldots$ ? \\
\hline 49. 51 & 1 & 35083. 43 & $a^{3} \mathrm{G}_{3}-468(2)$ & \\
\hline 49. 06 & 8 & 35089.02 & $a^{3} \mathrm{~F}_{4}-448(3)$ & $(0.000) 1.172$ \\
\hline $\begin{array}{l}\text { 48. } 89 \\
\text { 48. } 33\end{array}$ & $\begin{array}{l}20 c \\
15\end{array}$ & $\begin{array}{l}35091.13 \\
35097.98\end{array}$ & $\begin{array}{l}a^{3} \mathrm{D}_{3}-536(2) \\
a^{5} \mathrm{D}_{2}-495(2)\end{array}$ & $\begin{array}{l}(0.000 w) 1.350 w \\
(0.197) 1.513 D\end{array}$ \\
\hline 47. 65 & 20 & 35106.31 & $a^{1} \mathrm{D}_{2}-486(2)$ & $(0.000) 1.114$ \\
\hline 46.54 & $5 c$ & 35120.05 & $a^{3} \mathrm{G}_{4}-478(3)$ & (0.000) 1.114 \\
\hline 44. 60 & 10 & 35144.00 & $b^{3}{ }^{3} \mathrm{P}_{2}-536(2)$ & \\
\hline 44. 46 & 200 & 35145.75 & $a{ }^{5} \mathrm{~F}_{2}-361(2)$ & $(0.139 B) 0.988 w$ \\
\hline 43. 98 & 4 & 35151.68 & $a{ }^{5} \mathrm{D}_{2}-496(3)$ & \\
\hline 43. 51 & $110 c$ & 35157.50 & $a^{3} \mathrm{H}_{5}-533(4)$ & $(0.000) 1.083$ \\
\hline 41. 61 & 8 & 35180.95 & & $(0.000) 1.112$ \\
\hline 40. 79 & $\underset{75}{8}$ & 35191.13 & $a^{5} \mathrm{D}_{1}-486(2)$ & $(\mathbf{0 . 0 0 0}, 0.368) \quad \mathbf{0 . 7 7 0}, 1.143$ \\
\hline $\begin{array}{l}\text { 40. } 39 \\
39.91\end{array}$ & $\begin{array}{l}75 \\
10\end{array}$ & 35196.13 & & $(0.000) 1.038$ \\
\hline & & 35202. 01 & & $(0.000 w) 0.889 w$ \\
\hline
\end{tabular}


TABLE 1. Wavelengths, term combinations, and Zeeman effects of Ta $\mathrm{II}$-Continued

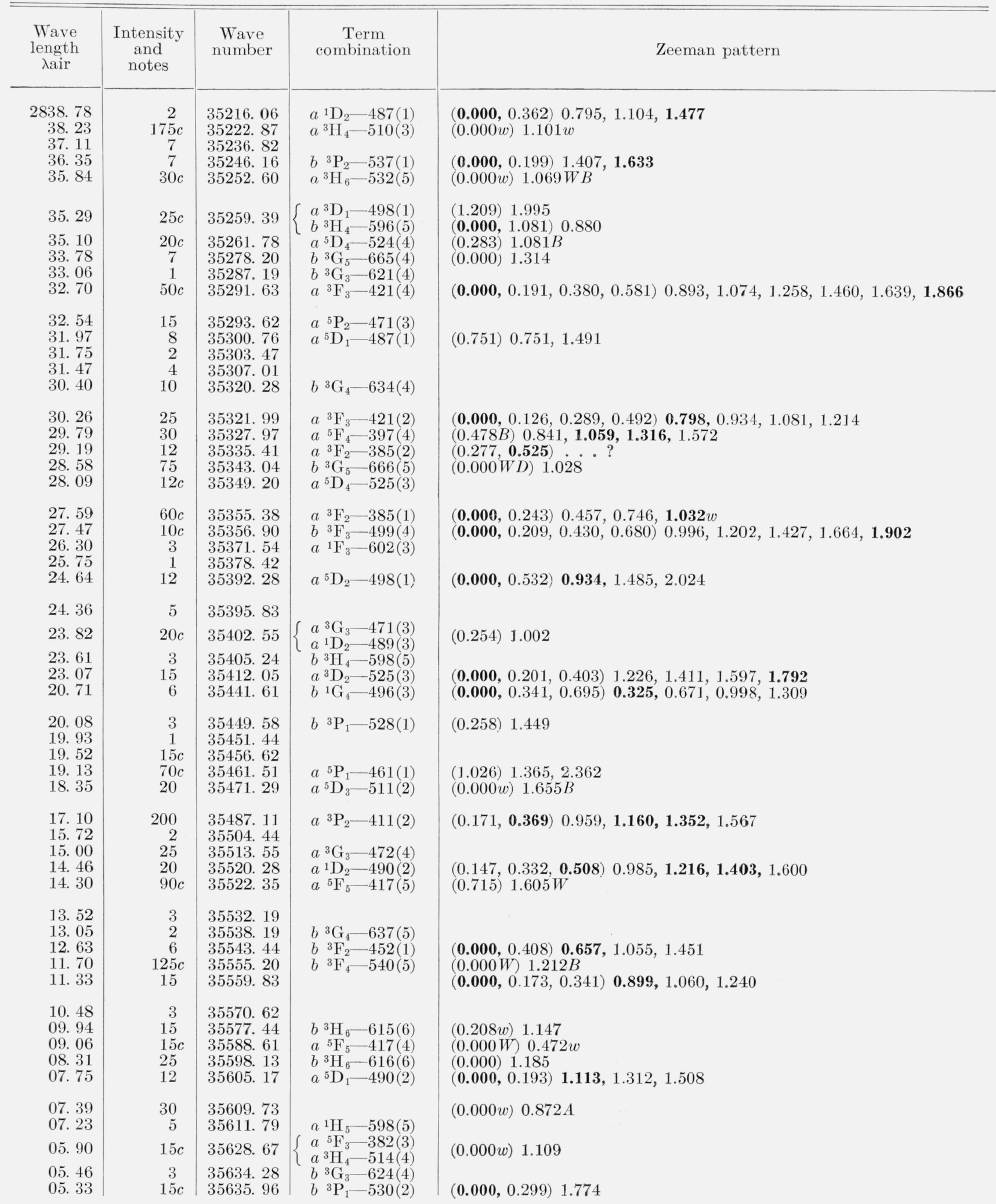


TABLE 1. Wavelengths, term combinations, and Zeeman effects of Ta $\mathrm{II}$-Continued

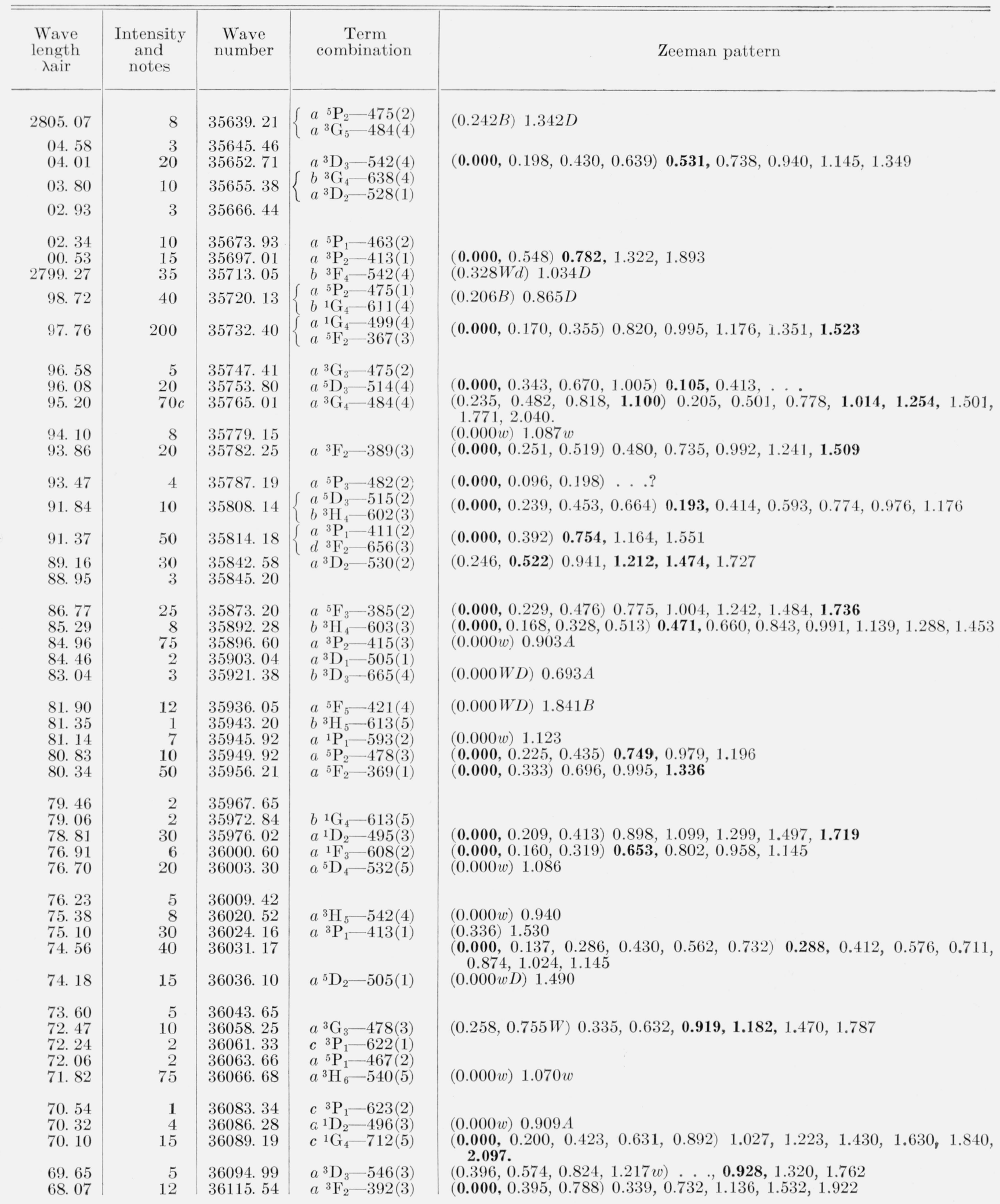


TABLE 1. Wavelengths, term combinations, and Zeeman effects of Ta $\mathrm{II}-$ Continued

\begin{tabular}{|c|c|c|c|c|}
\hline $\begin{array}{l}\text { Wave } \\
\text { length } \\
\text { 入air }\end{array}$ & $\begin{array}{l}\text { Intensity } \\
\text { and } \\
\text { notes }\end{array}$ & $\begin{array}{l}\text { Wave } \\
\text { number }\end{array}$ & $\begin{array}{l}\text { Term } \\
\text { combination }\end{array}$ & Zeeman pattern \\
\hline $\begin{array}{r}2767.92 \\
67.10 \\
66.79 \\
66.40 \\
65.59\end{array}$ & $\begin{array}{r}2 \\
15 \\
1 \\
12 \\
2\end{array}$ & $\begin{array}{l}\text { 36117. } 51 \\
36128.20 \\
36132.27 \\
36137.39 \\
36147.95\end{array}$ & $\begin{array}{l}a^{5} \mathrm{D}_{1}-495(2) \\
a^{3} \mathrm{~F}_{3}-429(3) \\
a^{3} \mathrm{D}_{2}-533(2) \\
a^{5} \mathrm{P}_{1}-468(2) \\
b{ }^{3} \mathrm{P}_{2}-546(3)\end{array}$ & $\begin{array}{l}(0.132) 1.083 \\
(\mathbf{0 . 0 0 0}, 1.286 w)-\mathbf{0 . 1 9 7},+1.091,2.357 \\
(\mathbf{0 . 0 0 0}, 0.515,1.048) \mathbf{0 . 4 2 9}, 0.929,1.437\end{array}$ \\
\hline $\begin{array}{l}\text { 65. } 04 \\
\text { 63. } 36\end{array}$ & $\begin{array}{r}2 \\
30\end{array}$ & $\begin{array}{l}\text { 36155. } 19 \\
36177.16\end{array}$ & $\begin{array}{l}b^{3} \quad \mathrm{~F}_{4}-546(3) \\
a^{5} \mathrm{~F}_{1}-361(2)\end{array}$ & $\begin{array}{l}(\mathbf{0 . 0 0 0}, 0.295,0.600,0.907) 1.224,1.525,1.833,2.124 \\
(\mathbf{0 . 0 0 0}, 0.934) 0.000,0.934, \mathbf{1 . 8 6 6}\end{array}$ \\
\hline 62.05 & 75 & 36194. 27 & $\left\{\begin{array}{l}b^{3} \mathrm{H}_{6}-622(5) \\
b^{3} \mathrm{H}_{5}-616(6)\end{array}\right.$ & $(0.000 W) 1.464$ \\
\hline $\begin{array}{l}\text { 61. } 75 \\
59.28\end{array}$ & $\begin{array}{l}75 \\
20\end{array}$ & $\begin{array}{l}36199.54 \\
36230.59\end{array}$ & $\begin{array}{l}a^{5} \mathrm{~F}_{2}-372(2) \\
a^{5} \mathrm{P}_{3}-486(2)\end{array}$ & $\begin{array}{l}(0.384, \mathbf{0 . 7 7 0}) \\
(\mathbf{0 . 0 0 0}, 0.473,0.990) \\
0.575,1.130,1.610,2.067, \mathbf{2 . 5 3 8}\end{array}$ \\
\hline $\begin{array}{l}\text { 59. } 05 \\
58.16\end{array}$ & $\begin{array}{r}25 \\
2\end{array}$ & $\begin{array}{l}36233.65 \\
36245.36\end{array}$ & $a^{3} \mathrm{~F}_{3}-430(2)$ & $(0.000) 1.097$ \\
\hline 57. 26 & 40 & 36257. 20 & $a^{3} \mathrm{G}_{4}-489(3)$ & $(\mathbf{0 . 0 0 0}, 0.259,0.549,0.871) \mathbf{0 . 1 4 9}, 0.455,0.774,1.015,1.269,1.535, \ldots$ \\
\hline 56. 30 & 25 & 36269. 75 & $\left\{\begin{array}{l}b{ }^{3} \mathrm{P}_{1}-536(2) \\
a^{3} \mathrm{H}_{4}-521(3)\end{array}\right.$ & $(0.000 w) 1.149 A$ \\
\hline 54. 21 & 2 & 36297. 27 & ${ }^{3}{ }^{3} \mathrm{D}_{2}-534(3)$ & $(0.000) 1.228$ \\
\hline $\begin{array}{l}53.46 \\
52.49 \\
51.98 \\
50.35 \\
48.58\end{array}$ & $\begin{array}{r}3 \\
100 \\
3 \\
100 \\
20\end{array}$ & $\begin{array}{l}36307.20 \\
36319.96 \\
36326.79 \\
36348.24 \\
36371.70\end{array}$ & $\begin{array}{l}a{ }^{5} \mathrm{~F}_{3}-389(3) \\
a^{1} \mathrm{D}_{2}-498(1) \\
a{ }^{5} \mathrm{P}_{2}-482(2) \\
b \\
{ }^{3} \mathrm{P}_{1}-537(1)\end{array}$ & $\begin{array}{l}(0.240,0.475, \mathbf{0 . 7 6 2}) 0.486,0.744, \mathbf{0 . 9 9 7}, \mathbf{1 . 2 3 9}, 1.490,1.768 \\
(0.00) 0.93 \\
(0.176) 1.125 \mathrm{~W} \\
(0.098) 1.217\end{array}$ \\
\hline $\begin{array}{l}\text { 46. } 83 \\
\text { 45. } 57 \\
\text { 43. } 98 \\
42.23 \\
40.69\end{array}$ & $\begin{array}{r}75 \\
3 \\
1 \\
15 \\
30\end{array}$ & $\begin{array}{l}36394.81 \\
36411.50 \\
36432.64 \\
36455.83 \\
36476.34\end{array}$ & $\begin{array}{l}a^{5} \mathrm{D}_{3}-521(3) \\
a^{5} \mathrm{D}_{1}-498(1) \\
a^{3} \mathrm{G}_{3}-482(2) \\
a^{3} \mathrm{D}_{2}-536(2)\end{array}$ & 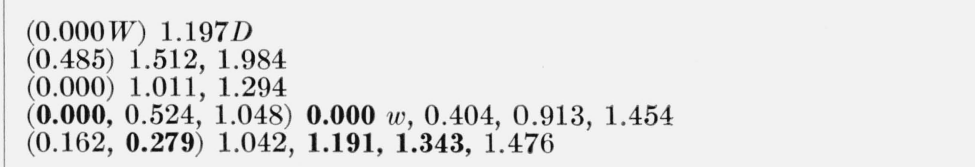 \\
\hline $\begin{array}{l}\text { 40. } 11 \\
\text { 39. } 26 \\
\text { 38. } 20 \\
\text { 37. } 20 \\
\text { 36. } 91\end{array}$ & $\begin{array}{r}25 \\
75 \\
1 \\
2 \\
15\end{array}$ & $\begin{array}{l}\text { 36484. } 09 \\
36495.42 \\
36509.61 \\
36522.89 \\
\text { 36526. } 69\end{array}$ & $\begin{array}{ll}b & { }^{3} \mathrm{~F}_{2}-461(1) \\
a & { }^{3} \mathrm{P}_{2}-421(2)\end{array}$ & $\begin{array}{l}(0.00) 1.22 \\
(0.175, \mathbf{0 . 3 1 0}) 1.045,1.184,1.371,1.528\end{array}$ \\
\hline $\begin{array}{l}\text { 35. } 26 \\
\text { 33. } 67 \\
\text { 33. } 33 \\
\text { 31. } 26 \\
\text { 30. } 98\end{array}$ & $\begin{array}{r}50 \\
2 \\
25 \\
8 \\
2\end{array}$ & $\begin{array}{l}36548.83 \\
36569.98 \\
36574.54 \\
36602.32 \\
36606.11\end{array}$ & $\begin{array}{l}a^{3} \mathrm{~F}_{4}-462(5) \\
a^{3} \mathrm{D}_{1}-511(2) \\
a^{3} \mathrm{D}_{3}-551(3)\end{array}$ & $\begin{array}{l}(0.260 B) 1.257 D \\
(0.000) 0.930\end{array}$ \\
\hline 30. 74 & 50 & 36609.30 & $\left\{\begin{array}{ccc}c & 3 & \mathrm{P}_{2}-646(3) \\
c & 3 \mathrm{~F}-596(5)\end{array}\right.$ & $(0.000 W) 1.151 B$ \\
\hline $\begin{array}{l}\text { 30. } 25 \\
\text { 29. } 93 \\
29.37 \\
28.79\end{array}$ & $\begin{array}{r}1 \\
3 \\
1 \\
15\end{array}$ & $\begin{array}{l}36615.86 \\
36620.19 \\
36627.69 \\
36635.41\end{array}$ & $\begin{array}{ll}c & \\
b & { }^{3} \mathrm{~F}_{3}-511(2) \\
c & { }^{3} \mathrm{P}_{1}-628(1) \\
b & { }^{3} \mathrm{P}_{2}-551(3)\end{array}$ & $\begin{array}{l}(0.424) 0.911,1.340 \\
(0.000) 1.176\end{array}$ \\
\hline $\begin{array}{l}\text { 28. } 32 \\
27.44 \\
\text { 26. } 85 \\
\text { 26. } 41 \\
\text { 26. } 01\end{array}$ & $\begin{array}{r}2 \\
75 \\
4 \\
2 \\
15\end{array}$ & $\begin{array}{l}36641.78 \\
36653.62 \\
36661.47 \\
36667.42 \\
36672.80\end{array}$ & $\begin{array}{l}a^{3} \mathrm{H}_{4}-524(4) \\
a^{5} \mathrm{~F}_{3}-392(3)\end{array}$ & $(0.309) 1.198$ \\
\hline $\begin{array}{l}25.44 \\
24.09 \\
23.85 \\
23.67 \\
23.49\end{array}$ & $\begin{array}{r}75 \\
12 \\
40 \\
1 \\
2\end{array}$ & $\begin{array}{l}36680.49 \\
36698.69 \\
36701.83 \\
36704.30 \\
36706.70\end{array}$ & $\begin{array}{l}a^{3} \mathrm{D}_{1}-513(1) \\
b^{3} \mathrm{H}_{5}-621(4) \\
c^{3} \mathrm{~F}_{3}-603(3)\end{array}$ & $\begin{array}{l}(0.000 \mathrm{~W}) 1.032 D \\
(0.207) 0.841,1.000 \\
(0.00) 0.98 \\
(0.000) 0.845\end{array}$ \\
\hline $\begin{array}{l}22.20 \\
21.82 \\
21.16 \\
20.05 \\
\text { 19. } 89 \\
19.28\end{array}$ & $\begin{array}{r}1 \\
3 \\
1 \\
10 \\
2 \\
1\end{array}$ & $\begin{array}{l}36724.09 \\
36729.22 \\
36738.12 \\
36753.13 \\
36755.26 \\
36763.60\end{array}$ & $a^{3} \mathrm{H}_{4}-525(3)$ & (0.113) 1.043 \\
\hline
\end{tabular}


TABLE 1. Wavelengths, term combinations, and Zeeman effects of Ta II-Continued

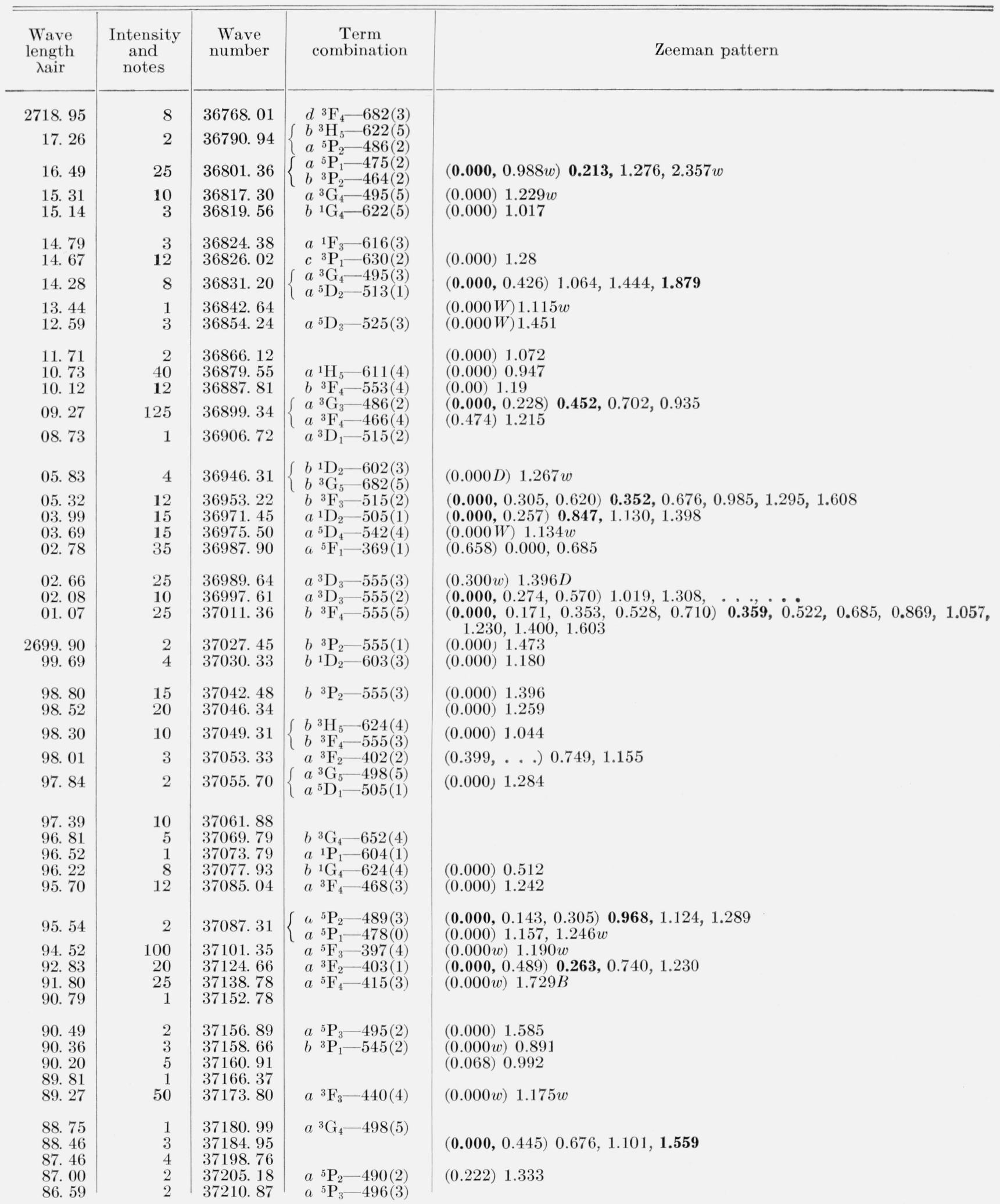


TABLE 1. Wavelengths, term combinations, and Zeeman effects of Ta II-Continued

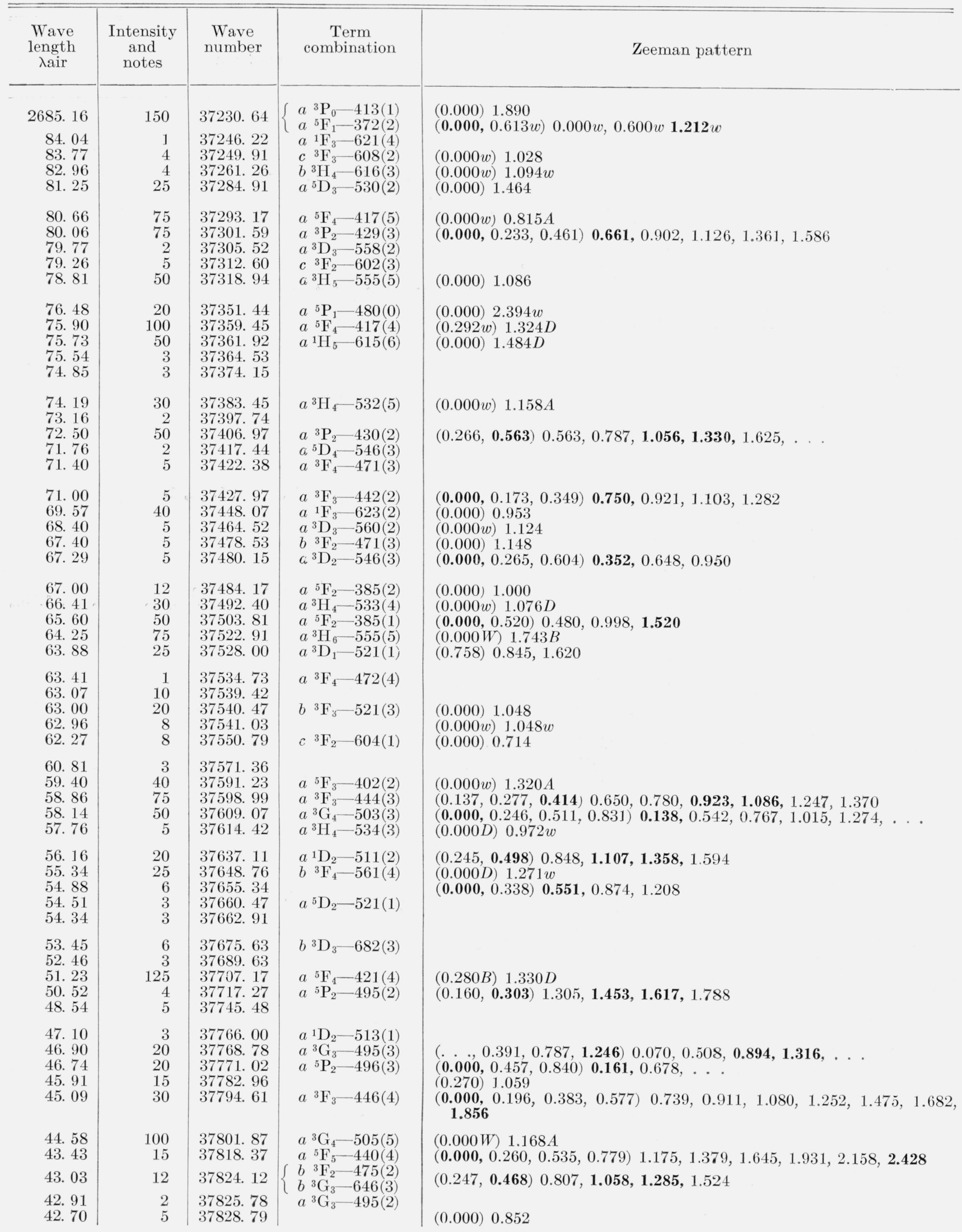


TABLE 1. Wavelengths, term combinations, and Zeeman effects of Ta II-Continued

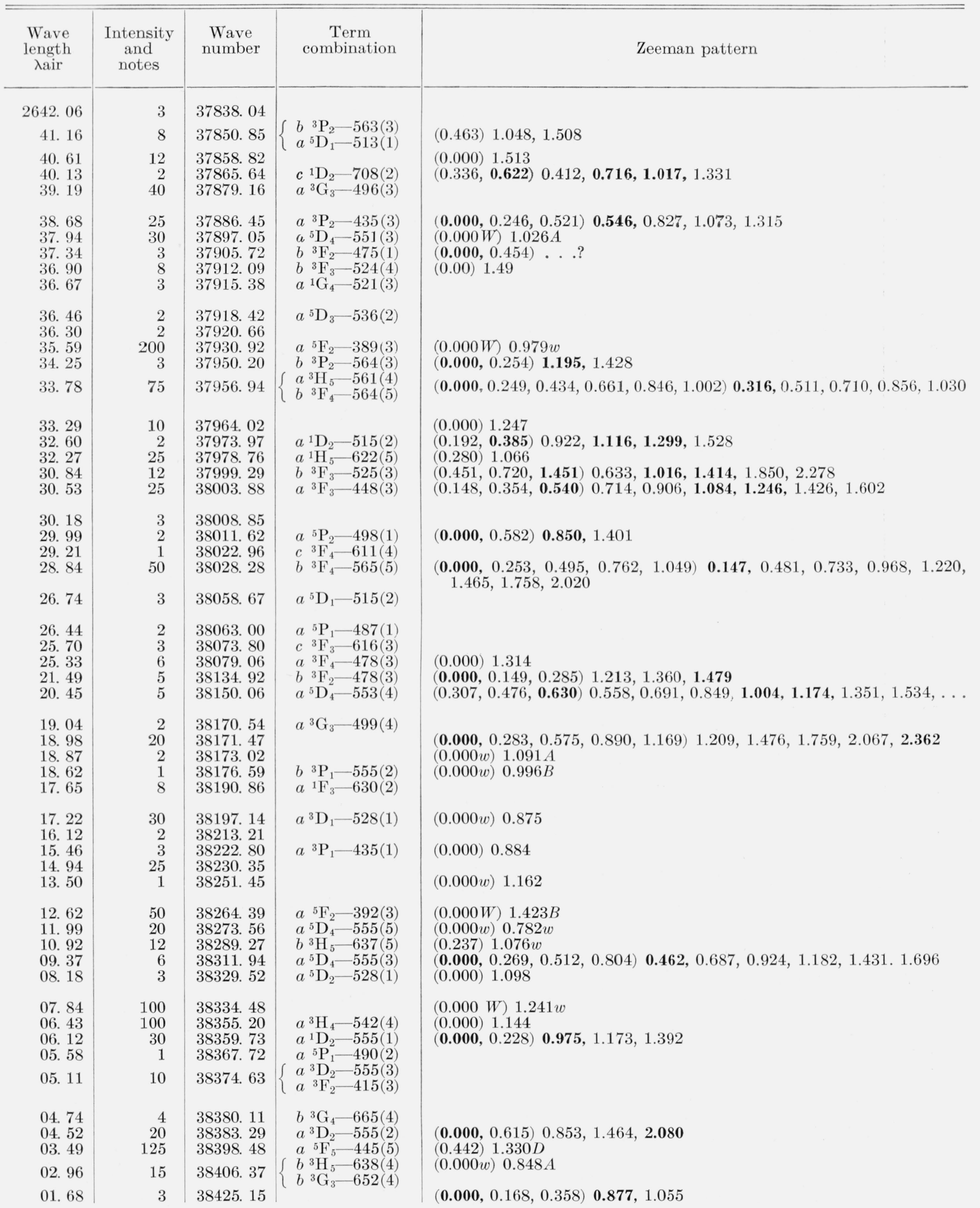


TABLE 1. Wavelengths, term combinations, and Zeeman effects of Ta $\mathrm{II}$-Continued

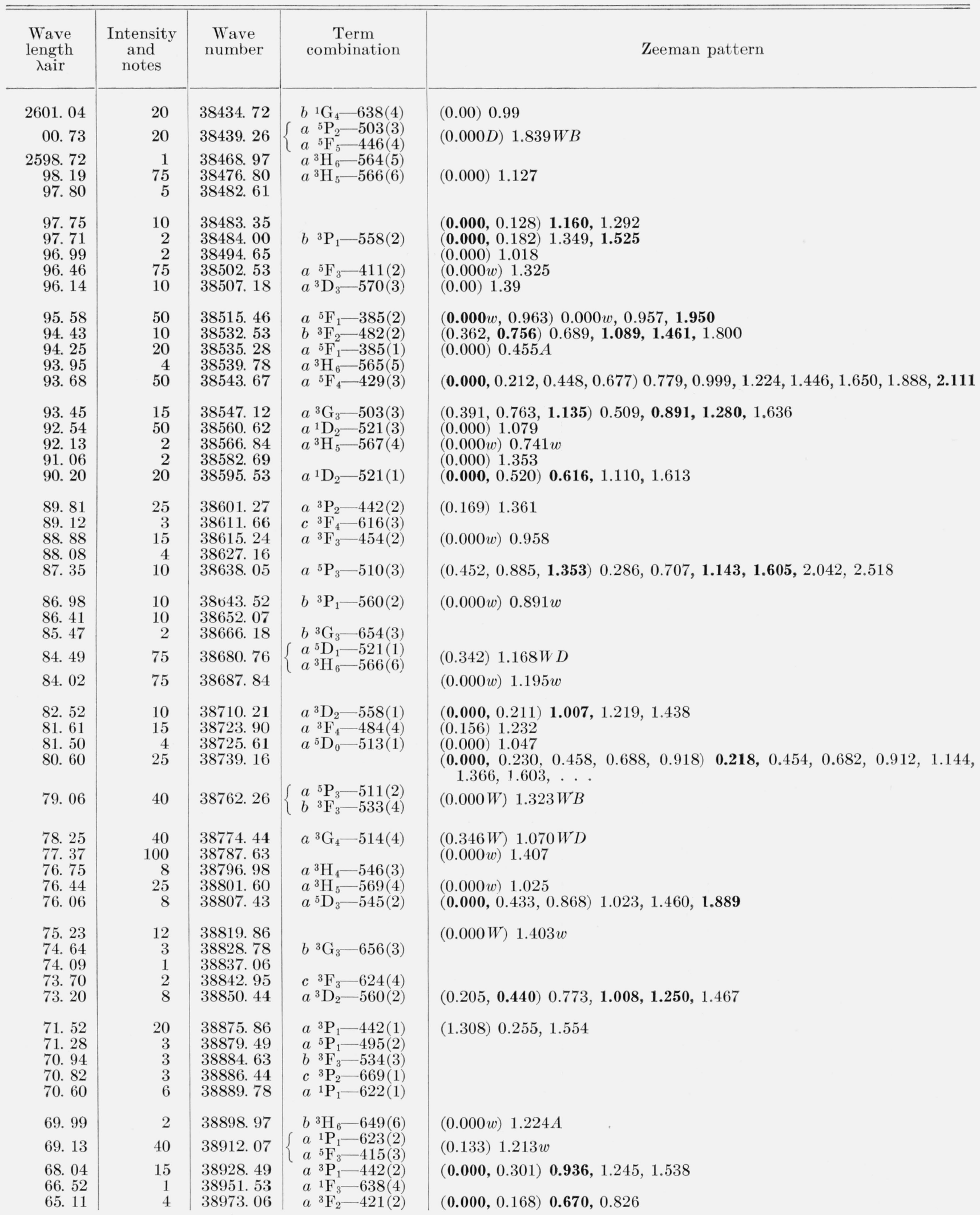


TABLE 1. Wavelengths, term combinations, and Zeeman effects of Ta $\mathrm{II}$-Continued

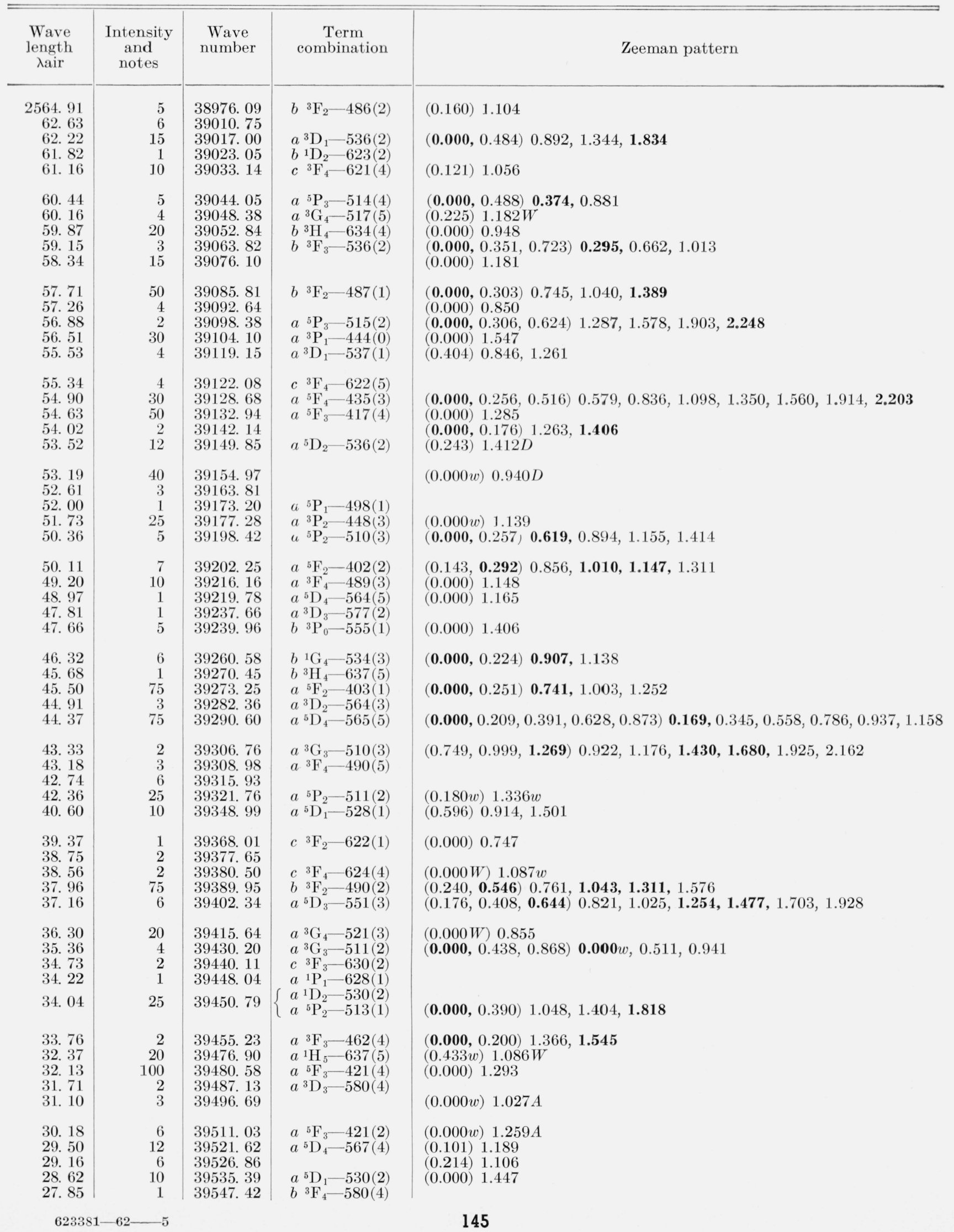


TABLE 1. Wavelengths, term combinations, and Zeeman effects of Ta II-Continued

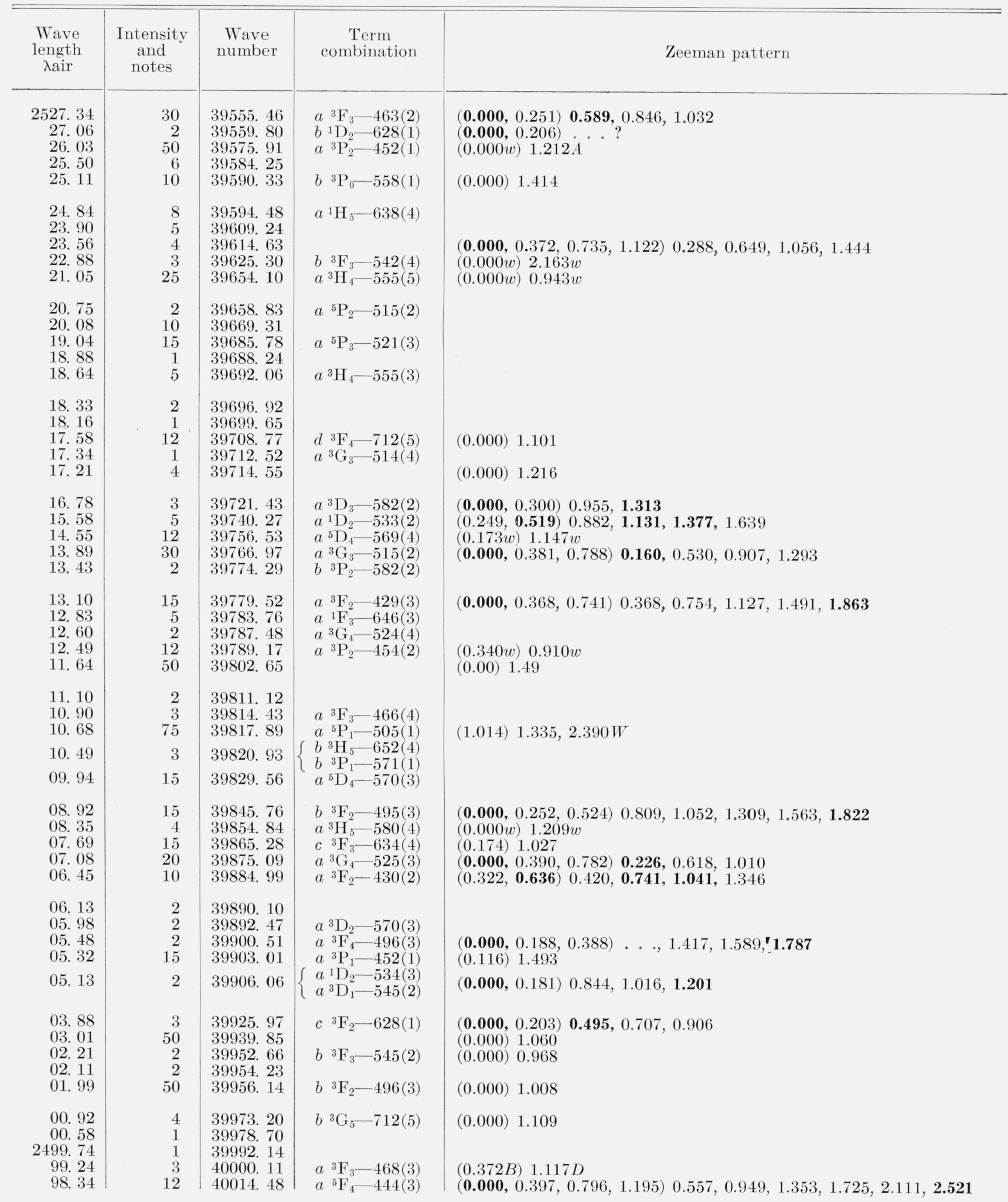


TABLE 1. Wavelengths, term combinations, and Zeeman effects of Ta II-Continued

\begin{tabular}{|c|c|c|c|c|}
\hline $\begin{array}{l}\text { Wave } \\
\text { length } \\
\text { 入air }\end{array}$ & $\begin{array}{l}\text { Intensity } \\
\text { and } \\
\text { notes }\end{array}$ & $\begin{array}{l}\text { Wave } \\
\text { number }\end{array}$ & $\begin{array}{l}\text { Term } \\
\text { combination }\end{array}$ & Zeeman pattern \\
\hline $\begin{array}{r}\text { 2498. } 04 \\
97.77 \\
97.51 \\
97.15 \\
96.24\end{array}$ & $\begin{array}{r}5 \\
10 \\
2 \\
2 \\
10\end{array}$ & $\begin{array}{l}\text { 40019. } 32 \\
40023.65 \\
40027.81 \\
40033.58 \\
40048.18\end{array}$ & $\begin{array}{l}a{ }^{3} \mathrm{~F}_{3}-468(2) \\
a{ }^{5} \mathrm{~F}_{1}-400(0) \\
a^{3} \mathrm{D}_{2}-571(1) \\
b^{3} \mathrm{G}_{4}-682(5)\end{array}$ & $\begin{array}{l}(0.000) 1.018 w \\
(0.118) 0.000 w, 1.018 \\
(0.090, \mathbf{0 . 2 7 6}) . . . ? \\
(0.000) 1.173\end{array}$ \\
\hline $\begin{array}{l}95.03 \\
94.33 \\
94.17 \\
93.97 \\
93.56\end{array}$ & $\begin{array}{l}3 \\
8 \\
6 \\
7 \\
2\end{array}$ & $\begin{array}{l}\text { 40067. } 60 \\
40078.84 \\
\text { 40081. } 41 \\
\text { 40084. } 62 \\
\text { 40091. } 22\end{array}$ & 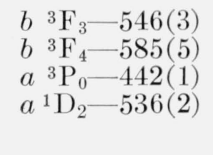 & $\begin{array}{l}(0.178 B) 0.965 w \\
(0.000 D) 0.761 A \\
(0.000) 0.262 \\
(0.224, \mathbf{0 . 4 7 7}) 0.856, \mathbf{1 . 0 9 1}, \mathbf{1 . 3 2 9}, 1.562\end{array}$ \\
\hline $\begin{array}{l}\text { 93. } 14 \\
92.51\end{array}$ & $\begin{array}{l}2 \\
4\end{array}$ & $\begin{array}{l}\text { 40097. } 97 \\
\text { 40108. } 10\end{array}$ & $a^{5} \mathrm{~F}_{5}-462(5)$ & $\begin{array}{l}(0.00) 1.20 \\
(0.427,0.661, \mathbf{0 . 8 9 4}) \\
\quad 1.810 .471,0.617,0.850,1.026, \mathbf{1 . 2 0 7}, \mathbf{1 . 3 8 3}, 1.590,\end{array}$ \\
\hline $\begin{array}{l}\text { 92. } 16 \\
\text { 92. } 01 \\
90.74\end{array}$ & $\begin{array}{r}12 \\
12 \\
2\end{array}$ & $\begin{array}{l}\text { 40113. } 74 \\
\text { 40116. } 15 \\
\text { 40136. } 60\end{array}$ & $\begin{array}{l}a{ }^{5} \mathrm{~F}_{2}-411(2) \\
a^{3} \mathrm{P}_{1}-454(2)\end{array}$ & $\begin{array}{l}(0.295 B) 1.093 D \\
(\mathbf{0 . 0 0 0}, 0.397) \mathbf{0 . 7 7 6}, 1.157,1.542\end{array}$ \\
\hline 90.27 & 20 & 40144. 18 & $a^{5} \mathrm{P}_{3}-525(3)$ & $(0.406 w) 1.165 D$ \\
\hline 88. 71 & 20 & 40169. 40 & $\begin{array}{l}a^{5} \mathrm{~F}_{4}-445(5) \\
a^{5} \mathrm{D}_{1}-536(2)\end{array}$ & $(0.000 \mathrm{~W}) 1.049 \mathrm{~A}$ \\
\hline $\begin{array}{l}87.65 \\
87.34 \\
87.26\end{array}$ & $\begin{array}{r}15 \\
8 \\
3\end{array}$ & $\begin{array}{l}\text { 40186. } 49 \\
\text { 40191. } 38 \\
\text { 40192. } 76\end{array}$ & $\begin{array}{l}a^{1} \mathrm{D}_{2}-537(1) \\
a^{3} \mathrm{~F}_{4}-499(4)\end{array}$ & $\begin{array}{l}(0.000 W) 0.997 A \\
(0.000) 1.592\end{array}$ \\
\hline $\begin{array}{l}87.04 \\
86.81 \\
86.18 \\
85.37 \\
84.75\end{array}$ & $\begin{array}{r}8 \\
5 \\
3 \\
4 \\
10\end{array}$ & $\begin{array}{l}\text { 40196. } 34 \\
\text { 40199. } 98 \\
\text { 40210. } 21 \\
\text { 40223. } 38 \\
\text { 40233. } 32\end{array}$ & $\begin{array}{l}b{ }^{3} \mathrm{~F}_{2}-498(1) \\
c{ }^{3} \mathrm{~F}_{3}-638(4) \\
a \\
{ }^{5} \mathrm{~F}_{4}-446(4) \\
a^{5} \mathrm{D}_{0}-528(1) \\
a^{5} \mathrm{~F}_{1}-402(2)\end{array}$ & $\begin{array}{l}(0.000) 1.049 \\
(0.000) 1.151 \\
(0.268 B) 1.282 D \\
(\mathbf{0 . 0 0 0 ,} 1.170) 1.097,2.301\end{array}$ \\
\hline $\begin{array}{l}\text { 83. } 48 \\
82.60 \\
82.39 \\
81.87 \\
81.15\end{array}$ & $\begin{array}{r}3 \\
30 \\
3 \\
30 \\
3\end{array}$ & $\begin{array}{l}\text { 40253. } 89 \\
\text { 40268. } 26 \\
\text { 40271. } 60 \\
\text { 40280. } 04 \\
\text { 40291. } 76\end{array}$ & $\begin{array}{l}b^{3} \mathrm{D}_{3}-708(2) \\
a{ }^{5} \mathrm{P}_{2}-521(1) \\
a^{3} \mathrm{H}_{4}-561(4)\end{array}$ & $\begin{array}{l}(\mathbf{0 . 0 0 0 ,} 0.228) 1.580,1.775, \mathbf{1 . 9 9 2} \\
(0.000 W) 1.621 B \\
(\mathbf{0 . 0 0 0 ,} 0.231) \mathbf{1 . 1 4 2 ,} 1.373,1.604 \\
(\mathbf{0 . 0 0 0 ,} 0.476,0.945) \\
0.987, \mathbf{1 . 4 8 7}\end{array}$ \\
\hline $\begin{array}{l}80.61 \\
80.37 \\
79.59 \\
79.22 \\
78.45\end{array}$ & $\begin{array}{r}2 \\
3 \\
12 \\
10 \\
1\end{array}$ & $\begin{array}{l}\text { 40300. } 46 \\
\text { 40304. } 43 \\
\text { 40317. } 07 \\
\text { 40323. } 17 \\
\text { 40335. } 60\end{array}$ & 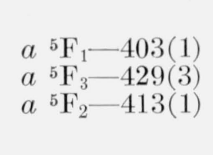 & $\begin{array}{l}(1.206) 0.000 D, 1.281 \\
(0.378 B) 1.202 D \\
(\mathbf{0 . 0 0 0}, 0.877) \mathbf{0 . 1 1 8}, 0.986,1.883\end{array}$ \\
\hline $\begin{array}{l}\text { 78. } 33 \\
77.18 \\
76.69 \\
76.11 \\
75.32\end{array}$ & $\begin{array}{r}3 \\
5 \\
40 \\
3 \\
50\end{array}$ & $\begin{array}{l}\text { 40337. } 50 \\
\text { 40356. } 28 \\
\text { 40364. } 26 \\
\text { 40373. } 76 \\
\text { 40386. } 55\end{array}$ & $\begin{array}{l}a^{3} \mathrm{~F}_{3}-471(3) \\
a^{3} \mathrm{~F}_{2}-435(3) \\
a^{3} \mathrm{~F}_{2}-435(1) \\
a^{3} \mathrm{H}_{5}-585(5)\end{array}$ & 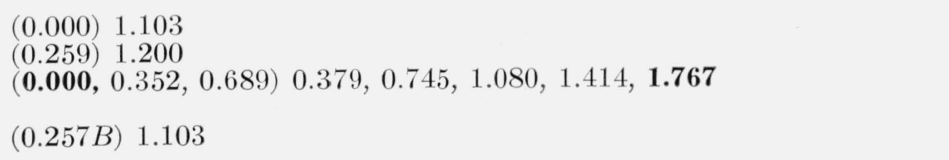 \\
\hline $\begin{array}{l}\text { 74. } 33 \\
73.32 \\
73.12 \\
72.33 \\
71.90\end{array}$ & $\begin{array}{r}5 \\
15 \\
15 \\
6 \\
4\end{array}$ & $\begin{array}{l}\text { 40402. } 74 \\
\text { 40419. } 30 \\
\text { 40422. } 57 \\
\text { 40435. } 47 \\
40442.49\end{array}$ & $\begin{array}{l}c{ }^{3} \mathrm{~F}_{4}-634(4) \\
a{ }^{5} \mathrm{~F}_{4}-448(3) \\
a \\
{ }^{5} \mathrm{~F}_{3}-430(2) \\
a^{1} \mathrm{G}_{4}-546(3)\end{array}$ & $\begin{array}{l}(0.000) 0.872 \\
(0.000 w) 1.510 D \\
(\mathbf{0 . 0 0 0}, 0.186,0.376) 0.946,1.166,1.394, \mathbf{1 . 6 2 2}\end{array}$ \\
\hline $\begin{array}{l}71.48 \\
70.90\end{array}$ & $\begin{array}{r}4 \\
50\end{array}$ & $\begin{array}{l}\text { 40449. } 36 \\
40458.82\end{array}$ & $\begin{array}{l}a{ }^{3} \mathrm{~F}_{3}-472(4) \\
a{ }^{5} \mathrm{~F}_{5}-466(4)\end{array}$ & $\begin{array}{l}(\mathbf{0 . 0 0 0}, 0.166,0.377,0.592,0.798) \\
\quad 2.031, \mathbf{2 . 2 1 4}\end{array}$ \\
\hline $\begin{array}{l}\text { 70. } 69 \\
69.37 \\
68.42\end{array}$ & $\begin{array}{r}3 \\
25 \\
40\end{array}$ & $\begin{array}{l}\text { 40462. } 29 \\
\text { 40483. } 96 \\
\text { 40499. } 48\end{array}$ & $\begin{array}{l}a{ }^{5} \mathrm{P}_{1}-511(2) \\
a^{3} \mathrm{H}_{4}-563(3)\end{array}$ & $\begin{array}{l}(\mathbf{0 . 0 0 0}, 1.021) \mathbf{0 . 3 3 7}, 1.353,2.376 \\
(\mathbf{0 . 0 0 0}, 0.202,0.436,0.633) \mathbf{0 . 3 6 4}, 0.546,0.766 .0 .995,1.210,1.417\end{array}$ \\
\hline $\begin{array}{l}\text { 68. } 10 \\
\text { 67. } 38 \\
\text { 66. } 99 \\
\text { 66. } 66 \\
\text { 65. } 52\end{array}$ & $\begin{array}{r}2 \\
15 \\
15 \\
3 \\
10\end{array}$ & $\begin{array}{l}\text { 40504. } 74 \\
\text { 40516. } 60 \\
\text { 40522. } 98 \\
\text { 40528. } 46 \\
\text { 40547. } 20\end{array}$ & $\begin{array}{l}a{ }^{3} \mathrm{P}_{2}-461(1) \\
a \\
{ }^{5} \mathrm{~F}_{2}-415(3) \\
b \\
{ }^{3} \mathrm{~F}_{3}-551(3)\end{array}$ & 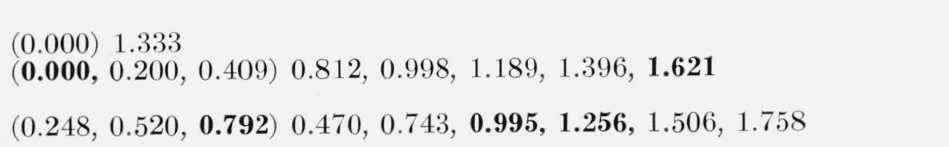 \\
\hline
\end{tabular}


TABLE 1. Wavelengths, term combinations, and Zeeman effects of Ta II-Continued

\begin{tabular}{|c|c|c|c|c|}
\hline $\begin{array}{l}\text { Wave } \\
\text { length } \\
\quad \lambda \text { air }\end{array}$ & $\begin{array}{l}\text { Intensity } \\
\text { and } \\
\text { notes }\end{array}$ & $\begin{array}{l}\text { Wave } \\
\text { number }\end{array}$ & $\begin{array}{l}\text { Term } \\
\text { combination }\end{array}$ & Zeeman pattern \\
\hline $\begin{array}{r}2464.92 \\
64.24 \\
63.83 \\
62.88 \\
62.34\end{array}$ & $\begin{array}{r}3 \\
10 \\
50 \\
12 \\
2\end{array}$ & $\begin{array}{l}40557.00 \\
40568.16 \\
40574.93 \\
40590.60 \\
40599.58\end{array}$ & $\begin{array}{l}a{ }^{3} \mathrm{~F}_{4}-503(3) \\
a{ }^{5} \mathrm{P}_{3}-530(2) \\
a^{3} \mathrm{H}_{6}-585(5) \\
b^{3} \mathrm{H}_{6}-666(5) \\
a^{3} \mathrm{H}_{4}-564(5)\end{array}$ & $\begin{array}{l}(\mathbf{0 . 0 0 0 ,} 0.313,0.606) 0.716,1.036, \mathbf{1 . 3 3 3} \\
(0.000 w) 1.122 A \\
(0.000 w) 1.963 B \\
(0.000 w) 1.180 \\
(0.000 w) 1.119 A\end{array}$ \\
\hline $\begin{array}{l}\text { 61. } 58 \\
61.06 \\
60.91 \\
60.00 \\
58.19\end{array}$ & $\begin{array}{r}7 \\
15 \\
7 \\
10 \\
12\end{array}$ & $\begin{array}{l}40612.03 \\
40620.68 \\
40623.05 \\
40638.13 \\
40668.03\end{array}$ & $\begin{array}{l}a{ }^{5} \mathrm{P}_{1}-513(1) \\
c{ }^{3} \mathrm{~F}_{4}-637(5) \\
a^{3} \mathrm{D}_{2}-577(2) \\
a^{3} \mathrm{G}_{4}-533(4)\end{array}$ & $\begin{array}{ll}(0.000) & 2.375 D \\
(0.156, & \mathbf{0 . 3 5 2}) 0.859, \mathbf{1 . 0 3 3}, \mathbf{1 . 2 1 3}, 1.414 \\
(0.278) & 1.067 D \\
(0.000) & 0.851\end{array}$ \\
\hline $\begin{array}{l}\text { 58. } 02 \\
57.27 \\
56.62 \\
55.97 \\
54.70\end{array}$ & $\begin{array}{r}7 \\
8 \\
5 \\
8 \\
15\end{array}$ & $\begin{array}{l}40670.78 \\
40683.32 \\
40694.05 \\
40704.86 \\
40725.80\end{array}$ & $\left\{\begin{array}{l}a^{3} \mathrm{H}_{4}-565(5) \\
a{ }^{1} \mathrm{H}_{5}-649(5) \\
a^{3} \mathrm{~F}_{3}-475(2) \\
b{ }^{3} \mathrm{P}_{1}-580(1) \\
a \\
a^{5} \mathrm{P}_{2}-525(3) \\
a^{3} \mathrm{G}_{3}-524(4)\end{array}\right.$ & $\begin{array}{l}(\mathbf{0 . 0 0 0}, 0.213,0.433) \mathbf{0 . 6 5 3}, 0.872,1.081,1.287,1.468 \\
(0.000) 1.161 \\
(0.000) \\
(\mathbf{0 . 0 0 0}, 0.193,0.416,0.629) \\
0.700,0.904,1.090,1.328,1.537, \mathbf{1 . 7 2 6}\end{array}$ \\
\hline $\begin{array}{l}54.50 \\
53.98 \\
52.60 \\
51.64 \\
51.51\end{array}$ & $\begin{array}{r}2 \\
15 \\
10 \\
2 \\
1\end{array}$ & $\begin{array}{l}40729.18 \\
40737.86 \\
40760.78 \\
40776.69 \\
40778.84\end{array}$ & $\begin{array}{l}a{ }^{3} \mathrm{P}_{2}-463(2) \\
c{ }^{3} \mathrm{~F}_{4}-638(4) \\
a^{3} \mathrm{G}_{4}-534(3)\end{array}$ & $\begin{array}{l}(0.000) 1.274 \\
(0.302 B) 1.077 D \\
(0.000 W) 0.817 w \\
(0.000) 1.166\end{array}$ \\
\hline $\begin{array}{l}\text { 50. } 99 \\
50.23 \\
49.84 \\
49.65 \\
49.45\end{array}$ & $\begin{array}{r}4 \\
7 \\
8 \\
2 \\
25\end{array}$ & $\begin{array}{l}40787.42 \\
40800.19 \\
40806.67 \\
40809.80 \\
40813.18\end{array}$ & $\begin{array}{l}b{ }^{3} \mathrm{~F}_{3}-553(4) \\
a{ }^{5} \mathrm{D}_{4}-580(4) \\
a^{3} \mathrm{G}_{3}-525(3)\end{array}$ & $(0.483,0.974, \mathbf{1 . 4 9 2}) 0.000,0.411, \mathbf{0 . 8 9 4}, \mathbf{1 . 4 0 3}, 1.884,2.365$ \\
\hline $\begin{array}{l}\text { 48. } 99 \\
\text { 47. } 79 \\
\text { 47. } 61 \\
\text { 47. } 16 \\
45.56\end{array}$ & $\begin{array}{r}10 \\
4 \\
10 \\
25 \\
20\end{array}$ & $\begin{array}{l}\text { 40820. } 83 \\
\text { 40840. } 74 \\
40843.88 \\
40851.24 \\
\text { 40878. } 04\end{array}$ & $\begin{array}{ll}a & { }^{5} \mathrm{P}_{1}-515(2) \\
a & { }^{3} \mathrm{P}_{1}-461(1) \\
b & { }^{3} \mathrm{P}_{2}-593(2)\end{array}$ & $\begin{array}{l}(0.000) 1.300 \\
(0.199) 1.359,1.567 \\
(0.286, \mathbf{0 . 6 2 6}) \\
(0.000) 1.226\end{array}$ \\
\hline $\begin{array}{l}\text { 45. } 18 \\
\text { 44. } 69 \\
\text { 44. } 24 \\
\text { 44. } 13 \\
\text { 43. } 78\end{array}$ & $\begin{array}{r}1 \\
50 \\
4 \\
25 \\
8\end{array}$ & $\begin{array}{l}\text { 40884. } 40 \\
\text { 40892. } 59 \\
\text { 40900. } 12 \\
\text { 40901. } 93 \\
\text { 40907. } 77\end{array}$ & $\left\{\begin{array}{l}b{ }^{3} \mathrm{P}_{1}-582(2) \\
a^{3} \mathrm{H}_{4}-567(4) \\
a \\
{ }^{5} \mathrm{~F}_{3}-435(3) \\
a^{5} \mathrm{P}_{3}-533(4)\end{array}\right.$ & $\begin{array}{l}(0.000 w) 0.000 w \\
(0.357 D) 1.109 D \\
(0.000) 1.723\end{array}$ \\
\hline $\begin{array}{l}\text { 43. } 00 \\
\text { 42. } 81 \\
\text { 42. } 60 \\
\text { 41. } 85 \\
\text { 41. } 34\end{array}$ & $\begin{array}{r}7 \\
7 \\
3 \\
15 \\
12\end{array}$ & $\begin{array}{l}40920.88 \\
40924.03 \\
40927.53 \\
40940.08 \\
40948.73\end{array}$ & $\begin{array}{l}a{ }^{3} \mathrm{D}_{1}-555(2) \\
a{ }^{5} \mathrm{P}_{2}-528(1)\end{array}$ & $\begin{array}{l}(\mathbf{0 . 0 0 0}, 0.225) 0.844,1.078, \mathbf{1 . 3 0 0} \\
(0.000 w) 1.069 A \\
(\mathbf{0 . 0 0 0 ,} 0.514) 0.914,1.407, \mathbf{1 . 8 6 9}\end{array}$ \\
\hline $\begin{array}{l}40.53 \\
40.04 \\
39.81 \\
39.14 \\
38.93\end{array}$ & $\begin{array}{r}12 \\
12 \\
10 \\
1 \\
3\end{array}$ & $\begin{array}{l}40962.24 \\
40970.60 \\
40974.43 \\
40985.55 \\
40989.09\end{array}$ & $\begin{array}{ll}b & { }^{3} \mathrm{~F}_{3}-555(3) \\
b & { }^{3} \mathrm{~F}_{3}-555(2)\end{array}$ & $\begin{array}{l}(0.446,0.868, \mathbf{1 . 3 4 9}) 0.561, \mathbf{0 . 9 9 2 , 1 . 4 1 8 , 1 . 8 4 2} \\
(0.000) 0.942 \\
(0.000 w) 1.023 A\end{array}$ \\
\hline $\begin{array}{l}38.64 \\
38.21 \\
38.04 \\
37.75 \\
36.89\end{array}$ & $\begin{array}{r}20 \\
25 \\
2 \\
15 \\
2\end{array}$ & $\begin{array}{l}40994.03 \\
41001.20 \\
41004.12 \\
41009.02 \\
41023.40\end{array}$ & $\begin{array}{l}a{ }^{3} \mathrm{~F}_{3}-478(3) \\
a^{1} \mathrm{H}_{5}-652(4)\end{array}$ & $\begin{array}{l}(0.334 B) 1.141 D \\
(0.000 w) 1.038 W A\end{array}$ \\
\hline $\begin{array}{l}36.73 \\
36.51 \\
36.31 \\
35.59 \\
34.93\end{array}$ & $\begin{array}{r}2 \\
25 \\
2 \\
4 \\
5\end{array}$ & $\begin{array}{l}\text { 41026. } 20 \\
41029.94 \\
41033.24 \\
41045.30 \\
41056.49\end{array}$ & $\begin{array}{r}a^{3} \mathrm{~F}_{2}-442(1) \\
a^{5} \mathrm{P}_{3}-534(3) \\
\left\{\begin{array}{l}c^{3} \mathrm{~F}_{3}-646(3) \\
a^{5} \mathrm{D}_{2}-555(1)\end{array}\right. \\
\left\{\begin{array}{l}a^{3} \mathrm{P}_{1}-463(2) \\
a^{5} \mathrm{D}_{2}-555(2)\end{array}\right.\end{array}$ & 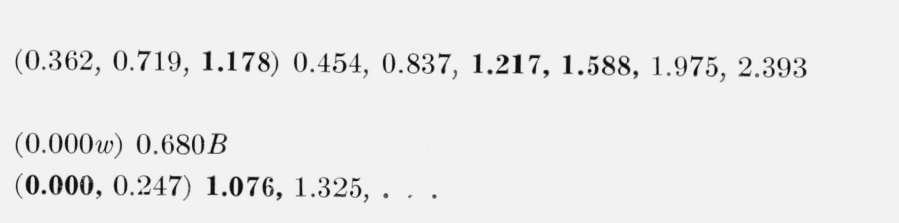 \\
\hline
\end{tabular}


TABLE 1. Wavelengths, term combinations, and Zeeman effects of Ta II-Continued

\begin{tabular}{|c|c|c|c|c|}
\hline $\begin{array}{c}\text { Wave } \\
\text { length } \\
\lambda \text { air }\end{array}$ & $\begin{array}{c}\text { Intensity } \\
\text { and } \\
\text { notes }\end{array}$ & $\begin{array}{l}\text { Wave } \\
\text { number }\end{array}$ & $\begin{array}{l}\text { Term } \\
\text { combination }\end{array}$ & Zeeman pattern \\
\hline 2434. 82 & 5 & 41058. 34 & $a^{5} \mathrm{D}_{1}-545(2)$ & $(\mathbf{0 . 0 0 0}, 0.487) \mathbf{0 . 5 5 2}, 1.029,1.514$ \\
\hline 33.59 & 20 & 41079. 13 & $a^{3} \mathrm{~F}_{2}-442(2)$ & $(0.504, \mathbf{1 . 0 1 9}) 0.245, \mathbf{0 . 7 4 4}, \mathbf{1 . 2 4 8}, 1.754$ \\
\hline 33. 03 & 8 & 41088. 57 & $a^{1} \mathrm{D}_{2}-546(3)$ & (0 000, $0.180,0.356) \quad \mathbf{0 . 5 6 9}, 0.772,0.962,1.179,1.368$ \\
\hline 32. 71 & 75 & 41093. 92 & $a^{5} \mathrm{~F}_{5}-472(4)$ & 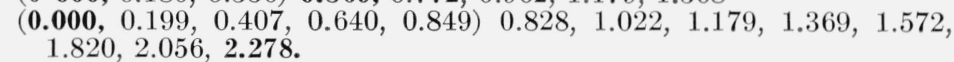 \\
\hline 31. 97 & 12 & 41106. 49 & $a^{3} \mathrm{D}_{2}-582(2)$ & $(0.433, \mathbf{0 . 8 7 7}) 0.740, \mathbf{1 . 2 0 8}, 1.643$ \\
\hline 31. 81 & 6 & 41109. 16 & $a{ }^{3} \mathrm{P}_{0}-452(1)$ & $(0.000) 1.474$ \\
\hline 31. 06 & 15 & 41121.93 & $a^{5} \mathrm{~F}_{2}-421(2)$ & $(0.201, \mathbf{0 . 4 4 2}) 0.785, \mathbf{0 . 9 8 9}, \mathbf{1 . 2 1 3}, 1.435$ \\
\hline 30.51 & 15 & 41131. 20 & $b^{3} \mathrm{H}_{5}-665(4)$ & \\
\hline 30. 22 & 30 & 41136. 04 & $a^{5} \mathrm{P}_{2}-530(2)$ & $(0.000 w) 1.133$ \\
\hline 29. 68 & 25 & 41145. 16 & $a^{5} \mathrm{~F}_{1}-411(2)$ & $(\mathbf{0 . 0 0 0} w, 1.128) 0.000 w, 1.150,2.268$ \\
\hline 28. 50 & 30 & 41165. 19 & & \\
\hline 28. 00 & 20 & 41173. 60 & $a{ }^{3} \mathrm{P}_{2}-468(3)$ & $(0.000 w) 0.973 D$ \\
\hline 26. 95 & 4 & 41191. 46 & & \\
\hline 26. 86 & 7 & 41192. 97 & $a^{3} \mathrm{P}_{2}-468(2)$ & $(0.393, \mathbf{0 . 4 8 8}) 0.860, \mathbf{1 . 1 0 1}, \mathbf{1 . 3 3 4}, 1.596$ \\
\hline 26.53 & 4 & 41198. 60 & $b^{3} \mathrm{~F}_{4}-596(5)$ & $(\mathbf{0 . 0 0 0}, 0.265,0.537,0.820,1.106) \mathbf{0 . 9 5 8}, 1.222, \ldots$ \\
\hline 25. 91 & 30 & 41209. 15 & $a^{5} \mathrm{P}_{3}-536(2)$ & $(\mathbf{0 . 0 0 0}, 0.258,0.577) \ldots, 1.093,1.353,1.532,1.9,2.2$ \\
\hline 25. 47 & 10 & 41216. 61 & & $(0.000 w) 0.698 A$ \\
\hline 25. 20 & 12 & 41221. 20 & & \\
\hline 25. 13 & 3 & 41222. 42 & & $(0.000 w) 1.568$ \\
\hline 24. 97 & 3 & 41225. 09 & $b^{1} \mathrm{G}_{4}-666(5)$ & $(0.000) \quad 0.966$ \\
\hline 24. 58 & 2 & 41231. 80 & $a^{3} \mathrm{D}_{1}-558(2)$ & $(\mathbf{0 . 0 0 0}, 0.486) 1.741$ \\
\hline 24. 05 & 3 & 41240. 70 & & \\
\hline 23. 89 & 8 & 41243. 51 & $a^{3} \mathrm{G}_{3}-530(2)$ & $(\mathbf{0 . 0 0 0}, 0.552,1.102) \mathbf{0 . 3 3 5}, 0.899,1.470,1.980$ \\
\hline 23. 49 & 20 & 41250. 32 & $a^{3} \mathrm{~F}_{2}-444(3)$ & $(\mathbf{0 . 0 0 0}, 0.196,0.398) 0.564,0.753,0.946,1.128, \mathbf{1 . 3 4 0}$ \\
\hline 21. 85 & 50 & 41278. 25 & $b^{3} \mathrm{~F}_{3}-558(2)$ & $(\mathbf{0 . 0 0 0}, 0.317,0.670) \mathbf{0 . 3 2 4}, 0.674,0.987,1.306,1.626$ \\
\hline 20. 83 & 2 & 41295. 67 & & $(0.483) 1.212 C$ \\
\hline 20.35 & 1 & 41303. 78 & & \\
\hline 18. 95 & $\begin{array}{r}6 \\
20\end{array}$ & 41327. 76 & $a^{3} \mathrm{~F}_{4}-510(3)$ & $(\mathbf{0 . 0 0 0 D}) 1.347 w$ \\
\hline 18. 77 & 20 & 41330. 77 & $\int a^{5} \mathrm{D}_{3}-570(3)$ & $(0.000) \quad 0.947$ \\
\hline 18.54 & 2 & 41334. 74 & $\left\{\begin{array}{l}a \\
a^{3} \mathrm{~F}_{3}-481(2)\end{array}\right.$ & \\
\hline 18. 31 & 3 & 41338. 63 & & \\
\hline 18. 10 & 10 & 41342. 31 & & \\
\hline 17. 85 & 30 & 41346. 53 & & $(0.332 w) 1.138 D$ \\
\hline 17. 38 & 4 & 41354. 54 & & \\
\hline 17. 33 & 5 & 41355. 39 & $a{ }^{5} \mathrm{~F}_{1}-413(1)$ & \\
\hline 17. 15 & 7 & 41358. 44 & $b^{1} \mathrm{D}_{2}-646(3)$ & $(0.000) 1.023$ \\
\hline 16. 89 & 25 & 41362. 87 & $a^{5} \mathrm{~F}_{3}-440(4)$ & $(0.000 D) 0.738 A$ \\
\hline 16. 73 & 1 & 41365. 66 & & \\
\hline 15. 83 & 1 & 41381. 13 & & \\
\hline 15. 70 & 1 & 41383. 24 & $b^{3} \mathrm{~F}_{2}-510(3)$ & \\
\hline 15. 24 & 15 & 41391. 11 & $a^{3} \mathrm{D}_{1}-560(2)$ & $(0.000 w) 1.044 A$ \\
\hline 15. 22 & 12 & 41391. 48 & $a^{3} \mathrm{~F}_{3}-482(2)$ & $(\mathbf{0 . 0 0 0}, 0.361,741) \mathbf{0 . 3 6 4}, 0.715$ \\
\hline 13. 78 & 4 & 41416. 16 & & \\
\hline $\begin{array}{l}12.54 \\
12.27\end{array}$ & $\begin{array}{r}12 \\
8\end{array}$ & $\begin{array}{l}41437.58 \\
41442.13\end{array}$ & $\begin{array}{ll}b & { }^{3} \mathrm{~F}_{3}-560(2) \\
a & { }^{5} \mathrm{P}_{1}-521(1)\end{array}$ & $\begin{array}{l}(0.000) 1.006 \\
(0.764 D) 1.618,2.326\end{array}$ \\
\hline & 3 & 41470.63 & ${ }^{3} \mathrm{G}_{3}-682(3)$ & $(0.539) 0.141 \quad 0.770$ \\
\hline 09.80 & 5 & 41484. 69 & & $(\mathbf{0 . 0 0 0}, 0.283,0.610,0.895) \ldots, 1.475,1.746,2.041$ \\
\hline 08.83 & 8 & 41501. 24 & $a^{3} \mathrm{G}_{4}-542(4)$ & \\
\hline 08. 50 & 5 & 41506. 96 & $b{ }^{3} \mathrm{~F}_{2}-511(2)$ & $(0.316, \mathbf{0 . 6 2 5}) \quad 0.722, \mathbf{1 . 0 4 5}, \mathbf{1 . 3 5 2}, 1.670$ \\
\hline 08. 25 & 20 & 41511. 27 & $a^{3} \mathrm{P}_{2}-471(3)$ & $(\mathbf{0 . 0 0 0}, 0.260,0.529) \mathbf{0 . 5 7 8}, 0.849,1.092$ \\
\hline 07. 75 & 10 & 41519. 89 & $a^{3} \mathrm{P}_{1}-468(2)$ & $(\mathbf{0 . 0 0 0}, 0.458) \mathbf{0 . 6 5 1}, 1.102,1.562$ \\
\hline 07.54 & 1 & 41523. 58 & $a^{5} \mathrm{D}_{2}-560(2)$ & \\
\hline 06.87 & $\begin{array}{l}2 \\
2\end{array}$ & $\begin{array}{l}41535.07 \\
41554.26\end{array}$ & & \\
\hline $\begin{array}{l}05.76 \\
05.40\end{array}$ & $\begin{array}{l}2 \\
3\end{array}$ & $\begin{array}{l}41554.26 \\
41560.52\end{array}$ & & $(0.232) 0.672,0.948$ \\
\hline 04.80 & 10 & 41570. 82 & $c^{3} \mathrm{~F}_{4}-646(3)$ & (0.000) 0.961 \\
\hline 04. 48 & 10 & 41576. 38 & $a^{3} \mathrm{G}_{3}-533(4)$ & $(\mathbf{0 . 0 0 0}, 0.189,0.381,0.561) \quad 0.897,1.115,1.283,1.477, \mathbf{1 . 6 4 3}$ \\
\hline 03. 68 & 25 & 41590. 22 & $a^{5} \mathrm{P}_{2}-534(3)$ & $(\mathbf{0 . 0 0 0}, 0.216,0.455) \quad 0.789,0.988,1.220,1.442$ \\
\hline 03.54 & 3 & 41592. 71 & & $(0.000 \mathrm{w}) 1.078$ \\
\hline 03.13 & 10 & 41599. 70 & & $(0.000 D) 1.417$ \\
\hline
\end{tabular}


TABLE 1. Wavelengths, term combinations, and Zeeman effects of Ta II-Continued

\begin{tabular}{|c|c|c|c|c|}
\hline $\begin{array}{c}\text { Wave } \\
\text { length } \\
\lambda \text { air }\end{array}$ & $\begin{array}{c}\text { Intensity } \\
\text { and } \\
\text { notes }\end{array}$ & $\begin{array}{l}\text { Wave } \\
\text { number }\end{array}$ & $\begin{array}{l}\text { Term } \\
\text { combination }\end{array}$ & Zeeman pattern \\
\hline 2402. 13 & 30 & 41617. 06 & $a^{5} \mathrm{~F}_{3}-442(2)$ & $(0.000) 1.240$ \\
\hline 01.04 & 6 & 41635.88 & $b^{3} \mathrm{~F}_{2}-513(1)$ & $(0.000) 1.049$ \\
\hline 00.92 & 6 & 41638. 03 & & \\
\hline 00.62 & 100 & 41643. 16 & $a{ }^{5} \mathrm{~F}_{5}-478(6)$ & $(0.000 D) 1.175 D$ \\
\hline 2399. 94 & 15 & 41655. 06 & $a^{3} \mathrm{~F}_{2}-448(3)$ & $(\mathbf{0 . 0 0 0}, 0.516,1.029) 0.247,0.744,1.254,1.744,2.248$ \\
\hline 98. 74 & 4 & 41675.86 & $a^{1} \mathrm{~F}_{3}-665(4)$ & $(0.000 w) 1.441$ \\
\hline 98. 34 & 6 & 41682.85 & & $(0.000 w) 1.211 w$ \\
\hline 98. 10 & 10 & 41686. 97 & $a^{3} \mathrm{D}_{3}-602(3)$ & $(0.390 w) 1.275 D$ \\
\hline $\begin{array}{l}97.76 \\
97.52\end{array}$ & $\begin{array}{l}5 \\
4\end{array}$ & $\begin{array}{l}\text { 41692. } 81 \\
\text { 41697. } 07\end{array}$ & & $(0.286,0.609, \mathbf{0 . 9 2 8}) 0.281,0.614, \mathbf{0 . 9 2 8}, \mathbf{1 . 2 2 4}, 1.552,1.957$ \\
\hline & & (2) & & (1. \\
\hline 97. 44 & 3 & 41698. 46 & $a^{3} \mathrm{G}_{3}-534(3)$ & \\
\hline $\begin{array}{l}96.74 \\
95.94\end{array}$ & $\begin{array}{l}1 \\
3\end{array}$ & 41710.59 & & $(0.355) 1.177 \quad 00$ \\
\hline $\begin{array}{l}95.94 \\
95.40\end{array}$ & $\begin{array}{l}3 \\
3\end{array}$ & 41724.57 & $\begin{array}{l}c{ }^{3} \mathrm{~F}_{2}-646(3) \\
a^{3} \mathrm{~F}_{4}-514(4)\end{array}$ & \\
\hline $\begin{array}{l}\text { 95. } 40 \\
95.03\end{array}$ & $\begin{array}{r}3 \\
12\end{array}$ & $\begin{array}{l}41733.94 \\
41740.42\end{array}$ & $\begin{array}{l}a{ }^{3} \mathrm{~F}_{4}-514(4) \\
b{ }^{3} \mathrm{P}_{2}-602(3)\end{array}$ & $\begin{array}{l}(0.393) 1.212 \\
(\mathbf{0 . 0 0 0}, 0.255,0.556) \mathbf{0 . 6 1 5}, 0.914,1.178,1.443,1.732\end{array}$ \\
\hline 94. 77 & 1 & 41744. 95 & & \\
\hline 94.65 & 5 & 41747. 01 & $b^{3} \mathrm{~F}_{4}-602(3)$ & $(0.000) 1.320$ \\
\hline 93. 27 & 7 & 41771. 15 & $a^{3} \mathrm{D}_{3}-603(3)$ & $(0.497 D) 0.729,0.941, \mathbf{1 . 1 5 6}, \mathbf{1 . 3 6 7}, \ldots$ \\
\hline 92. 70 & 6 & 41781. 13 & $b{ }^{3} \mathrm{P}_{0}-580(1)$ & $(0.000) 1.143$ \\
\hline 92. 29 & 3 & 41788. 19 & $a{ }^{5} \mathrm{~F}_{3}-444(3)$ & $(0.304,0.612, \mathbf{0 . 9 1 1}) 0.320,0.636, \mathbf{0 . 9 5 2}, \mathbf{1 . 2 4 8 , 1 . 5 4 0 , 1 . 8 5 1}$ \\
\hline 90. 71 & 5 & 41815. 77 & & \\
\hline 90. 43 & 2 & 41820.80 & & $(0.000) 1.521$ \\
\hline 90. 22 & 4 & 41824. 37 & $b{ }^{3} \mathrm{P}_{2}-603(3)$ & $(\mathbf{0 . 0 0 0}, 0.314,0.635) \mathbf{0 . 4 6 7}, 0.814,1.130,1.430$ \\
\hline 89. 60 & 8 & 41835. 29 & $a^{3} \mathrm{~F}_{3}-486(2)$ & $(0.000 w) 1.021$ \\
\hline 89. 11 & 10 & 41843. 79 & $b{ }^{3} \mathrm{~F}_{2}-515(2)$ & $(0.235, \mathbf{0 . 4 8 7}) 0.806, \mathbf{1 . 0 5 2}, \mathbf{1 . 2 9 9}, 1.554$ \\
\hline 88. 39 & 15 & 41856. 45 & $\begin{array}{l}a{ }^{5} \mathrm{D}_{2}-563(3) \\
a^{3} \mathrm{P}_{2}-475(2)\end{array}$ & $(0.000 w) 1.303$ \\
\hline 87.54 & 5 & 41871. 39 & $\begin{array}{l}a{ }^{5} \mathrm{~F}_{4}-462(4) \\
a{ }^{5} \mathrm{P}_{2}-537(1)\end{array}$ & $(0.000) 1.508$ \\
\hline 87. 35 & 2 & 41874. 68 & $c{ }^{3} \mathrm{~F}_{3}-654(3)$ & \\
\hline 87. 09 & 50 & 41879. 28 & $a^{5} \mathrm{~F}_{4}-462(5)$ & $(0.579 D) 0.627 W$ \\
\hline 84.51 & 1 & 41924.59 & & \\
\hline 84. 30 & 12 & 41928. 21 & $a^{5} \mathrm{~F}_{2}-429(3)$ & $(0.000 w) 1.314$ \\
\hline 83. 74 & 15 & 41938. 01 & $a^{3} \mathrm{P}_{2}-475(1)$ & $(0.000 w) 1.161$ \\
\hline 83. 15 & 2 & 41948. 42 & & $(0.000) 0.985$ \\
\hline 81. 55 & 40 & 41976.68 & $b{ }^{3} \mathrm{P}_{1}-593(2)$ & $(0.000) 1.122$ \\
\hline 81. 14 & 50 & 41983. 81 & $a^{5} \mathrm{~F}_{3}-446(4)$ & $(0.000) 1.298$ \\
\hline 79. 80 & 2 & 42007.54 & a. ${ }^{3} \mathrm{~F}_{4}-517(5)$ & \\
\hline 78. 33 & 10 & 42033. 52 & $a^{5} \mathrm{~F}_{2}-430(2)$ & $(0.116) 1.038$ \\
\hline 78. 20 & 2 & 42035. 78 & & \\
\hline 77. 18 & $\begin{array}{l}5 \\
8\end{array}$ & $\begin{array}{l}42049.71 \\
42053.78\end{array}$ & $\begin{array}{l}a{ }^{3} \mathrm{P}_{0}-461(1) \\
a^{3} \mathrm{~F}_{2}-452(1)\end{array}$ & 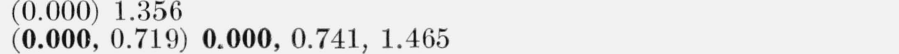 \\
\hline & 3 & & $a^{5} \mathrm{D}_{1}-555(2)$ & $(0.000,0.456) \quad 0.579,1.025$ \\
\hline 74. 69 & 5 & 42097. 91 & ${ }^{5} \mathrm{P}_{3}-545(2)$ & $(\mathbf{0 . 0 0 0}, 0.578,1.145) 0.990,1.458,2.140,2.737$ \\
\hline 74. 20 & 3 & 42106.52 & & \\
\hline 73. 92 & 20 & 42111. 51 & $a{ }^{5} \mathrm{P}_{1}-528(1)$ & $(1.457 B) 0.906,2.394 w$ \\
\hline 72. 81 & 12 & 42131. 23 & $a^{3} \mathrm{~F}_{3}-489(3)$ & $(0.187,0.433, \mathbf{0 . 5 9 5}) 0.683,0.880, \mathbf{1 . 0 7 2}, \mathbf{1 . 2 7 4}, 1.495,1.689$ \\
\hline 71. 58 & 10 & 42153. 18 & $a^{5} \mathrm{~F}_{1}-421(2)$ & $(0.000,1.185) 1.185,2.340$ \\
\hline 71. 06 & 10 & 42162. 44 & & $(\mathbf{0 . 0 0 0}, 0.219,0.453,0.662) \mathbf{0 . 3 2 6}, 0.548,0.767,1.020,1.254,1.487$ \\
\hline 70. 77 & 12 & 42167.51 & $a^{3} \mathrm{P}_{2}-478(3)$ & $(0.000 D) 0.918 A$ \\
\hline $\begin{array}{l}70.52 \\
70.37\end{array}$ & $\begin{array}{l}1 \\
8\end{array}$ & $\begin{array}{l}42171.96 \\
42174.64\end{array}$ & $b{ }^{3} \mathrm{~F}_{3}-567(4)$ & $(0.00) \quad 1.35$ \\
\hline 69.85 & 7 & 42183.95 & $a^{3} \mathrm{P}_{1}-475(2)$ & $(\mathbf{0 . 0 0 0}, 0.266) \mathbf{1 . 0 2 8}, 1.291,1.540$ \\
\hline 69.53 & 4 & 42189.54 & $a^{3} \mathrm{H}_{4}-580(4)$ & $(0.302) 1.058$ \\
\hline 69. 34 & 8 & 42192. 89 & $a{ }^{5} \mathrm{~F}_{3}-448(3)$ & $(0.000) 1.236$ \\
\hline $\begin{array}{l}\text { 68. } 78 \\
68.56\end{array}$ & $\begin{array}{l}2 \\
2\end{array}$ & $\begin{array}{l}42202.99 \\
42206.85\end{array}$ & $b{ }^{3} \mathrm{H}_{6}-682(5)$ & $(0.222) 1.137$ \\
\hline 68. 22 & 5 & 42212.88 & $a{ }^{5} \mathrm{P}_{3}-546(3)$ & $(0.676,1.335, \mathbf{1 . 9 8 4}) 0.295, \mathbf{0 . 9 5 4}, \mathbf{1 . 6 2 0}, 2.262$ \\
\hline 67. 71 & 4 & 42222.00 & & (2) \\
\hline 67. 26 & 15 & 42230. 01 & $a^{5} \mathrm{~F}_{4}-466(4)$ & $(0.437 w) 1.272 D$ \\
\hline 66. 18 & 1 & 42249. 25 & $a^{3} \mathrm{~F}_{3}-490(2)$ & $(\mathbf{0 . 0 0 0}, 0.235,0.462) 0.633, \ldots$ \\
\hline 65. 29 & 2 & 42265. 20 & $a^{3} \mathrm{P}_{1}-475(1)$ & $(0.000) 1.507$ \\
\hline
\end{tabular}


TABLE 1. Wavelengths, term combinations, and Zeeman effects of Ta II-Continued

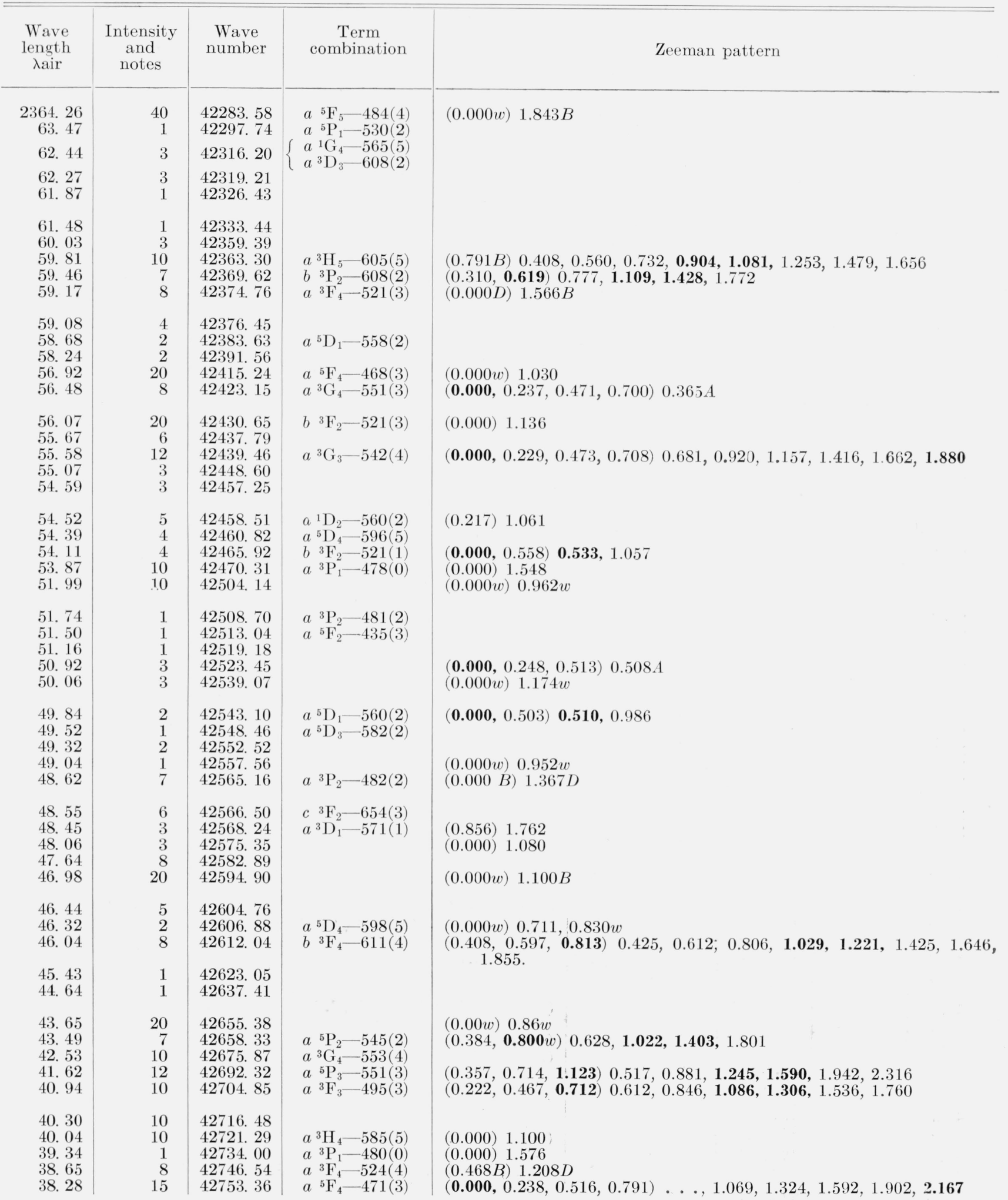


TABLE 1. Wavelengths, term combinations, and Zeeman effects of Ta II-Continued

\begin{tabular}{|c|c|c|c|c|}
\hline $\begin{array}{l}\text { Wave } \\
\text { length } \\
\text { 入air }\end{array}$ & $\begin{array}{l}\text { Intensity } \\
\text { and } \\
\text { notes }\end{array}$ & $\begin{array}{l}\text { Wave } \\
\text { number }\end{array}$ & $\begin{array}{c}\text { Term } \\
\text { combination }\end{array}$ & Zeeman pattern \\
\hline $\begin{array}{r}2337.82 \\
37.56 \\
37.18 \\
36.55\end{array}$ & $\begin{array}{l}2 \\
3 \\
4 \\
8\end{array}$ & $\begin{array}{l}42761.75 \\
42766.61 \\
42773.57 \\
42785.03\end{array}$ & $\begin{array}{l}a{ }^{3} \mathrm{~F}_{3}-495(2) \\
a^{3} \mathrm{G}_{3}-545(2) \\
a^{5} \mathrm{P}_{2}-546(3)\end{array}$ & $(\mathbf{0 . 0 0 0}, 0.478,0.972) \mathbf{0 . 0 0 0}, 0.451,0.932,1.413$ \\
\hline 35. 75 & 15 & 42799. 68 & $\left\{\begin{array}{l}b^{3} \mathrm{H}_{5}-682(5) \\
a^{3} \mathrm{G}_{4}-555(5)\end{array}\right.$ & $(0.000 w) 1.146 w$ \\
\hline $\begin{array}{l}\text { 35. } 49 \\
\text { 34. } 90 \\
\text { 34. } 14 \\
\text { 33. } 87 \\
\text { 33. } 08\end{array}$ & $\begin{array}{r}3 \\
12 \\
20 \\
12 \\
2\end{array}$ & $\begin{array}{l}\text { 42804. } 44 \\
42815.30 \\
42829.27 \\
42834.15 \\
42848.69\end{array}$ & $\begin{array}{ll}a & { }^{5} \mathrm{~F}_{3}-454(2) \\
a & { }^{3} \mathrm{~F}_{3}-496(3) \\
& \\
a & { }^{3} \mathrm{~F}_{4}-525(3) \\
c & { }^{3} \mathrm{P}_{2}-708(2)\end{array}$ & $\begin{array}{l}(0.123) 1.057 \\
(0.000 w) 1.150 \mathrm{~A} \\
(\mathbf{0 . 0 0 0}, 0.198,0.301,0.502) \mathbf{0 . 6 4 3}, 0.716,0.887,1.105,1.349\end{array}$ \\
\hline $\begin{array}{l}\text { 32. } 19 \\
32.00 \\
31.30 \\
\text { 30. } 82 \\
30.70\end{array}$ & $\begin{array}{r}25 \\
35 \\
20 \\
5 \\
2\end{array}$ & $\begin{array}{l}42865.02 \\
42868.53 \\
42881.44 \\
42890.27 \\
42892.41\end{array}$ & $\begin{array}{l}a{ }^{5} \mathrm{~F}_{4}-472(4) \\
a^{5} \mathrm{~F}_{5}-490(5) \\
a^{3} \mathrm{G}_{3}-546(3) \\
b^{3} \mathrm{~F}_{2}-525(3) \\
a^{3} \mathrm{P}_{1}-482(2)\end{array}$ & $\begin{array}{l}(0.60) 0.92 \\
(0.000) 0.886 \\
(0.000 w) 1.370 \\
(0.000) 1.401\end{array}$ \\
\hline $\begin{array}{l}\text { 29. } 88 \\
29.45 \\
29.29 \\
29.20 \\
28.96\end{array}$ & $\begin{array}{l}2 \\
1 \\
1 \\
4 \\
1\end{array}$ & $\begin{array}{l}42907.59 \\
42915.36 \\
42918.37 \\
42919.95 \\
42924.45\end{array}$ & $a^{3} \mathrm{H}_{5}-611(4)$ & $(0.000 w) 1.226 w$ \\
\hline $\begin{array}{l}28.76 \\
28.57 \\
27.64 \\
27.00 \\
26.74\end{array}$ & $\begin{array}{l}3 \\
1 \\
6 \\
4 \\
1\end{array}$ & $\begin{array}{l}\text { 42928. } 21 \\
42931.64 \\
42948.79 \\
42960.60 \\
42965.33\end{array}$ & $a^{5} \mathrm{P}_{1}-536(2)$ & $(0.189) \quad 1.153$ \\
\hline $\begin{array}{l}26.48 \\
25.70 \\
25.15 \\
24.33 \\
23.90\end{array}$ & $\begin{array}{r}6 \\
2 \\
2 \\
2 \\
20\end{array}$ & $\begin{array}{l}42970.29 \\
42984.70 \\
42994.85 \\
43009.96 \\
43017.85\end{array}$ & $\begin{array}{l}a^{3} \mathrm{~F}_{2}-461(1) \\
a^{5} \mathrm{D}_{4}-602(3)\end{array}$ & $\begin{array}{l}(0.000) 1.221 \\
(0.00) 0.99\end{array}$ \\
\hline $\begin{array}{l}\text { 23. } 04 \\
22.21 \\
21.38 \\
21.17 \\
20.93\end{array}$ & $\begin{array}{l}3 \\
2 \\
1 \\
4 \\
1\end{array}$ & $\begin{array}{l}43033.75 \\
43049.21 \\
43064.65 \\
43068.53 \\
43072.89\end{array}$ & $\begin{array}{l}a{ }^{5} \mathrm{P}_{1}-537(1) \\
a \\
{ }^{5} \mathrm{~F}_{1}-430(2) \\
a \\
{ }^{5} \mathrm{~F}_{1}-430(0) \\
a^{3} \mathrm{D}_{2}-602(3)\end{array}$ & $(0.00) 0.00$ \\
\hline $\begin{array}{l}\text { 20. } 58 \\
20.35 \\
19.79 \\
19.51 \\
19.25\end{array}$ & $\begin{array}{l}4 \\
2 \\
2 \\
3 \\
2\end{array}$ & $\begin{array}{l}\text { 43079. } 44 \\
43083.75 \\
\text { 43094. } 08 \\
\text { 43099. } 31 \\
\text { 43104. } 22\end{array}$ & $\begin{array}{l}a^{5} \mathrm{D}_{4}-603(3) \\
b^{3} \mathrm{P}_{1}-604(1)\end{array}$ & \\
\hline $\begin{array}{l}\text { 19. } 09 \\
\text { 18. } 64 \\
18.48 \\
17.66 \\
17.18\end{array}$ & $\begin{array}{l}8 \\
4 \\
2 \\
2 \\
1\end{array}$ & $\begin{array}{l}43107.19 \\
43115.45 \\
43118.46 \\
43133.77 \\
43142.59\end{array}$ & $\begin{array}{ll}a & { }^{5} \mathrm{P}_{3}-555(3) \\
a & { }^{5} \mathrm{P}_{3}-555(2) \\
a^{3} \mathrm{P}_{2} & 487(1) \\
b & { }^{3} \mathrm{~F}_{2}-528(1)\end{array}$ & $\begin{array}{l}(0.440 D) 1.526 D \\
(\mathbf{0 . 0 0 0}, 0.573,1.148) 1.568,2.062,2.647 \\
(0.000 w) 1.144\end{array}$ \\
\hline $\begin{array}{l}\text { 17. } 03 \\
\text { 16. } 70 \\
\text { 16. } 42 \\
\text { 16. } 04 \\
15.65\end{array}$ & $\begin{array}{l}1 \\
3 \\
4 \\
5 \\
3\end{array}$ & $\begin{array}{l}43145.37 \\
43151.62 \\
43156.76 \\
43163.81 \\
43171.15\end{array}$ & $\begin{array}{l}a^{3} \mathrm{D}_{2}-603(3) \\
a^{3} \mathrm{D}_{1}-577(2) \\
a^{3} \mathrm{H}_{5}-613(5)\end{array}$ & $\begin{array}{l}(\mathbf{0 . 0 0 0}, 0.393) 0.494,0.884, \mathbf{1 . 2 8 2} \\
(0.000 w) 0.995 \\
(0.000 w) 0.587 A\end{array}$ \\
\hline $\begin{array}{l}\text { 15. } 45 \\
\text { 14. } 29 \\
\text { 14. } 08 \\
12.93 \\
12.62\end{array}$ & $\begin{array}{r}10 \\
3 \\
3 \\
2 \\
7\end{array}$ & $\begin{array}{l}\text { 43174. } 90 \\
43196.52 \\
43200.36 \\
43221.92 \\
43227.78\end{array}$ & $\begin{array}{l}a{ }^{5} \mathrm{~F}_{2}-442(1) \\
b{ }^{3} \mathrm{~F}_{4}-616(3) \\
a{ }^{5} \mathrm{~F}_{2}-442(2)\end{array}$ & $\begin{array}{l}(\mathbf{0 . 0 0 0}, 0.750) 0.253,0.998, \mathbf{1 . 7 5 1} \\
(\mathbf{0 . 0 0 0 ,} 0.307,0.621,0.940) 1.554,1.847,2.172 \\
(0.223, \mathbf{0 . 5 0 7}) 0.752, \mathbf{1 . 0 0 8}, \mathbf{1 . 2 5 1}, 1.481\end{array}$ \\
\hline $\begin{array}{l}\text { 11. } 46 \\
\text { 11. } 20 \\
\text { 08. } 93 \\
\text { 08. } 63 \\
\text { 08. } 51\end{array}$ & $\begin{array}{l}1 \\
5 \\
1 \\
2 \\
4\end{array}$ & $\begin{array}{l}\text { 43249. } 34 \\
\text { 43254. } 28 \\
\text { 43296. } 71 \\
\text { 43302. } 41 \\
\text { 43304. } 74\end{array}$ & $\begin{array}{l}a{ }^{5} \mathrm{D}_{2}-577(2) \\
a{ }^{3} \mathrm{P}_{2}-489(3)\end{array}$ & $(0.000) 1.207 A$ \\
\hline
\end{tabular}


TABLE 1. Wavelengths, term combinations, and Zeeman effects of Ta II-Continued

\begin{tabular}{|c|c|c|c|c|}
\hline $\begin{array}{c}\text { Wave } \\
\text { length } \\
\lambda \text { air }\end{array}$ & $\begin{array}{c}\text { Intensity } \\
\text { and } \\
\text { notes }\end{array}$ & $\begin{array}{l}\text { Wave } \\
\text { number }\end{array}$ & $\underset{\text { Term }}{\text { combination }}$ & Zeeman pattern \\
\hline 2308. 16 & 3 & 43311.15 & $\left\{\begin{array}{l}a^{3}{ }^{3} \mathrm{~F}_{2}-464(2) \\
a^{3} \mathrm{D}_{2}-604(1)\end{array}\right.$ & $(\mathbf{0 . 0 0 0}, 0.545) 0.660,1.192, \mathbf{1 . 7 4 9}$ \\
\hline 06. 86 & 3 & 43335. 62 & $\left\{\begin{array}{l}a{ }^{3} \mathrm{P}_{1}-486(2) \\
a^{5} \mathrm{~F}_{5}-495(5)\end{array}\right.$ & $(\mathbf{0 . 0 0 0}, 0.409) \mathbf{0 . 7 2 0}, 1.129,1.517$ \\
\hline 05. 49 & 1 & 43361. 38 & $a^{3} \mathrm{G}_{3}-551(3)$ & \\
\hline 04.75 & 3 & 43375. 30 & $a^{3} \mathrm{H}_{6}-613(5)$ & \\
\hline 03.48 & 6 & 43399. 14 & $a^{5} \mathrm{~F}_{2}-444(3)$ & $(0.000 w) 0.879 w$ \\
\hline 03. 31 & 1 & 43402. 42 & $a^{3} \mathrm{H}_{5}-615(6)$ & \\
\hline 02.92 & 6 & 43409. 69 & $a^{5} \mathrm{~F}_{4}-478(3)$ & $(0.000 D) 1.721 B$ \\
\hline 02. 39 & 1 & 43419. 76 & & \\
\hline 02. 23 & 6 & 43422. 74 & $\left\{\begin{array}{l}a^{3} \mathrm{H}_{5}-616(6) \\
a^{3} \mathrm{P}_{0}-490(2)\end{array}\right.$ & $(0.000 w) 1.330$ \\
\hline 01.47 & 8 & 43437. 04 & $a^{3} \mathrm{G}_{4}-561(4)$ & $(1.082 B) 0.240,0.499,0.752, \mathbf{1 . 0 0 8}, \mathbf{1 . 2 5 0}, 1.522,1.759,2.012$ \\
\hline 01.22 & 1 & 43441. 84 & $a^{5} \mathrm{~F}_{4}-478(5)$ & \\
\hline 01.07 & 2 & 43444. 63 & & \\
\hline 01.02 & 1 & 43445.61 & $a^{3} \mathrm{P}_{1}-487(1)$ & \\
\hline 00.27 & 2 & 43459. 72 & $b^{3} \mathrm{~F}_{3}-580(4)$ & $(0.000 w) 1.115 w$ \\
\hline 2298.95 & 6 & 43484.73 & & $(0.000 w) 0.639 A$ \\
\hline 98. 39 & 1 & 43495. 32 & $b{ }^{3} \mathrm{P}_{1}-608(2)$ & $(0.000) 1.114$ \\
\hline 98. 12 & 8 & 43500.39 & $a^{1} \mathrm{D}_{2}-570(3)$ & $(\mathbf{0 . 0 0 0}, 0.295,0.570) 1.093,1.378,1.643, \mathbf{1 . 8 9 9}$ \\
\hline 97. 63 & 2 & 43509. 67 & & (0.000) 1.104 \\
\hline 96. 81 & 40 & 43525. 20 & & $(0.00 w) 0.99 w$ \\
\hline 96. 68 & 2 & 43527. 70 & $c{ }^{3} \mathrm{~F}_{4}-666(5)$ & $(0.384 w) 1.066 D$ \\
\hline 95.55 & 1 & 43549. 11 & $c{ }^{3} \mathrm{P}_{0}-669(1)$ & $(0.000) 0.000,1.220$ \\
\hline 95. 22 & 4 & 43555. 48 & & $(0.000 w) 0.895 w$ \\
\hline 93. 77 & 1 & 43582. 92 & $a{ }^{5} \mathrm{P}_{3}-560(2)$ & \\
\hline $\begin{array}{l}92.56 \\
02 \\
02\end{array}$ & $\begin{array}{r}15 \\
4\end{array}$ & 43605. 94 & $a^{3} \mathrm{H}_{6}-615(6)$ & $(0.362 w) 1.138 D$ \\
\hline & & 43613.98 & $a^{3} \mathrm{G}_{3}-553(4)$ & $(0.000 D) 1.297 \mathrm{~A}$ \\
\hline 91.50 & 1 & 43626. 15 & $a^{5} \mathrm{D}_{3}-593(2)$ & \\
\hline 91. 47 & 1 & 43626.64 & $a^{3} \mathrm{H}_{6}-616(6)$ & \\
\hline 91. 00 & 1 & 43635. 61 & $\left\{\begin{array}{l}a^{1} \mathrm{D}_{2}-571(1) \\
b^{1} \mathrm{D}_{2}-669(1)\end{array}\right.$ & $(\mathbf{0 . 0 0 0}, 0.628) 0.490, \ldots$ \\
\hline 90. 46 & 4 & 43645. 93 & $a^{3} \mathrm{G}_{4}-563(3)$ & \\
\hline 89. 93 & 3 & 43656. 02 & & \\
\hline 89. 43 & 2 & 43665.53 & & \\
\hline 89. 18 & $1 \overline{5}$ & 43670.28 & $a^{3} \mathrm{~F}_{2}-468(2)$ & $(0.342, \mathbf{0 . 7 2 3}) \quad 0.389, \mathbf{0 . 7 3 9}, \mathbf{1 . 0 9 7}, 1.447$ \\
\hline $\begin{array}{l}\text { 88. } 88 \\
87.28\end{array}$ & $\begin{array}{r}3 \\
12\end{array}$ & 43676. 06 & $a{ }^{5} \mathrm{P}_{2}-555(2)$ & \\
\hline 87. 01 & $\begin{array}{r}12 \\
1\end{array}$ & $\begin{array}{l}43706.57 \\
43711.73\end{array}$ & $a^{5} \mathrm{P}_{3}-561(4)$ & $(\mathbf{0 . 0 0 0}, 0.319,0.664,1.023) \mathbf{0 . 2 7 7}, 0.594,0.926,1.268,1.572$ \\
\hline 86. 60 & 3 & 43719.59 & $a^{3} \mathrm{~F}_{4}-534(3)$ & $(0.000 w) 1.214$ \\
\hline 85. 27 & 20 & 43744. 97 & $a^{5} \mathrm{~F}_{3}-463(2)$ & $(0.000 w) 1.171 \mathrm{~A}$ \\
\hline 85. 02 & 7 & 43749.85 & $a^{3} \mathrm{P}_{1}-490(2)$ & $(\mathbf{0 . 0 0 0}, 0.257) \quad \mathbf{1 . 0 4 8}, 1.266,1.528$ \\
\hline 83. 67 & 2 & 43775.58 & $b^{3} \mathrm{~F}_{2}-534(3)$ & $(0.00) 1.40$ \\
\hline 83. 40 & 1 & 43780.91 & $b{ }^{3} \mathrm{H}_{4}-682(5)$ & \\
\hline 83. 22 & 8 & 43784. 32 & $a^{3} \mathrm{G}_{3}-555(2)$ & $(0.000) 0.706 A$ \\
\hline 83. 07 & 1 & 43787. 16 & & \\
\hline 82. 21 & 3 & 43803. 60 & $a^{5} \mathrm{~F}_{2}-448(3)$ & $(\mathbf{0 . 0 0 0}, 0.271,0.518) 1.014,1.264,1.519, \mathbf{1 . 7 6 0}$ \\
\hline 81.54 & 4 & 43816. 44 & $a^{3} \mathrm{G}_{4}-565(5)$ & $(0.000,0.912 w)$ \\
\hline & 3 & 43820.71 & $a^{5} \mathrm{P}_{1}-545(2)$ & $(0.000) 1.015$ \\
\hline 80.58 & 5 & 43834. 92 & & $(0.00 w) 1.27 w$ \\
\hline 78. 92 & 1 & 43866. 91 & $b{ }^{3} \mathrm{H}_{4}-682(3)$ & \\
\hline 78. 33 & 2 & 43878. 21 & $a^{3} \mathrm{P}_{2}-495(3)$ & $(0.00) 1.25$ \\
\hline 78. 11 & $\overline{2}$ & 43882.52 & & $(0.00) 1.18$ \\
\hline 75. 64 & 10 & 43930. 11 & $a^{3} \mathrm{H}_{5}-621(4)$ & $(0.00) 1.19$ \\
\hline 74. 61 & 2 & 43950.08 & & \\
\hline $\begin{array}{l}74.37 \\
72.72\end{array}$ & $\begin{array}{l}2 \\
1\end{array}$ & $\begin{array}{l}43954.66 \\
43986.55\end{array}$ & $\begin{array}{l}b^{3} \mathrm{~F}_{2}-536(2) \\
a^{3} \mathrm{H}_{4}-598(5)\end{array}$ & $(0.58) 1.16$ \\
\hline 72.59 & 20 & 43989. 02 & $\left\{\begin{array}{l}a{ }^{3} \mathrm{P}_{2}-496(3) \\
a^{3} \mathrm{~F}_{2}-471(3)\end{array}\right.$ & $(\mathbf{0 . 0 0}, 0.65) \quad 0.68,1.33$ \\
\hline 71. 86 & 15 & 44003. 24 & $\begin{cases}a^{5} \mathrm{~F}_{3}-466(4) \\
a^{5} \mathrm{P}_{2}-558(1)\end{cases}$ & $(0.00) 1.16$ \\
\hline
\end{tabular}


TABLE 1. Wavelengths, term combinations, and Zeeman effects of Ta II-Continued

\begin{tabular}{|c|c|c|c|c|}
\hline $\begin{array}{l}\text { Wave } \\
\text { length } \\
\text { 入air }\end{array}$ & $\begin{array}{l}\text { Intensity } \\
\text { and } \\
\text { notes }\end{array}$ & $\begin{array}{l}\text { Wave } \\
\text { number }\end{array}$ & $\begin{array}{c}\text { Term } \\
\text { combination }\end{array}$ & Zeeman pattern \\
\hline $\begin{array}{r}\text { 2271. } 27 \\
\text { 71. } 05 \\
\text { 70. } 90 \\
\text { 69. } 56 \\
\text { 69. } 21\end{array}$ & $\begin{array}{l}1 \\
2 \\
4 \\
5 \\
4\end{array}$ & $\begin{array}{l}44014.63 \\
44018.97 \\
44021.76 \\
44047.73 \\
44054.66\end{array}$ & $\begin{array}{l}a^{3} \mathrm{H}_{5}-622(5) \\
a^{3} \mathrm{G}_{4}-567(4) \\
a^{5} \mathrm{~F}_{4}-484(4)\end{array}$ & $\begin{array}{ll}(0.00) & 1.09 \\
(0.00) & 1.09 \\
(0.63) & 1.15 w \\
(0.00 w) & 1.29\end{array}$ \\
\hline $\begin{array}{l}\text { 67. } 50 \\
\text { 67. } 27 \\
\text { 65. } 94 \\
\text { 64. } 45 \\
63.98\end{array}$ & $\begin{array}{l}2 \\
4 \\
1 \\
2 \\
3\end{array}$ & $\begin{array}{l}\text { 44087. } 86 \\
\text { 44092. } 31 \\
\text { 44118. } 23 \\
\text { 44127. } 14 \\
\text { 44156. } 38\end{array}$ & $a^{3} \mathrm{G}_{3}-558(2)$ & $\begin{array}{l}(0.00 w) 0.70 w \\
(0.00 w) 0.82 w \\
(0.00) 1.04\end{array}$ \\
\hline $\begin{array}{l}62.29 \\
61.62 \\
61.43 \\
60.57 \\
60.26\end{array}$ & $\begin{array}{r}25 \\
12 \\
20 \\
5 \\
3\end{array}$ & $\begin{array}{l}44189.28 \\
44202.45 \\
44206.09 \\
44222.90 \\
44229.09\end{array}$ & $\begin{array}{l}a{ }^{5} \mathrm{~F}_{3}-468(3) \\
a{ }^{5} \mathrm{~F}_{2}-452(1) \\
a \\
{ }^{5} \mathrm{~F}_{1}-442(1) \\
a^{3} \mathrm{H}_{6}-622(5) \\
a^{3} \mathrm{P}_{2}-498(1)\end{array}$ & $\begin{array}{l}(0.00) 1.15 \\
(\mathbf{0 . 0 0}, 0.48) \quad 0.48,1.00 \\
(0.00) 1.43 A \\
(\mathbf{0 . 0 0}, 0.67) 0.71\end{array}$ \\
\hline $\begin{array}{l}59.59 \\
59.12 \\
58.74 \\
57.55 \\
57.11\end{array}$ & $\begin{array}{l}5 \\
3 \\
5 \\
3 \\
1\end{array}$ & $\begin{array}{l}44242.18 \\
44251.40 \\
44258.83 \\
44282.15 \\
44290.73\end{array}$ & $\begin{array}{l}a{ }^{3} \mathrm{~F}_{3}-510(3) \\
a^{3} \mathrm{G}_{3}-560(2) \\
a^{5} \mathrm{~F}_{1}-442(2)\end{array}$ & $\begin{array}{lll}(0.18) & 1.13 & \\
(0.00) & 0.81 \\
(\mathbf{0 . 0 0}, & 1.24) & 0.00,1.24,2.53 \\
(0.41) & 1.09\end{array}$ \\
\hline $\begin{array}{l}56.53 \\
55.77 \\
55.60 \\
54.88 \\
53.47\end{array}$ & $\begin{array}{r}12 \\
20 \\
2 \\
4 \\
2\end{array}$ & $\begin{array}{l}44302.07 \\
44317.08 \\
44320.30 \\
44334.45 \\
44362.24\end{array}$ & $\begin{array}{lll}a & { }^{5} \mathrm{P}_{3}-567(4) \\
a & { }^{5} \mathrm{~F}_{5}-505(5) \\
a & { }^{3} \mathrm{~F}_{2}-475(2)\end{array}$ & $\begin{array}{l}(0.00) 1.20 \\
(0.00 W) 0.00 W \\
(1.09) 1.38\end{array}$ \\
\hline $\begin{array}{l}53.26 \\
52.80 \\
52.06 \\
50.78 \\
49.79\end{array}$ & $\begin{array}{r}3 \\
3 \\
2 \\
15 \\
10\end{array}$ & $\begin{array}{l}44366.34 \\
44375.46 \\
44390.02 \\
44415.38 \\
44434.74\end{array}$ & $\begin{array}{l}a{ }^{3} \mathrm{~F}_{3}-511(2) \\
a^{3} \mathrm{G}_{3}-561(4) \\
a^{3} \mathrm{H}_{4}-602(3) \\
a{ }^{5} \mathrm{~F}_{2}-454(2) \\
a{ }^{5} \mathrm{~F}_{1}-444(0)\end{array}$ & $\begin{array}{l}(0.00 w) 0.78 \\
(0.00 w) 1.42 w \\
(0.36) 1.13 \\
(0.00) 0.00\end{array}$ \\
\hline $\begin{array}{l}\text { 48. } 50 \\
47.84 \\
47.40 \\
46.16 \\
43.87\end{array}$ & $\begin{array}{l}5 \\
2 \\
1 \\
2 \\
6\end{array}$ & $\begin{array}{l}44460.37 \\
44473.38 \\
44482.11 \\
44506.72 \\
44552.08\end{array}$ & $\begin{array}{c}a{ }^{3} \mathrm{~F}_{4}-542(4) \\
\left\{\begin{array}{l}a{ }^{3} \mathrm{D}_{3}-630(2) \\
a^{5} \mathrm{~F}_{5}-506(6) \\
a^{5} \mathrm{P}_{3}-569(4)\end{array}\right.\end{array}$ & $(0.00 w) 0.00 w$ \\
\hline $\begin{array}{l}\text { 43. } 47 \\
\text { 42. } 68 \\
\text { 41. } 72\end{array}$ & $\begin{array}{l}2 \\
5 \\
2\end{array}$ & $\begin{array}{l}44559.96 \\
44575.74 \\
44594.86\end{array}$ & $\begin{array}{l}b{ }^{3} \mathrm{P}_{2}-630(2) \\
a^{5} \mathrm{P}_{2}-564(3) \\
a^{5} \mathrm{D}_{0}-571(1)\end{array}$ & $(0.00 w) 1.01 w$ \\
\hline $\begin{array}{l}39.48 \\
39.01\end{array}$ & $\begin{array}{r}20 \\
5\end{array}$ & $\begin{array}{l}\text { 44639. } 23 \\
44648.80\end{array}$ & 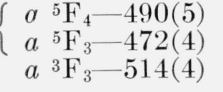 & 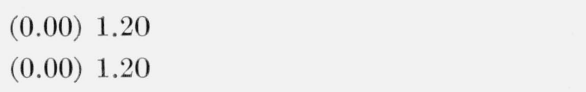 \\
\hline $\begin{array}{l}38.60 \\
37.46 \\
36.29 \\
35.94 \\
35.69\end{array}$ & $\begin{array}{l}1 \\
2 \\
4 \\
3 \\
3\end{array}$ & $\begin{array}{l}44657.01 \\
44679.62 \\
44702.96 \\
44709.97 \\
44714.97\end{array}$ & 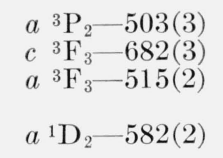 & $(0.00) 0.84$ \\
\hline $\begin{array}{l}33.89 \\
32.26 \\
31.46 \\
30.86 \\
30.69\end{array}$ & $\begin{array}{l}4 \\
1 \\
1 \\
3 \\
1\end{array}$ & $\begin{array}{l}44751.08 \\
44783.81 \\
44799.75 \\
44811.88 \\
44815.25\end{array}$ & $\begin{array}{l}a{ }^{5} \mathrm{D}_{1}-582(2) \\
a{ }^{5} \mathrm{P}_{1}-555(1)\end{array}$ & \\
\hline $\begin{array}{l}29.90 \\
29.30 \\
29.03 \\
27.85 \\
27.23\end{array}$ & $\begin{array}{r}1 \\
1 \\
1 \\
50 \\
10\end{array}$ & $\begin{array}{l}\text { 44831. } 11 \\
44843.27 \\
44848.62 \\
44872.37 \\
44884.82\end{array}$ & $\begin{array}{l}b{ }^{3} \mathrm{~F}_{2}-545(2) \\
a{ }^{5} \mathrm{~F}_{3}-475(2) \\
a{ }^{5} \mathrm{D}_{4}-621(4)\end{array}$ & $(0.00) 1.22$ \\
\hline
\end{tabular}


TABLE 1. Wavelengths, term combinations, and Zeeman effects of Ta II-Continued

\begin{tabular}{|c|c|c|c|c|}
\hline $\begin{array}{l}\text { Wave } \\
\text { length } \\
\text { 入air }\end{array}$ & $\begin{array}{l}\text { Intensity } \\
\text { and } \\
\text { notes }\end{array}$ & $\begin{array}{l}\text { Wave } \\
\text { number }\end{array}$ & $\begin{array}{c}\text { Term } \\
\text { combination }\end{array}$ & Zeeman pattern \\
\hline $\begin{array}{r}2226.52 \\
25.42 \\
24.91 \\
23.56 \\
23.31\end{array}$ & $\begin{array}{r}15 \\
3 \\
1 \\
1 \\
10\end{array}$ & $\begin{array}{l}\text { 44899. } 18 \\
44921.33 \\
44931.62 \\
44958.94 \\
44963.91\end{array}$ & $\begin{array}{l}a^{3} \mathrm{D}_{3}-634(4) \\
b^{3} \mathrm{~F}_{2}-546(3)\end{array}$ & $\begin{array}{l}(0.00 w) 1.05,1.72 \\
(0.00 w) 1.55 w\end{array}$ \\
\hline $\begin{array}{l}22.80 \\
22.56\end{array}$ & $\begin{array}{l}1 \\
5\end{array}$ & $\begin{array}{l}44974.31 \\
44979.23\end{array}$ & & \\
\hline 22. 22 & 4 & 44986.05 & $\left\{\begin{array}{l}a^{3} \mathrm{G}_{3}-567(4) \\
a^{3} \mathrm{~F}_{2}-481(2)\end{array}\right.$ & \\
\hline $\begin{array}{l}\text { 20. } 70 \\
\text { 19. } 41\end{array}$ & $\begin{array}{l}15 \\
10\end{array}$ & $\begin{array}{l}\text { 45016. } 79 \\
\text { 45043. } 04\end{array}$ & $a^{3} \mathrm{~F}_{2}-482(2)$ & $(1.42) \ldots$ \\
\hline $\begin{array}{l}\text { 18. } 93 \\
18.39 \\
17.84 \\
16.09 \\
15.58\end{array}$ & $\begin{array}{r}1 \\
6 \\
15 \\
4 \\
20\end{array}$ & $\begin{array}{l}45052.74 \\
45063.75 \\
45074.80 \\
45110.43 \\
45120.77\end{array}$ & $a^{5} \mathrm{~F}_{4}-495(3)$ & $\begin{array}{l}(0.00) 1.07 \\
(0.00) 1.41\end{array}$ \\
\hline $\begin{array}{l}\text { 14. } 53 \\
\text { 14. } 33 \\
\text { 13. } 39 \\
\text { 12. } 42 \\
\text { 11. } 96\end{array}$ & $\begin{array}{r}25 \\
8 \\
1 \\
20 \\
1\end{array}$ & $\begin{array}{l}45142.21 \\
45146.36 \\
45165.52 \\
45185.40 \\
45194.61\end{array}$ & $\begin{array}{l}a \\
a{ }^{5} \mathrm{P}_{1}-558(2) \\
a^{5} \mathrm{P}_{1}-558(1) \\
a^{5} \mathrm{P}_{2}-570(3)\end{array}$ & $\begin{array}{l}(0.00 w) 1.30 w \\
(0.00) 0.00 w \\
(0.00) 1.42\end{array}$ \\
\hline $\begin{array}{l}\text { 11. } 66 \\
\text { 10. } 20 \\
\text { 10. } 04 \\
\text { 09. } 03 \\
\text { 07. } 99\end{array}$ & $\begin{array}{l}2 \\
6 \\
5 \\
3 \\
2\end{array}$ & $\begin{array}{l}45200.74 \\
45230.78 \\
45234.01 \\
45254.05 \\
45276.00\end{array}$ & $\begin{array}{l}a^{5} \mathrm{~F}_{4}-496(3) \\
a{ }^{5} \mathrm{~F}_{1}-452(1) \\
a^{3} \mathrm{H}_{4}-611(4)\end{array}$ & $(1.45) \quad 0.00,1.43$ \\
\hline $\begin{array}{l}\text { 07. } 64 \\
07.36 \\
\text { 07. } 13 \\
07.04 \\
06.54\end{array}$ & $\begin{array}{r}3 \\
3 \\
10 \\
5 \\
4\end{array}$ & $\begin{array}{l}45283.16 \\
45288.78 \\
45293.56 \\
45295.43 \\
45305.67\end{array}$ & $\begin{array}{l}a^{3} \mathrm{G}_{3}-570(3) \\
a{ }^{5} \mathrm{P}_{1}-560(2)\end{array}$ & \\
\hline $\begin{array}{l}05.86 \\
05.49\end{array}$ & $\begin{array}{l}1 \\
1\end{array}$ & $\begin{array}{l}45319.76 \\
45327.34\end{array}$ & $\begin{array}{l}a{ }^{5} \mathrm{P}_{2}-571(1) \\
b^{3} \mathrm{~F}_{4}-638(4)\end{array}$ & \\
\hline 04. 09 & 5 & 45356.01 & $\left\{\begin{array}{l}a \\
{ }^{5} \mathrm{P}_{3}-577(2) \\
a^{5} \mathrm{~F}_{2}-463(2)\end{array}\right.$ & \\
\hline $\begin{array}{l}02.83 \\
02.28\end{array}$ & $\begin{array}{l}1 \\
4\end{array}$ & $\begin{array}{l}\text { 45381. } 95 \\
45393.24\end{array}$ & $a^{5} \mathrm{~F}_{4}-551(3)$ & \\
\hline $\begin{array}{r}01.80 \\
01.15 \\
00.43\end{array}$ & $\begin{array}{r}3 \\
12 \\
8 \\
25\end{array}$ & $\begin{array}{l}45403.22 \\
45416.54 \\
45431.49 \\
45447.26\end{array}$ & $a^{3} \mathrm{P}_{2}-510(3)$ & $(0.00) 1.03$ \\
\hline $\begin{array}{r}2199.67 \\
97.77\end{array}$ & $\begin{array}{r}25 \\
1\end{array}$ & $\begin{array}{l}\text { 45447. } 26 \\
45486.46\end{array}$ & $\left\{\begin{array}{l}a{ }^{5} \mathrm{~F}_{1}-454(2) \\
a^{1} \mathrm{G}_{4}-596(5) \\
a^{3} \mathrm{~F}_{2}-486(2)\end{array}\right.$ & $(\mathbf{0 . 0 0}, 1.12) 1.29, \mathbf{2 . 3 9}$ \\
\hline $\begin{array}{l}\text { 96. } 30 \\
96.05 \\
95.23 \\
93.88 \\
93.22\end{array}$ & $\begin{array}{r}3 \\
50 \\
1 \\
25 \\
15\end{array}$ & $\begin{array}{l}45516.90 \\
45522.04 \\
45539.09 \\
45567.19 \\
45580.90\end{array}$ & $\begin{array}{l}a^{3} \mathrm{H}_{5}-637(5) \\
a \\
{ }^{5} \mathrm{~F}_{4}-499(4) \\
a \\
{ }^{3} \mathrm{P}_{2}-511(2) \\
a \\
{ }^{5} \mathrm{~F}_{5}-517(5) \\
a^{5} \mathrm{~F}_{3}-482(2)\end{array}$ & (0.41) 1.40 \\
\hline $\begin{array}{l}\text { 92. } 47 \\
91.99 \\
90.97\end{array}$ & $\begin{array}{l}2 \\
4 \\
2\end{array}$ & $\begin{array}{l}45596.34 \\
45606.45 \\
45627.66\end{array}$ & & \\
\hline $\begin{array}{l}90.62 \\
89.40\end{array}$ & $\begin{array}{l}2 \\
1\end{array}$ & $\begin{array}{l}45634.80 \\
45660.38\end{array}$ & $\left\{\begin{array}{l}a^{3} \mathrm{H}_{5}-638(4) \\
a^{3} \mathrm{~F}_{4}-553(4) \\
b^{3} \mathrm{~F}_{3}-602(3)\end{array}\right.$ & \\
\hline $\begin{array}{l}\text { 88. } 48 \\
86.44 \\
86.26 \\
85.49 \\
85.30\end{array}$ & $\begin{array}{l}8 \\
4 \\
2 \\
2 \\
2\end{array}$ & $\begin{array}{l}45679.57 \\
45722.06 \\
45725.91 \\
45742.00 \\
45746.10\end{array}$ & $\begin{array}{l}a^{3} \mathrm{G}_{5}-585(5) \\
a^{5} \mathrm{D}_{2}-602(3)\end{array}$ & \\
\hline
\end{tabular}


TABLE 1. Wavelengths, term combinations, and Zeeman effects of Ta II-Continued

\begin{tabular}{|c|c|c|c|c|}
\hline $\begin{array}{l}\text { Wave } \\
\text { length } \\
\lambda \text { air }\end{array}$ & $\begin{array}{l}\text { Intensity } \\
\text { and } \\
\text { notes }\end{array}$ & $\begin{array}{l}\text { Wave } \\
\text { number }\end{array}$ & $\begin{array}{c}\text { Term } \\
\text { combination }\end{array}$ & Zeeman pattern \\
\hline $\begin{array}{r}2185.15 \\
84.68 \\
83.44 \\
83.11 \\
82.72\end{array}$ & $\begin{array}{r}2 \\
8 \\
2 \\
3 \\
50\end{array}$ & $\begin{array}{l}45759.11 \\
45758.98 \\
45784.96 \\
45791.80 \\
45800.10\end{array}$ & $\begin{array}{l}a^{3} \mathrm{~F}_{3}-525(3) \\
a^{3} \mathrm{~F}_{4}-555(5) \\
a^{1} \mathrm{D}_{2}-593(2) \\
a^{5} \mathrm{~F}_{2}-468(3)\end{array}$ & $(0.00) 1.53$ \\
\hline $\begin{array}{l}\text { 81. } 80 \\
\text { 81. } 38\end{array}$ & $\begin{array}{l}4 \\
1\end{array}$ & $\begin{array}{l}45819.39 \\
45828.15\end{array}$ & $\begin{array}{l}a^{5} \mathrm{~F}_{2}-468(2) \\
a^{5} \mathrm{~F}_{3}-484(4)\end{array}$ & \\
\hline 80.87 & 8 & 45838.84 & $\left\{\begin{array}{rr}a^{3} \mathrm{H}_{6} & 638(5) \\
a^{5} \mathrm{P}_{3}-582(2)\end{array}\right.$ & \\
\hline $\begin{array}{l}\text { 80. } 68 \\
80.18\end{array}$ & $\begin{array}{r}10 \\
3\end{array}$ & $\begin{array}{l}45842.90 \\
45853.33\end{array}$ & $\begin{array}{l}a^{3} \mathrm{H}_{4}-616(3) \\
b^{3} \mathrm{~F}_{2}-555(3)\end{array}$ & \\
\hline $\begin{array}{l}79.81 \\
79.55 \\
79.06 \\
79.98 \\
78.03\end{array}$ & $\begin{array}{r}3 \\
25 \\
2 \\
1 \\
4\end{array}$ & $\begin{array}{l}45861.20 \\
45866.62 \\
45876.88 \\
45878.58 \\
45898.63\end{array}$ & $\begin{array}{l}b{ }^{3} \mathrm{~F}_{2}-555(2) \\
a^{3} \mathrm{P}_{1}-511(2) \\
a^{5} \mathrm{D}_{1}-593(2) \\
a^{5} \mathrm{~F}_{4}-503(3)\end{array}$ & \\
\hline $\begin{array}{l}77.97 \\
77.20 \\
74.72 \\
73.42 \\
73.05\end{array}$ & $\begin{array}{r}25 \\
1 \\
3 \\
2 \\
5\end{array}$ & $\begin{array}{l}45899.94 \\
45916.06 \\
45968.46 \\
45996.02 \\
46003.81\end{array}$ & $\begin{array}{l}a{ }^{3} \mathrm{~F}_{2}-490(2) \\
a^{5} \mathrm{P}_{2}-577(2) \\
a^{5} \mathrm{D}_{3}-616(3) \\
a^{3} \mathrm{P}_{1}-513(1)\end{array}$ & \\
\hline $\begin{array}{l}72.85 \\
72.15\end{array}$ & $\begin{array}{r}7 \\
15\end{array}$ & $\begin{array}{l}46008.08 \\
46022.95\end{array}$ & $\begin{array}{ll}b & { }^{3} \mathrm{P}_{0}-622(1) \\
a & { }^{1} \mathrm{~F}_{3}-708(2)\end{array}$ & \\
\hline 72.08 & 7 & 46024.39 & $\left\{\begin{array}{l}a^{5} \mathrm{~F}_{3}-486(2) \\
a^{3} \mathrm{G}_{3}-577(2)\end{array}\right.$ & \\
\hline $\begin{array}{l}71.02 \\
70.65\end{array}$ & $\begin{array}{l}1 \\
2\end{array}$ & $\begin{array}{l}\text { 46046. } 86 \\
46054.71\end{array}$ & $\begin{array}{c}2 \\
\text { o }\end{array}$ & \\
\hline $\begin{array}{l}69.15 \\
67.02 \\
66.76 \\
66.32 \\
65.45\end{array}$ & $\begin{array}{r}10 \\
1 \\
3 \\
10 \\
3\end{array}$ & $\begin{array}{l}\text { 46086. } 55 \\
46131.85 \\
46137.27 \\
46146.86 \\
46165.29\end{array}$ & $a^{5} \mathrm{~F}_{2}-471(3)$ & \\
\hline $\begin{array}{l}65.26 \\
65.00 \\
64.77 \\
64.67 \\
64.36\end{array}$ & $\begin{array}{r}3 \\
15 \\
1 \\
15 \\
5\end{array}$ & $\begin{array}{l}46169.34 \\
46174.92 \\
46179.79 \\
46181.86 \\
46188.45\end{array}$ & $\begin{array}{l}a{ }^{5} \mathrm{~F}_{1}-461(1) \\
a^{3} \mathrm{~F}_{3}-530(2) \\
b{ }^{3} \mathrm{~F}_{2}-558(1)\end{array}$ & \\
\hline $\begin{array}{l}64.12 \\
63.65 \\
62.65 \\
62.45 \\
62.26\end{array}$ & $\begin{array}{l}1 \\
8 \\
5 \\
2 \\
7\end{array}$ & $\begin{array}{l}\text { 46193. } 66 \\
46203.65 \\
46225.05 \\
46229.33 \\
46233.35\end{array}$ & $\begin{array}{l}a{ }^{5} \mathrm{P}_{2}-580(1) \\
a{ }^{3} \mathrm{P}_{1}-515(2)\end{array}$ & \\
\hline $\begin{array}{l}62.04 \\
61.88 \\
61.82 \\
61.26 \\
60.79\end{array}$ & $\begin{array}{r}4 \\
10 \\
5 \\
7 \\
3\end{array}$ & $\begin{array}{l}46238.09 \\
46241.60 \\
46242.80 \\
46254.82 \\
46264.82\end{array}$ & $\begin{array}{l}a^{3} \mathrm{~F}_{4}-569(4) \\
a^{3} \mathrm{D}_{1}-608(2) \\
a^{5} \mathrm{D}_{4}-634(4) \\
a^{3} \mathrm{H}_{4}-621(4)\end{array}$ & \\
\hline $\begin{array}{l}60.36 \\
59.63 \\
58.84 \\
58.42 \\
58.19\end{array}$ & $\begin{array}{r}4 \\
6 \\
2 \\
25 \\
3\end{array}$ & $\begin{array}{l}46273.96 \\
46289.71 \\
46306.62 \\
46315.68 \\
46320.57\end{array}$ & $\begin{array}{l}b{ }^{3} \mathrm{~F}_{3}-608(2) \\
a{ }^{5} \mathrm{~F}_{5}-524(4) \\
a{ }^{5} \mathrm{~F}_{3}-489(3)\end{array}$ & \\
\hline $\begin{array}{l}58.09 \\
57.83 \\
57.66 \\
56.91 \\
56.65\end{array}$ & $\begin{array}{l}8 \\
4 \\
1 \\
6 \\
1\end{array}$ & $\begin{array}{ll}46322 . & 71 \\
46328.27 \\
46331.99 \\
46348.10 \\
46353.64\end{array}$ & $\begin{array}{l}b^{3} \mathrm{~F}_{2}-560(2) \\
a^{3} \mathrm{H}_{4}-622(5)\end{array}$ & \\
\hline
\end{tabular}


TABLE 1. Wavelengths, term combinations, and Zeeman effects of Ta $\mathrm{II}$-Continued

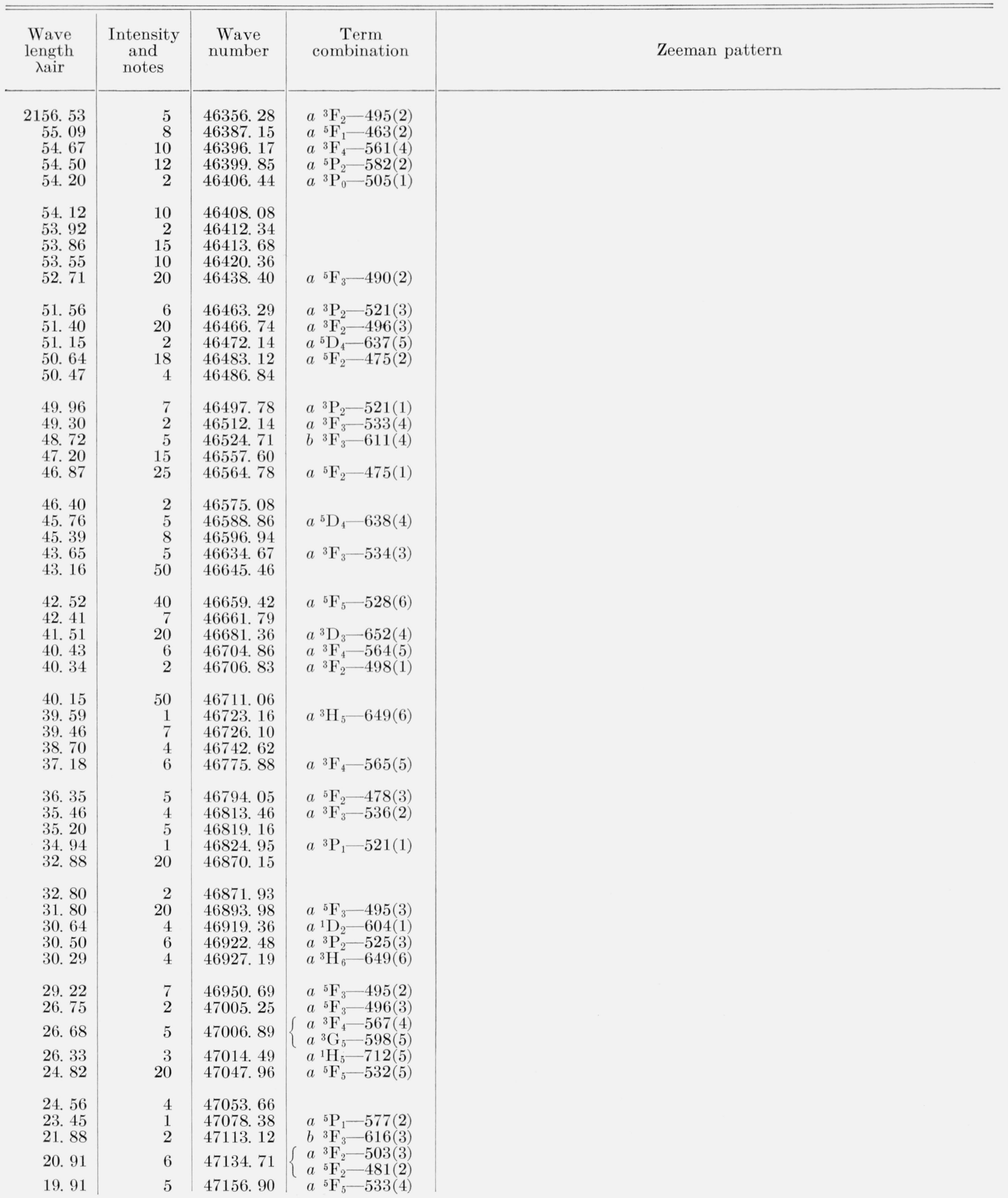


TABLE 1. Wavelengths, term combinations, and Zeeman effects of Ta II-Continued

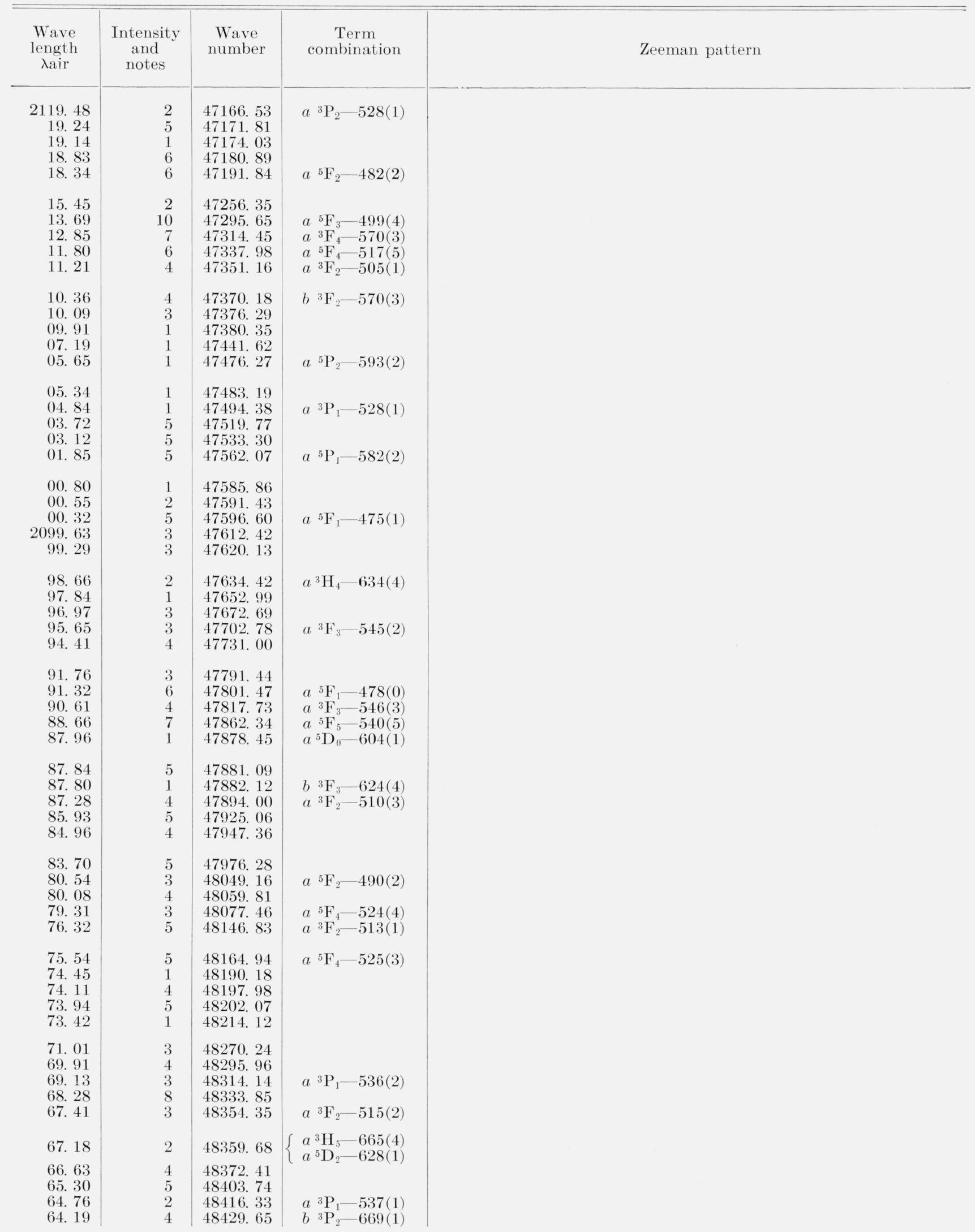


TABLE 1. Wavelengths, term combinations, and Zeeman effects of Ta II-Continued

\begin{tabular}{|c|c|c|c|c|}
\hline $\begin{array}{l}\text { Wave } \\
\text { length } \\
\text { xair }\end{array}$ & $\begin{array}{l}\text { Intensity } \\
\text { and } \\
\text { notes }\end{array}$ & $\begin{array}{l}\text { Wave } \\
\text { number }\end{array}$ & $\begin{array}{c}\text { Term } \\
\text { combination }\end{array}$ & Zeeman pattern \\
\hline $\begin{array}{r}2063.34 \\
61.99 \\
61.91 \\
60.99 \\
60.05\end{array}$ & $\begin{array}{r}5 \\
2 \\
10 \\
1 \\
2\end{array}$ & $\begin{array}{l}48449.55 \\
48481.25 \\
48483.13 \\
48504.84 \\
48527.01\end{array}$ & $\begin{array}{l}a{ }^{5} \mathrm{P}_{2}-603(3) \\
a{ }^{5} \mathrm{~F}_{2}-495(3) \\
a^{3} \mathrm{G}_{5}-613(5)\end{array}$ & \\
\hline $\begin{array}{l}\text { 59. } 08 \\
58.84 \\
58.59 \\
57.61 \\
56.78\end{array}$ & $\begin{array}{r}10 \\
2 \\
2 \\
2 \\
1\end{array}$ & $\begin{array}{l}48549.90 \\
48555.48 \\
48561.47 \\
48584.50 \\
48604.15\end{array}$ & $\begin{array}{lll}a & { }^{3} \mathrm{~F}_{3}-553(4) \\
a & { }^{5} \mathrm{~F}_{3}-511(2) \\
a^{5} & \mathrm{~F}_{2}-495(2) \\
b & { }^{3} \mathrm{~F}_{2}-582(2) \\
a^{5} & \mathrm{P}_{2}-604(1)\end{array}$ & \\
\hline $\begin{array}{l}\text { 55. } 76 \\
55.26 \\
53.84 \\
52.73 \\
51.54\end{array}$ & $\begin{array}{l}6 \\
1 \\
4 \\
2 \\
2\end{array}$ & $\begin{array}{l}48628.13 \\
48640.02 \\
48673.74 \\
48699.99 \\
48728.21\end{array}$ & $\begin{array}{l}a{ }^{3} \mathrm{H}_{6}-666(5) \\
a{ }^{3} \mathrm{P}_{0}-528(1)\end{array}$ & \\
\hline $\begin{array}{l}51.17 \\
50.28 \\
49.46 \\
47.40 \\
46.19\end{array}$ & $\begin{array}{l}1 \\
1 \\
1 \\
8 \\
2\end{array}$ & $\begin{array}{l}\text { 48737. } 05 \\
48758.13 \\
\text { 48777. } 68 \\
48826.90 \\
48855.60\end{array}$ & $\begin{array}{l}a^{1} \mathrm{D}_{2}-623(2) \\
a^{3} \mathrm{G}_{5}-616(6) \\
a^{3} \mathrm{~F}_{4}-585(5) \\
a^{5} \mathrm{~F}_{2}-498(1)\end{array}$ & \\
\hline $\begin{array}{l}44.66 \\
44.11 \\
43.16 \\
40.61 \\
40.01\end{array}$ & $\begin{array}{l}5 \\
2 \\
5 \\
5 \\
5\end{array}$ & $\begin{array}{l}48892.08 \\
48905.45 \\
48927.99 \\
48989.13 \\
49003.53\end{array}$ & $\begin{array}{l}a{ }^{5} \mathrm{~F}_{3}-515(2) \\
a{ }^{5} \mathrm{~F}_{4}-533(4) \\
a^{3} \mathrm{G}_{4}-616(3)\end{array}$ & \\
\hline $\begin{array}{l}38.10 \\
35.89 \\
34.19 \\
32.61 \\
32.36\end{array}$ & $\begin{array}{l}2 \\
3 \\
2 \\
1 \\
2\end{array}$ & $\begin{array}{l}49049.45 \\
49102.69 \\
49143.81 \\
49181.96 \\
49187.94\end{array}$ & $\begin{array}{l}a{ }^{5} \mathrm{~F}_{4}-534(3) \\
a^{3} \mathrm{G}_{3}-608(2) \\
a^{3} \mathrm{~F}_{3}-560(2)\end{array}$ & \\
\hline $\begin{array}{l}31.53 \\
31.17 \\
30.62 \\
30.25 \\
29.92\end{array}$ & $\begin{array}{l}2 \\
1 \\
1 \\
1 \\
1\end{array}$ & $\begin{array}{l}\text { 49208. } 06 \\
49216.77 \\
49230.20 \\
49239.20 \\
49247.05\end{array}$ & $b^{3} \mathrm{~F}_{3}-638(4)$ & \\
\hline $\begin{array}{l}28.40 \\
27.92 \\
27.12 \\
24.00 \\
23.20\end{array}$ & $\begin{array}{l}1 \\
4 \\
1 \\
4 \\
2\end{array}$ & $\begin{array}{l}49284.05 \\
49295.64 \\
49315.16 \\
49391.10 \\
49410.72\end{array}$ & $a^{3} \mathrm{G}_{4}-621(4)$ & \\
\hline $\begin{array}{l}22.66 \\
22.21 \\
20.74\end{array}$ & $\begin{array}{l}1 \\
1 \\
1\end{array}$ & $\begin{array}{l}49423.94 \\
49434.96 \\
49470.82\end{array}$ & $a^{3} \mathrm{P}_{2}-551(3)$ & \\
\hline 19.56 & 5 & 49499. 65 & $\left\{\begin{array}{l}a^{5} \mathrm{~F}_{2}-505(1) \\
a^{3} \mathrm{G}_{4}-622(5)\end{array}\right.$ & \\
\hline 19. 18 & 1 & 49509. 11 & $a^{5} \mathrm{D}_{3}-652(4)$ & \\
\hline $\begin{array}{l}17.71 \\
15.50 \\
14.66 \\
13.17 \\
09.19\end{array}$ & $\begin{array}{l}2 \\
2 \\
2 \\
6 \\
2\end{array}$ & $\begin{array}{l}49545.12 \\
49599.56 \\
49620.17 \\
49656.86 \\
49755.11\end{array}$ & & \\
\hline $\begin{array}{l}06.71 \\
05.86 \\
04.54 \\
03.92 \\
03.16\end{array}$ & $\begin{array}{l}6 \\
1 \\
4 \\
4 \\
1\end{array}$ & $\begin{array}{l}\text { 49816. } 60 \\
49837.83 \\
49870.56 \\
49885.92 \\
49904.84\end{array}$ & $a^{3} \mathrm{P}_{2}-555(3)$ & \\
\hline $\begin{array}{l}02.28 \\
01.81 \\
01.17 \\
00.73\end{array}$ & $\begin{array}{l}2 \\
5 \\
2 \\
4\end{array}$ & $\begin{array}{l}\text { 49926. } 82 \\
\text { 49938. } 72 \\
\text { 49954. } 51 \\
\text { 49965. } 47\end{array}$ & $\begin{array}{l}a^{3} \mathrm{G}_{3}-616(3) \\
a^{5} \mathrm{~F}_{3}-525(3)\end{array}$ & \\
\hline
\end{tabular}


TABle 2. Even terms of Ta II

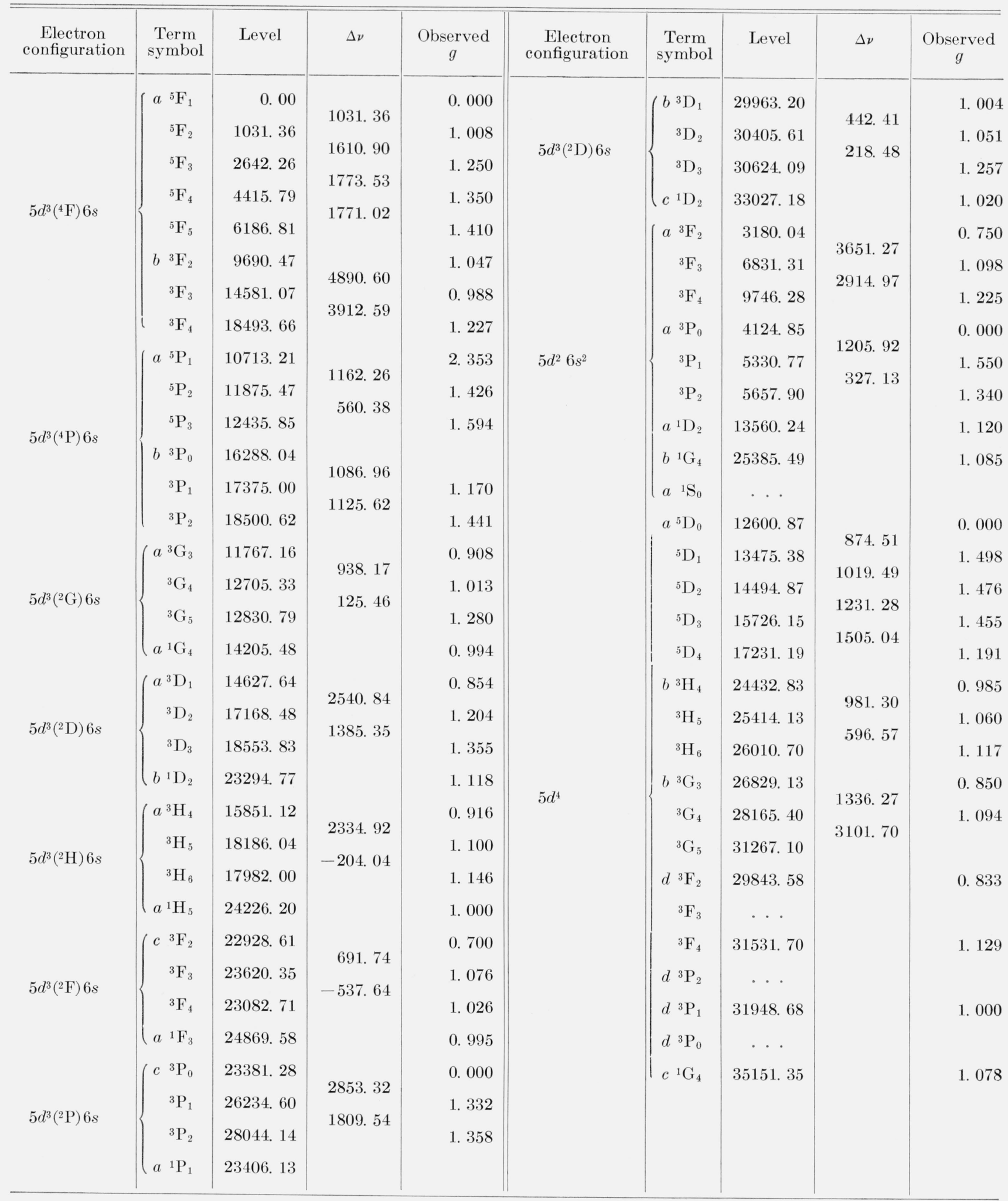


TABLE 3. Odd levels of Ta II

\begin{tabular}{|c|c|c|c|c|c|c|c|c|c|c|c|}
\hline Level & $J$ & $\underset{g}{\text { Observed }}$ & Level & $J$ & $\underset{g}{\text { Observed }}$ & Level & $J$ & $\begin{array}{c}\text { Observed } \\
g\end{array}$ & Level & $J$ & $\underset{g}{\text { Observed }}$ \\
\hline $\begin{array}{l}29256.87 \\
31212.13 \\
32318.44 \\
33706.47 \\
33715.27\end{array}$ & $\begin{array}{l}2 \\
1 \\
3 \\
1 \\
2\end{array}$ & $\begin{array}{l}0.976 \\
0.285 \\
0.823\end{array}$ & $\begin{array}{l}45233.91 \\
45446.85 \\
45466.97 \\
46174.60 \\
46286.90\end{array}$ & $\begin{array}{l}1 \\
2 \\
5 \\
1 \\
4\end{array}$ & $\begin{array}{l}\text { 1. } 458 \\
\text { 1. } 148 \\
\text { 1. } 090 \\
\text { 1. } 367\end{array}$ & $\begin{array}{l}51073.92 \\
51197.42 \\
51326.31 \\
51479.86 \\
51534.28\end{array}$ & $\begin{array}{l}3 \\
2 \\
1 \\
4 \\
2\end{array}$ & $\begin{array}{l}\text { 1. } 156 \\
\text { 1. } 359 \\
\text { 1. } 047 \\
\text { 1. } 095 \\
\text { 1. } 299\end{array}$ & $\begin{array}{l}57195.81 \\
57791.58 \\
58040.89 \\
58069.18 \\
58274.85\end{array}$ & $\begin{array}{l}1 \\
2 \\
4 \\
1 \\
2\end{array}$ & $\begin{array}{l}\text { 1. } 726 \\
\text { 1. } 050 \\
\text { 1. } 045 \\
\text { 1. } 147 \\
\text { 1. } 641\end{array}$ \\
\hline $\begin{array}{l}36112.97 \\
36177.10 \\
36763.70 \\
36987.71 \\
37230.75\end{array}$ & $\begin{array}{l}4 \\
2 \\
3 \\
1 \\
2\end{array}$ & $\begin{array}{l}\text { 1. } 180 \\
0.946 \\
\text { 1. } 169 \\
0.685 \\
0.623\end{array}$ & $\begin{array}{l}46295.03 \\
46387.16 \\
46491.44 \\
46645.70 \\
46776.78\end{array}$ & $\begin{array}{l}5 \\
2 \\
2 \\
4 \\
2\end{array}$ & $\begin{array}{l}\text { 1. } 200 \\
\text { 1. } 323 \\
\text { 1. } 060 \\
\text { 1. } 233 \\
0.773\end{array}$ & $\begin{array}{l}51753.70 \\
52121.15 \\
52155.76 \\
52492.98 \\
52580.43\end{array}$ & $\begin{array}{l}5 \\
3 \\
1 \\
4 \\
3\end{array}$ & $\begin{array}{l}\text { 1. } 102 \\
\text { 1. } 086 \\
\text { 1. } 613 \\
\text { 1. } 101 \\
\text { 1. } 412\end{array}$ & $\begin{array}{l}58572.56 \\
59351.96 \\
59692.16 \\
59838.00 \\
60241.07\end{array}$ & $\begin{array}{l}5 \\
2 \\
5 \\
5 \\
3\end{array}$ & 1. 176 \\
\hline $\begin{array}{l}38270.63 \\
38515.55 \\
38535.21 \\
38962.32 \\
39295.81\end{array}$ & $\begin{array}{l}3 \\
2 \\
1 \\
3 \\
3\end{array}$ & $\begin{array}{l}\text { 1. } 010 \\
1.006 \\
0.472 \\
0.976 \\
1.138\end{array}$ & $\begin{array}{l}46831.35 \\
46850.64 \\
47168.90 \\
47280.89 \\
47514.52\end{array}$ & $\begin{array}{l}3 \\
2 \\
3 \\
4 \\
2\end{array}$ & $\begin{array}{l}\text { 1. } 214 \\
\text { 1. } 110 \\
\text { 1. } 096 \\
\text { 1. } 190 \\
\text { 1. } 284\end{array}$ & $\begin{array}{l}52824.49 \\
52846.28 \\
53010.95 \\
53234.65 \\
53300.59\end{array}$ & $\begin{array}{l}1 \\
6 \\
2 \\
5 \\
2\end{array}$ & $\begin{array}{l}0.907 \\
\text { 1. } 180 \\
\text { 1. } 464 \\
\text { 1. } 146 \\
\text { 1. } 380\end{array}$ & $\begin{array}{l}60325.15 \\
60479.60 \\
60549.32 \\
60870.30 \\
61105.69\end{array}$ & $\begin{array}{l}3 \\
1 \\
5 \\
2 \\
4\end{array}$ & $\begin{array}{l}\text { 1. } 151 \\
0.662 \\
0.904 \\
\text { 1. } 130 \\
\text { 1. } 007\end{array}$ \\
\hline $\begin{array}{l}39743.67 \\
40023.68 \\
40233.46 \\
40304.78 \\
40835.45\end{array}$ & $\begin{array}{l}4 \\
0 \\
2 \\
1 \\
6\end{array}$ & $\begin{array}{l}\text { 1. } 165 \\
\text { 1. } 225\end{array}$ & $\begin{array}{l}47595.98 \\
47620.17 \\
47801.03 \\
47825.41 \\
47829.75\end{array}$ & $\begin{array}{l}1 \\
1 \\
0 \\
3 \\
6\end{array}$ & $\begin{array}{l}\text { 1. } 189 \\
\text { 1. } 300\end{array}$ & $\begin{array}{l}53343.56 \\
53465.79 \\
53644.83 \\
53746.80 \\
54048.81\end{array}$ & $\begin{array}{l}4 \\
3 \\
2 \\
1 \\
5\end{array}$ & $\begin{array}{l}\text { 1. } 071 \\
\text { 1. } 216 \\
\text { 1. } 352 \\
\text { 1. } 234 \\
\text { 1. } 260\end{array}$ & $\begin{array}{l}61357.45 \\
61588.16 \\
61608.62 \\
61694.17 \\
62115.97\end{array}$ & $\begin{array}{l}5 \\
6 \\
6 \\
3 \\
4\end{array}$ & $\begin{array}{l}0.937 \\
1.070\end{array}$ \\
\hline $\begin{array}{l}41144.94 \\
41355.11 \\
41554.42 \\
41709.01 \\
41775.29\end{array}$ & $\begin{array}{l}2 \\
1 \\
3 \\
5 \\
4\end{array}$ & $\begin{array}{l}\text { 1. } 152 \\
\text { 1. } 885 \\
\text { 1. } 207 \\
\text { 1. } 244 \\
\text { 1. } 249\end{array}$ & $\begin{array}{l}47857.60 \\
48064.57 \\
48166.15 \\
48223.08 \\
48470.41\end{array}$ & $\begin{array}{l}5 \\
0 \\
2 \\
2 \\
4\end{array}$ & $\begin{array}{l}\text { 1. } 446 \\
\text { 1. } 276\end{array}$ & $\begin{array}{l}54206.69 \\
54533.78 \\
54648.73 \\
55128.38 \\
55381.25\end{array}$ & $\begin{array}{l}4 \\
2 \\
3 \\
3 \\
4\end{array}$ & $\begin{array}{l}\text { 1. } 157 \\
1.016 \\
0.943 \\
1.260 \\
1.020\end{array}$ & $\begin{array}{l}62204.91 \\
62296.25 \\
62317.92 \\
62463.43 \\
62854.50\end{array}$ & $\begin{array}{l}5 \\
1 \\
2 \\
4 \\
1\end{array}$ & $\begin{array}{l}0.600 \\
0.986 \\
1.167 \\
0.908\end{array}$ \\
\hline $\begin{array}{l}42122.91 \\
42153.29 \\
42959.55 \\
43064.86 \\
43068.72\end{array}$ & $\begin{array}{l}4 \\
2 \\
3 \\
2 \\
0\end{array}$ & $\begin{array}{l}\text { 1. } 258 \\
\text { 1. } 212 \\
\text { 1. } 120 \\
\text { 1. } 041\end{array}$ & $\begin{array}{l}48666.56 \\
48776.29 \\
48962.54 \\
49055.18 \\
49080.51\end{array}$ & $\begin{array}{l}2 \\
1 \\
3 \\
5 \\
2\end{array}$ & $\begin{array}{l}\text { 1. } 131 \\
0.746 \\
\text { 1. } 283 \\
\text { 1. } 212 \\
\text { 1. } 320\end{array}$ & $\begin{array}{l}55505.08 \\
55528.22 \\
55543.11 \\
55551.60 \\
55859.32\end{array}$ & $\begin{array}{l}5 \\
1 \\
3 \\
2 \\
2\end{array}$ & $\begin{array}{l}\text { 1. } 054 \\
\text { 1. } 464 \\
\text { 1. } 430 \\
\text { 1. } 031 \\
\text { 1. } 343\end{array}$ & $\begin{array}{l}63060.46 \\
63485.65 \\
63703.35 \\
63820.47 \\
64653.35\end{array}$ & $\begin{array}{l}2 \\
4 \\
5 \\
4 \\
3\end{array}$ & $\begin{array}{l}0.977 \\
1.01 \\
1.079\end{array}$ \\
\hline $\begin{array}{l}43544.46 \\
43553.67 \\
43700.05 \\
44005.20 \\
44206.24\end{array}$ & $\begin{array}{l}3 \\
1 \\
5 \\
4 \\
1\end{array}$ & $\begin{array}{l}\text { 1. } 147 \\
\text { 1. } 137 \\
\text { 0. } 242\end{array}$ & $\begin{array}{l}49522.55 \\
49536.24 \\
49592.90 \\
49646.62 \\
49886.51\end{array}$ & $\begin{array}{l}5 \\
3 \\
2 \\
3 \\
5\end{array}$ & $\begin{array}{l}0.841 \\
\text { 1. } 317 \\
\text { 1. } 629 \\
1.048\end{array}$ & $\begin{array}{l}55878.70 \\
56018.76 \\
56142.53 \\
56351.09 \\
56450.84\end{array}$ & $\begin{array}{l}1 \\
2 \\
4 \\
3 \\
5\end{array}$ & $\begin{array}{l}\text { 1. } 425 \\
\text { 1. } 002 \\
\text { 1. } 270 \\
\text { 1. } 200 \\
\text { 1. } 045\end{array}$ & $\begin{array}{l}64909.19 \\
65225.24 \\
65495.18 \\
65657.89 \\
66545.40\end{array}$ & $\begin{array}{l}6 \\
4 \\
3 \\
3 \\
4\end{array}$ & 1. 013 \\
\hline $\begin{array}{l}44259.20 \\
44430.39 \\
44434.79 \\
44585.17 \\
44626.00\end{array}$ & $\begin{array}{l}2 \\
3 \\
0 \\
5 \\
4\end{array}$ & $\begin{array}{l}\text { 1. } 297 \\
\text { 1. } 265\end{array}$ & $\begin{array}{l}49886.97 \\
49937.74 \\
50314.43 \\
50507.12 \\
50531.17\end{array}$ & $\begin{array}{l}1 \\
4 \\
3 \\
5 \\
1\end{array}$ & $\begin{array}{l}\text { 2. } 006 \\
\text { 1. } 207 \\
\text { 1. } 281 \\
\text { 1. } 080 \\
\text { 1. } 370\end{array}$ & $\begin{array}{l}56450.95 \\
56521.78 \\
56662.76 \\
56753.05 \\
56987.88\end{array}$ & $\begin{array}{l}3 \\
5 \\
6 \\
4 \\
4\end{array}$ & $\begin{array}{l}\text { 1. } 197 \\
\text { 1. } 118\end{array}$ & $\begin{array}{l}66610.27 \\
66930.35 \\
68213.70 \\
68299.80 \\
70892.62\end{array}$ & $\begin{array}{l}5 \\
1 \\
5 \\
3 \\
2\end{array}$ & 1. 746 \\
\hline 44835. 20 & 3 & 1. 242 & 50693.61 & 6 & & 57060.81 & 3 & 1. 390 & 71240.50 & 5 & 1. 214 \\
\hline
\end{tabular}

\section{References}

[1] C. C. Kiess, J. Research NBS 47, 385 (1951) RP2266; ibid. 51, 247 (1953) RP2457.

[2] C. C. Kiess, J. Research NBS 60, 375 (1958) RP 2856.

[3] H. Kayser, Handbuch der Spectroscopie, S. Hirzel and Co., Leipzig (1912).

[4] G. R. Harrison; Wavelength Tables (John Wiley \& Sons, Inc., New York, N.Y., 1939).

[5] E. McMillan and N. R. Grace, Phys. Rev. 44, 949 (1933).

[6] B. Edlén, J. Opt. Soc. Am. 43, 339 (1953).
[7] C. C. Kiess, G. R. Harrison, and W. J. Hitcheock, J. Research NBS 44, 245 (1950) RP2075.

[8] G. J. van den Berg, Dissertation, University of Amsterdam (1951)

[9] R. E. Trees, W. F. Cahill, and P. Rabinowitz, J. Research NBS 55, 335 (1955) RP2639.

[10] R. E. Trees; Phys. Rev. 123, 1293 (1961).

111] R. E. Trees, Bull. Am. Phys. Soc. 3, 29 (1958).

[12] W. Finkelnburg and W. Humbach, Naturwiss. 42, 35 (1955).

(Paper 66A2-146) 